\title{
Matches and mismatches in the descriptions of semi-inclusive processes at low and high transverse momentum
}

\author{
Alessandro Bacchetta, ${ }^{a *}$ Daniël Boer, ${ }^{b}$ Markus Diehl ${ }^{a}$ and Piet J. Mulders ${ }^{b}$ \\ ${ }^{a}$ Theory Group, Deutsches Elektronen-Synchroton DESY, \\ Notkestr. 85, 22607 Hamburg, Germany \\ ${ }^{b}$ Dept. of Physics and Astronomy, Vrije Universiteit Amsterdam, \\ De Boelelaan 1081, 1081 HV Amsterdam, The Netherlands \\ E-mail: alessandro.bacchetta@jlab.org, dboer@few.vu.nl, \\ markus.diehl@desy.de, mulders@few.vu.nl
}

Abstract: We investigate the transverse-momentum-dependence in semi-inclusive deep inelastic leptoproduction of hadrons. There are two different theoretical approaches to study this dependence, one for low and one for high transverse momentum of the observed hadron. We systematically investigate their connection, paying special attention to azimuthal distributions and to polarization dependence. In the region of intermediate transverse momentum, where both approaches are applicable, we find that their results match for certain observables but not for others. Interpolating expressions are discussed for the case where one has no matching. We then use power counting to determine which mechanism is dominant in various azimuthal and spin asymmetries that are integrated over the transverse momentum. Our findings have consequences for the extension of transversemomentum-dependent factorization beyond leading twist. They also shed light on the problem of resumming logarithms of transverse momentum for azimuthal distributions. Our results can be carried over to the Drell-Yan process and to two-hadron production in $e^{+} e^{-}$annihilation.

KEYwords: Deep Inelastic Scattering, Spin and Polarization Effects, QCD.

\footnotetext{
${ }^{*}$ Present address: Theory Center, Jefferson Lab, 12000 Jefferson Ave., Newport News, VA 23606, U.S.A.
} 


\section{Contents}

1. Introduction 2

2. Structure functions in semi-inclusive deep inelastic scattering 5

3. Factorization and $q_{T}$ resummation

3.1 Collins-Soper factorization 8

3.2 Collins-Soper-Sterman resummation 11

3.3 Azimuthal dependence and polarization 13

4. From high to intermediate $q_{T}$

5. From low to intermediate $q_{T}$ : power counting 20

5.1 Transverse-momentum-dependent distribution and fragmentation functions 20

5.2 Collinear distribution and fragmentation functions 23

5.3 Distribution and fragmentation functions at high transverse momentum 25

5.4 Results for structure functions 33

6. Comparing results at intermediate $q_{T}$

6.1 Interpolating from low to high $q_{T}$

7. Integrating over $q_{T}$

7.1 Behavior of integrated and weighted observables 43

7.2 Polarization dependence 48

8. From low to intermediate $q_{T}$ : explicit calculation 50

8.1 High- $p_{T}$ tails of distribution functions 50

8.2 High- $k_{T}$ tails of fragmentation functions 55

8.3 Results for structure functions and their consequences 57

9. Summary 60

A. Distribution functions at high $p_{T}$ : Feynman versus axial gauge 63

B. Integrated distribution functions and transverse-momentum cutoff 65

G. One-loop expression of the soft factor 66 


\section{Introduction}

Short-distance factorization is a key concept in quantum chromodynamics, providing much of the predictive power of the theory in high-energy scattering processes. Among the simplest processes to which this concept can be applied are one-particle or single jet inclusive production in lepton-nucleon scattering, two-particle or dijet inclusive production in $e^{+} e^{-}$ annihilation, and Drell-Yan lepton pair production via a photon or electroweak gauge boson in hadron-hadron collisions. Crossing of the hard-scattering subprocess closely relates these reactions, and many results obtained for one of them carry over to the other ones. A number of nontrivial issues for factorization arise especially when one observes the transverse momentum $q_{T}$ and the angular distribution of the produced particle with respect to a suitable reference direction. The problem then involves three scales, namely the scale of nonperturbative QCD dynamics, which we represent by the nucleon mass $M$, the transverse momentum $q_{T}$, and the photon or electroweak boson virtuality $Q$, which throughout this paper we require to be large compared with $M$.

There are two basic descriptions for the production of a particle with specified transverse momentum. One of them is applicable for $q_{T} \ll Q$ and involves transversemomentum-dependent (also called unintegrated) parton distribution and fragmentation functions. The other one requires that $q_{T} \gg M$ and generates transverse momentum in the final state by perturbative radiation, using collinear (or integrated) distribution and fragmentation functions as nonperturbative input. In the following we refer to the two momentum regions and the associated theoretical descriptions as "low- $q_{T}$ " and "high- $q_{T}$ ", respectively. The low- and high- $q_{T}$ domains overlap for $M \ll q_{T} \ll Q$, where both descriptions can hence be applied. An important question is whether in this intermediate $q_{T}$ region they describe the same dynamics or two competing mechanisms. Depending on the answer, one can either try to construct a formulation that smoothly interpolates between the two descriptions, or to add their results in a consistent manner.

For the cross section depending on $q_{T}^{2}$ but integrated over the angular distribution of the produced particle, the work of Collins, Soper and Sterman [1] showed that the descriptions based on intrinsic transverse momentum and on hard perturbative radiation indeed match at intermediate $q_{T}$ and permit a smooth interpolation at all orders in $\alpha_{s}$. A key element of the derivation was that for sufficiently large transverse momentum one can express unintegrated parton distributions and fragmentation functions in terms of their integrated counterparts and of perturbatively calculable hard-scattering kernels. The matching of the two descriptions allowed the authors of [1] to resum large logarithms $\ln \left(Q^{2} / q_{T}^{2}\right)$ to all orders using renormalization group techniques - a procedure that remains the cornerstone for transverse-momentum resummation in a wide range of collider processes.

For the angular distribution, however, the situation is less well understood. Both mechanisms just mentioned give rise to nontrivial angular dependence, as has been pointed out long ago for semi-inclusive deep inelastic scattering (SIDIS) by Cahn [2, 3] and by Georgi and Politzer [4 for the low- and high- $q_{T}$ mechanism, respectively. To the best of our knowledge, the relation between the two descriptions for the angular distribution in unpolarized scattering has not been analyzed so far. Important progress has recently 
been made in the understanding of a particular azimuthal asymmetry for a transversely polarized target in SIDIS or Drell-Yan production. The authors of [5-8] have shown that the description of this asymmetry by the Sivers effect for small $q_{T}$ and by the Qiu-Sterman mechanism for large $q_{T}$ match at order $\alpha_{s}$ in the intermediate region $M \ll q_{T} \ll Q$. It is natural to ask if one has a similar situation for other observables as well.

In the present work we therefore present a systematic analysis of the interplay between the low- $q_{T}$ and the high- $q_{T}$ mechanisms for angular distributions, both in unpolarized and in polarized scattering. This provides guidance for the theoretical description of a variety of observables, determining in particular whether or not one should add different contributions. For definiteness we will consider the case of SIDIS, but as remarked above, analogous studies can be performed for $e^{+} e^{-}$collisions and for the Drell-Yan process. Our results are relevant to the possible extension of transverse-momentum resummation for specific azimuthal distributions, which was recently considered for the case of Drell-Yan production in [9] and [10].

A key finding of our work is that for certain observables, the leading terms of the low-and high- $q_{T}$ descriptions match in the region $M \ll q_{T} \ll Q$ of intermediate transverse momenta, whereas for others they do not match. That this may happen can be understood already at the level of power counting. The low- $q_{T}$ description, which uses transverse momentum dependent parton densities and fragmentation functions, is based on taking $Q^{2}$ large compared with $q_{T}^{2}$ and all nonperturbative scales. We chose $q_{T} / Q$ rather than $M / Q$ as parameter for power counting, since in the intermediate- $q_{T}$ region it is the larger of the two. Taking for example an observable $F$ with mass dimension -2 , we can thus expand

$$
F\left(q_{T}, Q\right) \stackrel{q_{T} \lll Q}{=} \frac{1}{M^{2}} \sum_{n}\left[\frac{q_{T}}{Q}\right]^{n-2} l_{n}\left(\frac{M}{q_{T}}\right),
$$

where $l_{n}$ are dimensionless functions. In our applications, the term with index $n$ will correspond to twist- $n$ accuracy in the low- $q_{T}$ calculation, where $n \geq 2$. In the region of intermediate $q_{T}$ we can further expand the functions $l_{n}\left(M / q_{T}\right)$ for small $M / q_{T}$ and then have

$$
F\left(q_{T}, Q\right) \stackrel{M \ll \underline{q_{T}} \ll Q}{\frac{1}{M^{2}}} \sum_{n, k} l_{n, k}\left[\frac{q_{T}}{Q}\right]^{n-2}\left[\frac{M}{q_{T}}\right]^{k}
$$

with coefficients $l_{n, k}$. The high- $q_{T}$ calculation, which is based on collinear factorization, treats both $Q$ and $q_{T}$ as large compared with nonperturbative scales like $M$. The relevant parameter for power counting in the intermediate region is therefore $M / q_{T}$, and we have

$$
F\left(q_{T}, Q\right) \stackrel{M \lll q_{T}}{=} \frac{1}{M^{2}} \sum_{n}\left[\frac{M}{q_{T}}\right]^{n} h_{n}\left(\frac{q_{T}}{Q}\right)
$$

with dimensionless functions $h_{n}$. In our applications, the term with index $n$ will correspond to twist $n$ in the high- $q_{T}$ calculation, where again $n \geq 2$. In the intermediate- $q_{T}$ region we can then expand $h_{n}\left(q_{T} / Q\right)$ for small $q_{T} / Q$ :

$$
F\left(q_{T}, Q\right) \stackrel{M \ll q_{T} \ll Q}{=} \frac{1}{M^{2}} \sum_{n, k} h_{n, k}\left[\frac{M}{q_{T}}\right]^{n}\left[\frac{q_{T}}{Q}\right]^{k-2}
$$


with coefficients $h_{n, k}$. Since both (1.2) and (1.4) are valid in the intermediate region, we can identify the coefficients $l_{n, k}=h_{k, n}$. As a consequence, a term of twist $n$ in the low- $q_{T}$ calculation will only correspond to a term of the same twist in the high- $q_{T}$ calculation if $n=k$. We will for instance encounter observables with

$$
M^{2} F\left(q_{T}, Q\right)=l_{2,2}\left[\frac{q_{T}}{Q}\right]^{0}\left[\frac{M}{q_{T}}\right]^{2}+l_{4,2}\left[\frac{q_{T}}{Q}\right]^{2}\left[\frac{M}{q_{T}}\right]^{2}+\cdots,
$$

where the term with $l_{2,2}=h_{2,2}$ is of leading twist in both the low- and high- $q_{T}$ calculations. The term with $l_{4,2}=h_{2,4}$ is subleading in the low- $q_{T}$ calculation and becomes subleading in the high- $q_{T}$ when one takes the additional limit $q_{T} \ll Q$. For other observables, we will find

$$
M^{2} F\left(q_{T}, Q\right)=l_{2,4}\left[\frac{q_{T}}{Q}\right]^{0}\left[\frac{M}{q_{T}}\right]^{4}+l_{4,2}\left[\frac{q_{T}}{Q}\right]^{2}\left[\frac{M}{q_{T}}\right]^{2}+\cdots .
$$

Here the term with $l_{2,4}=h_{4,2}$ is leading in the low- $q_{T}$ calculation but subleading in the high- $q_{T}$ one, whereas the reverse holds for the term with $l_{4,2}=h_{2,4}$. The respective leadingorder terms in the two calculations will hence not match in the intermediate region of $q_{T}$. Which term in (1.6) is larger in given kinematics obviously depends on the relative size of the two small parameters $q_{T} / Q$ and $M / q_{T}$. We will discuss in section 6.1 how one can construct interpolating expressions using both terms.

An important question is which terms in the expansions (1.1) and (1.3), and hence in (1.2) and (1.4), can actually be calculated in practice. We discuss this in some detail in the main body of the paper, but already mention here that in the high- $q_{T}$ framework there is a large number of results at twist two and three. In low- $q_{T}$ framework factorization is rather well understood at twist-two level, whereas its status is less clear at twist three. Little is known about the validity of factorization at twist-four accuracy in either framework. In the example (1.6) one can thus envisage to compute the terms $l_{2,4}$ and $h_{2,4}$, which are leading in their respective power counting scheme. The simultaneous validity of the expansions (1.2) and (1.4) in the intermediate region requires that $h_{4,2}=l_{2,4}$ and $l_{4,2}=h_{2,4}$, but at present one cannot check this explicitly because a calculation of the power-suppressed terms $h_{4,2}$ and $l_{4,2}$ is beyond the state of the art.

Several investigations have been performed assuming factorization for twist-three observables in the low- $q_{T}$ description. Detailed calculations at tree level 11-14 are found to be self-consistent and give results with a structure similar to that of twist-two observables. Their extension to higher orders in $\alpha_{s}$, including a proper treatment of soft gluon exchange has not been achieved yet, and the study [15] suggests that such an extension will not be trivial. In sections 5.4 and 8.3 we will investigate observables where the leading terms in the expansions (1.2) and (1.4) coincide and have the coefficient $l_{3,2}=h_{2,3}$. The twist-two quantity $h_{2,3}$ is readily computed, and its comparison with the result for $l_{3,2}$ obtained with a candidate factorization formula will shed light on low- $q_{T}$ factorization at twist three.

In experimental analyses one often has to integrate over the observed $q_{T}$ in order to accumulate statistics. One may simply integrate an observable over $q_{T}^{2}$ or consider weighted observables like $\int d q_{T}^{2}\left(q_{T} / M\right)^{p} F\left(q_{T}, Q\right)$ with some power $p$. If in turn the measurement of the $q_{T}$-dependence suffers from large uncertainties, then both a differential 
observable and weighted integrals will be affected with large errors, so that the simple integral $\int d q_{T}^{2} F\left(q_{T}, Q\right)$ may be the best quantity to consider from an experimental point of view. For the theoretical analysis it is important to identify the relative importance of the different $q_{T}$ regions in an integrated observable, and to clarify their interplay if several regions are important. Our results will allow us to address this question at the level of power counting.

Our paper is organized as follows. In the next section we define the structure functions for SIDIS, which are the observables we study in detail in this work. To set the stage, we recall in section 3 some important results for SIDIS taken differential in $q_{T}$ but integrated over the angular distribution of the observed hadron, recalling in particular the foundations of $q_{T}$ resummation in this context. In section 1 we collect the well-known results of the calculation of SIDIS with $q_{T} \gg M$ in collinear factorization at leading order in $\alpha_{s}$, and then approximate these results for $q_{T} \ll Q$. In section 5 we take the opposite path, recalling the results for SIDIS with $q_{T} \ll Q$ and approximating them for $q_{T} \gg M$. For this we need the behavior of distribution and fragmentation functions at high transverse momentum, and we will derive the corresponding power behavior of these functions based on general grounds. In section 6 we will see for which observables the calculations of the two previous sections match for intermediate $q_{T}$ and for which ones they do not. The consequences for integrated observables are discussed in section 0 . Whereas in section 5 we derive the power behavior for all structure functions introduced in section 2, we give in section 8 explicit results for those observables that appear at twist two in the high- $q_{T}$ regime. The comparison of the high- $q_{T}$ with the low- $q_{T}$ expressions will allow us to draw some conclusions about the unsolved problem of $q_{T}$ resummation for angular distributions, as well as the possibility of extending low- $q_{T}$ factorization to twist three. The main results of our work are summarized in section 9, and some technical details are given in the appendices.

\section{Structure functions in semi-inclusive deep inelastic scattering}

The physical process we investigate in this work is semi-inclusive DIS,

$$
\ell(l)+p(P) \rightarrow \ell\left(l^{\prime}\right)+h\left(P_{h}\right)+X,
$$

where $\ell$ denotes the beam lepton, $p$ the proton target, and $h$ the observed hadron, with four-momenta given in parentheses. We allow for polarization of beam and target, but restrict ourselves to the case of an unpolarized final state, i.e. to the situation in which $h$ has spin zero or where its polarization is not observed. The corresponding observables cover a variety of situations with different types of power behavior we wish to discuss. Many of them have been measured in experiment, see [16-21] and the recent review in 22].

Working in the one-photon exchange approximation, we define the photon momentum $q=l-l^{\prime}$ and its virtuality $Q^{2}=-q^{2}$. We use the conventional variables for SIDIS

$$
x=\frac{Q^{2}}{2 P \cdot q}, \quad \quad y=\frac{P \cdot q}{P \cdot l}, \quad z=\frac{P \cdot P_{h}}{P \cdot q},
$$




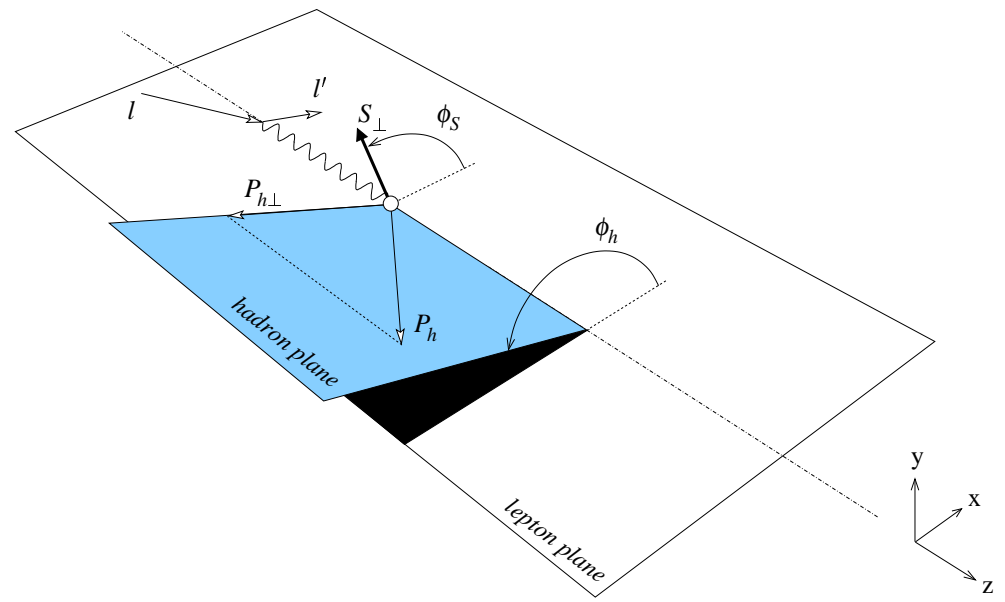

Figure 1: Definition of azimuthal angles for semi-inclusive deep inelastic scattering in the target rest frame [23]. $P_{h \perp}$ and $S_{\perp}$ are the transverse parts of $P_{h}$ and $S$ with respect to the photon momentum.

and write $M$ and $M_{h}$ for the respective masses of the proton target and the produced hadron $h$. We take the limit of large $Q^{2}$ at fixed $x, y, z$, and throughout this paper we neglect corrections in the masses of the hadrons or the lepton.

It is convenient to discuss the experimental observables for SIDIS in a frame where $P$ and $q$ collinear. We define the transverse part $P_{h \perp}^{\mu}$ of $P_{h}^{\mu}$ as orthogonal with respect to the momenta $P$ and $q$. Likewise, we define the transverse part $S_{\perp}^{\mu}$ of the spin vector $S^{\mu}$ of the target, as well as its longitudinal projection $S_{\|}$along $P^{\mu}$. We further define the azimuthal angles $\phi_{h}$ and $\phi_{S}$ of $P_{h}^{\mu}$ and $S^{\mu}$ with respect to the lepton plane in accordance with the Trento conventions [23], as shown in figure 1. Covariant expressions for the quantities just discussed can be found in [14. Finally, we write $\lambda_{e}$ for the longitudinal polarization of the incoming lepton, with $\lambda_{e}=1$ corresponding to a purely right-handed beam.

The lepton-hadron cross section can then be parameterized as 14

$$
\begin{aligned}
& \frac{d \sigma}{d x d y d z d \phi_{S} d \phi_{h} d P_{h \perp}^{2}}=\frac{\alpha^{2}}{x Q^{2}} \frac{y}{2(1-\varepsilon)} \\
& \times\left\{F_{U U, T}+\varepsilon F_{U U, L}+\sqrt{2 \varepsilon(1+\varepsilon)} \cos \phi_{h} F_{U U}^{\cos \phi_{h}}+\varepsilon \cos \left(2 \phi_{h}\right) F_{U U}^{\cos 2 \phi_{h}}\right. \\
& +\lambda_{e} \sqrt{2 \varepsilon(1-\varepsilon)} \sin \phi_{h} F_{L U}^{\sin \phi_{h}} \\
& +S_{\|}\left[\sqrt{2 \varepsilon(1+\varepsilon)} \sin \phi_{h} F_{U L}^{\sin \phi_{h}}+\varepsilon \sin \left(2 \phi_{h}\right) F_{U L}^{\sin 2 \phi_{h}}\right] \\
& +S_{\|} \lambda_{e}\left[\sqrt{1-\varepsilon^{2}} F_{L L}+\sqrt{2 \varepsilon(1-\varepsilon)} \cos \phi_{h} F_{L L}^{\cos \phi_{h}}\right] \\
& +\left|\boldsymbol{S}_{\perp}\right|\left[\sin \left(\phi_{h}-\phi_{S}\right)\left(F_{U T, T}^{\sin \left(\phi_{h}-\phi_{S}\right)}+\varepsilon F_{U T, L}^{\sin \left(\phi_{h}-\phi_{S}\right)}\right)\right. \\
& +\varepsilon \sin \left(\phi_{h}+\phi_{S}\right) F_{U T}^{\sin \left(\phi_{h}+\phi_{S}\right)}+\varepsilon \sin \left(3 \phi_{h}-\phi_{S}\right) F_{U T}^{\sin \left(3 \phi_{h}-\phi_{S}\right)}
\end{aligned}
$$




$$
\begin{gathered}
\left.+\sqrt{2 \varepsilon(1+\varepsilon)} \sin \phi_{S} F_{U T}^{\sin \phi_{S}}+\sqrt{2 \varepsilon(1+\varepsilon)} \sin \left(2 \phi_{h}-\phi_{S}\right) F_{U T}^{\sin \left(2 \phi_{h}-\phi_{S}\right)}\right] \\
+\left|\boldsymbol{S}_{\perp}\right| \lambda_{e}\left[\sqrt{1-\varepsilon^{2}} \cos \left(\phi_{h}-\phi_{S}\right) F_{L T}^{\cos \left(\phi_{h}-\phi_{S}\right)}+\sqrt{2 \varepsilon(1-\varepsilon)} \cos \phi_{S} F_{L T}^{\cos \phi_{S}}\right. \\
\left.\left.+\sqrt{2 \varepsilon(1-\varepsilon)} \cos \left(2 \phi_{h}-\phi_{S}\right) F_{L T}^{\cos \left(2 \phi_{h}-\phi_{S}\right)}\right]\right\}
\end{gathered}
$$

where $\alpha$ is the fine structure constant and $\varepsilon$ the ratio of longitudinal and transverse photon flux,

$$
\varepsilon=\frac{1-y}{1-y+y^{2} / 2} .
$$

The 18 structure functions $F$ on the r.h.s. depend on $x, Q^{2}, z$ and $P_{h \perp}^{2}$ and encode the strong-interaction dynamics of the hadronic subprocess $\gamma^{*}+p \rightarrow h+X$. Their first and second subscript respectively specifies the polarization of the beam and the target. In the structure functions $F_{U U, T}, F_{U U, L}$ and $F_{U T, T}^{\sin \left(\phi_{h}-\phi_{S}\right)}, F_{U T, L}^{\sin \left(\phi_{h}-\phi_{S}\right)}$, the third subscript refers to the transverse and longitudinal polarization of the photon.

To calculate the SIDIS structure functions it is convenient to use light-cone coordinates with respect to the directions of the relevant hadron momenta. We introduce light-like vectors $n_{+}$and $n_{-}$with $n_{+} \cdot n_{-}=1$ such that, up to mass corrections, $n_{+}$is proportional to $P$ and $n_{-}$proportional to $P_{h}$. A rescaling

$$
n_{+} \rightarrow \kappa n_{+}, \quad n_{-} \rightarrow \kappa^{-1} n_{-}
$$

corresponds to boosts in the collinear direction. The off-collinearity of the process is determined by the vector

$$
q_{T}^{\mu}=q^{\mu}+(1-r) x P^{\mu}-P_{h}^{\mu} / z
$$

with $r=q_{T}^{2} / Q^{2}$. For ease of notation we denote the length of this vector by

$$
q_{T}=\left(-q_{T}^{\mu} q_{T \mu}\right)^{1 / 2},
$$

so that $q_{T}^{2}$ is positive. There is a simple relation between the transverse momentum $q_{T}^{\mu}$ of the photon with respect to the hadrons and the transverse momentum $P_{h \perp}^{\mu}$ of the produced hadron with respect to the photon and proton: $P_{h \perp}^{\mu}=-z q_{T}^{\mu}-2 r z x P^{\mu}$. The SIDIS cross section differential in $q_{T}^{2}$ instead of $P_{h \perp}^{2}$ is hence equal to $z^{2}$ times the r.h.s. of (2.3).

\section{Factorization and $q_{T}$ resummation}

In this section we recall some important results for the description of hard processes with measured $q_{T}$, in particular the factorization for low $q_{T}$ formulated by Collins and Soper 24] and its connection to the procedure of transverse momentum resummation by Collins, Soper and Sterman [1]. In the following we refer to these authors as CS and CSS, respectively. In the next two subsections we focus on the unpolarized SIDIS cross section differential in $q_{T}$ but integrated over the azimuthal angle $\phi_{h}$. 


\subsection{Collins-Soper factorization}

In the work of CS, factorization was derived for the production of back-to-back jets in electron-positron annihilation, or more specifically for $e^{+} e^{-} \rightarrow A+B+X$, where $A$ and $B$ are two hadrons belonging to opposite-side jets in the $e^{+} e^{-}$c.m. In general the momenta $P_{A}$ and $P_{B}$ of the two hadrons are not exactly back-to-back because of their recoil against the additional particles $X$ produced in the process. The cross section, or equivalently the hadron tensor, depends on the transverse momentum $q_{T}^{\mu}$ of the virtual photon w.r.t. the hadrons in the c.m. of $A$ and $B$, which is the analog of $q_{T}^{\mu}$ introduced for SIDIS in (2.6). For $q_{T} \ll Q$ the CS paper derived a factorized expression of the hadron tensor, which is a convolution in transverse momentum of a soft factor $U$ and two fragmentation functions $D_{1}^{A / a}$ and $D_{1}^{B / \bar{a}}$ for the fragmentation of a quark or antiquark into $A$ or $B$. In addition there is a hard-scattering factor $H$, which does not depend on any transverse momentum. To be specific, eq. (7.14) in [24] gives the following expression for the hadron tensor:

$$
\begin{aligned}
W_{e^{+} e^{-}}^{\mu \nu} \propto & \operatorname{Tr}\left\{\not P_{A} \gamma^{\mu} \not P_{B} \gamma^{\nu}\right\}\left|H_{e^{+} e^{-}}\left(z_{A}^{-1} \zeta_{A}^{1 / 2}, z_{B}^{-1} \zeta_{B}^{1 / 2}\right)\right|^{2} \sum_{a} e_{a}^{2} \int d^{2} \boldsymbol{p}_{T} d^{2} \boldsymbol{k}_{T} d^{2} \boldsymbol{l}_{T} \\
& \times \delta^{(2)}\left(\boldsymbol{p}_{T}+\boldsymbol{k}_{T}+\boldsymbol{l}_{T}-\boldsymbol{q}_{T}\right) D_{1}^{A / a}\left(z_{A}, p_{T}^{2} ; \zeta_{A}\right) D_{1}^{B / \bar{a}}\left(z_{B}, k_{T}^{2} ; \zeta_{B}\right) U\left(l_{T}^{2}\right)+\cdots,
\end{aligned}
$$

where ... stands for terms that either vanish after integration over the azimuthal angle of $\boldsymbol{q}_{T}$ or are power suppressed in $1 / Q$. The index $a$ runs over flavors of quarks and of antiquarks with fractional charge $e_{a}$. For consistency within the present paper we have slightly changed notation compared with CS. ${ }^{1}$ The individual factors $H, D_{1}^{A / a}, D_{1}^{B / \bar{a}}$, and $U$ depend on an ultraviolet factorization scale $\mu$, which we have not displayed for brevity. The derivation by $\mathrm{CS}$ is done in an axial gauge specified by a spacelike vector $v$, and the dependence of individual factors on this vector is through the parameters $\zeta_{A}=-\left(2 P_{A} \cdot v\right)^{2} / v^{2}$ and $\zeta_{B}=-\left(2 P_{B} \cdot v\right)^{2} / v^{2}$. More generally, $\zeta_{A}$ and $\zeta_{B}$ serve as cut-offs for rapidity divergences and in a gauge-invariant definition of the fragmentation functions arise from path-ordered exponentials involving the vector $v$, see e.g. 25-27. The hadron tensor as a whole is of course independent of $\zeta_{A}$ and $\zeta_{B}$, so that the dependence on these parameters has to cancel between the fragmentation functions and the hard-scattering factor. The fact that the soft factor defined by CS does not depend on them is less obvious. ${ }^{2}$

In this work we will assume that the factorization of Collins and Soper also holds for the hadron tensor in SIDIS at low $q_{T}$. Such an expression, albeit with some differences, has been obtained by Ji, Ma and Yuan [26], and another relevant investigation has recently been made by Collins, Rogers and Staśto [27], which gives us confidence that our assumption

\footnotetext{
${ }^{1}$ We write $D_{1}$ instead of $\mathcal{P}$ for the fragmentation functions, $v$ instead of $n$ for the gauge fixing vector, and $n_{+}, n_{-}$instead of $v_{A}, v_{B}$ for the light-cone directions. Our normalization condition for $U$ differs from the one of CS by a factor $(2 \pi)^{2}$.

${ }^{2}$ The four-vectors entering the construction of $U$ are $v, n_{+}, n_{-}$and $l_{T}$. The gauge vector $v$ used by CS has a zero transverse component, so that the only scalar products involving $v$ are $v \cdot n_{+}, v \cdot n_{-}$and $v^{2}=2\left(v \cdot n_{+}\right)\left(v \cdot n_{-}\right)$. Gauges related by scaling $v \rightarrow \kappa v$ are equivalent, which leaves only a possible dependence on $v \cdot n_{+} / v \cdot n_{-}$. This is however excluded by boost invariance, which requires that $U$ must not change under the rescaling 2.5$)$.
} 
can be justified. The analog of (3.1) then reads

$$
\begin{aligned}
W_{\text {SIDIS }}^{\mu \nu} \propto & \operatorname{Tr}\left\{\not p \gamma^{\mu} \not p_{h} \gamma^{\nu}\right\}\left|H_{\text {SIDIS }}\left(x \zeta^{1 / 2}, z^{-1} \zeta_{h}^{1 / 2}\right)\right|^{2} \sum_{a} e_{a}^{2} \int d^{2} \boldsymbol{p}_{T} d^{2} \boldsymbol{k}_{T} d^{2} \boldsymbol{l}_{T} \\
& \times \delta^{(2)}\left(\boldsymbol{p}_{T}-\boldsymbol{k}_{T}+\boldsymbol{l}_{T}+\boldsymbol{q}_{T}\right) f_{1}^{a}\left(x, p_{T}^{2} ; \zeta\right) D_{1}^{a}\left(z, k_{T}^{2} ; \zeta_{h}\right) U\left(l_{T}^{2}\right)+\cdots
\end{aligned}
$$

where one of the fragmentation functions has been replaced by the distribution function $f_{1}^{a}$ for quarks or antiquarks in the target. In the following we refer to the factorization expressed in (3.2) as "CS factorization". The result (3.2) gives rise to just one structure function,

$$
\begin{aligned}
F_{U U, T}= & \left|H\left(x \zeta^{1 / 2}, z^{-1} \zeta_{h}^{1 / 2}\right)\right|^{2} \sum_{a} x e_{a}^{2} \int d^{2} \boldsymbol{p}_{T} d^{2} \boldsymbol{k}_{T} d^{2} \boldsymbol{l}_{T} \\
& \times \delta^{(2)}\left(\boldsymbol{p}_{T}-\boldsymbol{k}_{T}+\boldsymbol{l}_{T}+\boldsymbol{q}_{T}\right) f_{1}^{a}\left(x, p_{T}^{2} ; \zeta\right) D_{1}^{a}\left(z, k_{T}^{2} ; \zeta_{h}\right) U\left(l_{T}^{2}\right),
\end{aligned}
$$

where we recall that $H, f_{1}, D_{1}$, and $U$ depend on a renormalization scale $\mu$. For brevity we omit the subscript "SIDIS" in $H$ from now on.

In the intermediate region $M \ll q_{T} \ll Q$, one can go further since at least one of the momenta $\boldsymbol{p}_{T}, \boldsymbol{k}_{T}, \boldsymbol{l}_{T}$ in (3.3) is of order $\boldsymbol{q}_{T}$ and hence large compared with the nonperturbative scale $M$. For large transverse momentum the soft factor $U\left(l_{T}^{2}\right)$ can be calculated order by order in $\alpha_{s}$, whereas $f_{1}^{a}\left(x, p_{T}^{2} ; \zeta\right)$ and $D_{1}^{a}\left(z, k_{T}^{2} ; \zeta_{h}\right)$ are respectively given as convolutions of perturbatively calculable kernels with the collinear distribution and fragmentation functions $f_{1}^{a}(x)$ and $D_{1}^{a}(z)$. We will follow this path in sections 5 and 8 . The accuracy of this procedure is however limited: up to mass corrections one has $\left(\zeta \zeta_{h}\right)^{1 / 2}=x^{-1} z Q^{2}$, and the power counting in the CS derivation requires both $\zeta$ and $\zeta_{h}$ to be of order $Q^{2}$, so that the perturbative expressions for $f_{1}^{a}\left(x, p_{T}^{2} ; \zeta\right)$ and $D_{1}^{a}\left(z, k_{T}^{2} ; \zeta_{h}\right)$ involve large logarithms $\ln \left(Q / q_{T}\right)$. Let us sketch how these logarithms are resummed in the work of CS. The variation of $D_{1}$ with $\zeta_{h}$ is described by the Collins-Soper equation, which gives $\partial D_{1} / \partial \ln \zeta_{h}$ as a convolution in transverse momentum of an evolution kernel with $D_{1}$. Analogous considerations apply to the distribution function $f_{1}^{a}\left(x, p_{T}^{2} ; \zeta\right)$. For the Fourier transformed functions

$$
\begin{aligned}
\tilde{f}_{1}^{a}\left(x, b^{2} ; \zeta\right) & =\int d^{2} \boldsymbol{p}_{T} e^{i \boldsymbol{b} \cdot \boldsymbol{p}_{T}} f_{1}^{a}\left(x, p_{T}^{2} ; \zeta\right) \\
\widetilde{D}_{1}^{a}\left(z, b^{2} ; \zeta_{h}\right) & =\int d^{2} \boldsymbol{k}_{T} e^{i \boldsymbol{b} \cdot \boldsymbol{k}_{T}} D_{1}^{a}\left(z, k_{T}^{2} ; \zeta_{h}\right)
\end{aligned}
$$

one obtains ordinary differential equations, whose solutions can be written as

$$
\begin{aligned}
\tilde{f}_{1}^{a}\left(x, b^{2} ; \zeta\right) & =\hat{f}_{1}^{a}\left(x, b^{2}\right) \exp \left[-\widehat{S}^{\prime}\left(x \zeta^{1 / 2}, b\right)\right], \\
\widetilde{D}_{1}^{a}\left(z, b^{2} ; \zeta_{h}\right) & =\widehat{D}_{1}^{a}\left(z, b^{2}\right) \exp \left[-\widehat{S}^{\prime}\left(z^{-1} \zeta_{h}^{1 / 2}, b\right)\right],
\end{aligned}
$$

where the Sudakov factor $\widehat{S}$ is constructed from the evolution kernel. The structure function in (3.3) can then be rewritten as

$$
\begin{aligned}
F_{U U, T}= & |H(Q, Q ; \mu)|^{2} \sum_{a} x e_{a}^{2} \int \frac{d^{2} \boldsymbol{b}}{(2 \pi)^{2}} e^{-i \boldsymbol{b} \cdot \boldsymbol{q}_{T}} \exp \left[-2 \widehat{S}^{\prime}(Q, b)\right] \widetilde{U}\left(b^{2} ; \mu\right) \\
& \times \hat{f}_{1}^{a}\left(x, b^{2} ; \mu\right) \widehat{D}_{1}^{a}\left(z, b^{2} ; \mu\right)
\end{aligned}
$$


with

$$
\widetilde{U}\left(b^{2} ; \mu\right)=\int d^{2} \boldsymbol{l}_{T} e^{i \boldsymbol{b} \cdot \boldsymbol{l}_{T}} U\left(l_{T}^{2} ; \mu\right) .
$$

Here we have fixed the gauge parameters as $x^{2} \zeta=z^{-2} \zeta_{h}=Q^{2}$ and restored the dependence on the renormalization scale $\mu$. The Sudakov factor $\widehat{S}^{\prime}$ resums large logarithms of $Q b$, which corresponds to large logarithms of $Q / q_{T}$ in $F_{U U, T}$ since the typical values of $b$ in the integral (3.6) are of order $1 / q_{T}$.

For $b \ll 1 / M$ the factors $\widehat{S}^{\prime}, \widetilde{U}, \hat{f}_{1}^{a}$ and $\widehat{D}_{1}^{a}$ can be expanded in perturbation theory. To avoid large logarithms of $\mu b$ in this expansion, one should take the renormalization scale of order $1 / b$. A common choice in the $\overline{\mathrm{MS}}$ scheme is $\mu=b_{0} / b$ with $b_{0}=2 e^{-\gamma_{E}} \approx 1.1$, where $\gamma_{E}$ is the Euler constant. This simplifies a number of perturbative coefficients: in particular the $O\left(\alpha_{s}\right)$ term in the soft factor is then zero, and one has $\widetilde{U}\left(b^{2}, \mu=b_{0} / b\right)=1+O\left(\alpha_{s}^{2}\right)$ up to power corrections in $M b$. The small- $b$ expansion for the distribution and fragmentation functions reads

$$
\begin{aligned}
\hat{f}_{1}^{a}\left(x, b^{2} ; \mu=b_{0} / b\right) & =\sum_{i}\left(\widehat{C}_{a i}^{\text {in }} \otimes f_{1}^{i}\right)\left(x ; \mu=b_{0} / b\right), \\
z^{2} \widehat{D}_{1}^{a}\left(z, b^{2} ; \mu=b_{0} / b\right) & =\sum_{j}\left(D_{1}^{j} \otimes \widehat{C}_{j a}^{\text {out }}\right)\left(z ; \mu=b_{0} / b\right),
\end{aligned}
$$

where the indices $i$ and $j$ run over quarks, antiquarks and the gluon. $f_{1}^{i}(x ; \mu)$ and $D_{1}^{j}(z ; \mu)$ are the usual collinear distribution and fragmentation functions, and $\otimes$ denotes the familiar convolution in longitudinal momentum fractions,

$$
\begin{aligned}
& (C \otimes f)(x ; \mu)=\int_{x}^{1} \frac{d \hat{x}}{\hat{x}} C(\hat{x} ; \mu) f\left(\frac{x}{\hat{x}} ; \mu\right), \\
& (D \otimes C)(z ; \mu)=\int_{z}^{1} \frac{d \hat{z}}{\hat{z}} D\left(\frac{z}{\hat{z}} ; \mu\right) C(\hat{z} ; \mu) .
\end{aligned}
$$

With the scale choice $\mu=b_{0} / b$ we find large logarithms of $Q b$ in $|H|^{2}=1+O\left(\alpha_{s}\right)$. These can readily be resummed using the renormalization group equation for this factor, which allows one to write $\left|H\left(Q, Q ; \mu=b_{0} / b\right)\right|^{2}=|H(Q, Q ; \mu=Q)|^{2} e^{-\widehat{R}(Q, b)}$. In the intermediate region $M \ll q_{T} \ll Q$ one therefore has

$$
\begin{aligned}
F_{U U, T}= & |H(\mu=Q)|^{2} \frac{1}{z^{2}} \sum_{a} x e_{a}^{2} \int \frac{d^{2} \boldsymbol{b}}{(2 \pi)^{2}} e^{-i \boldsymbol{b} \cdot \boldsymbol{q}_{T}} \exp [-\widehat{S}(Q, b)] \widetilde{U}\left(\mu=b_{0} / b\right) \\
& \times \sum_{i}\left(\widehat{C}_{a i}^{\mathrm{in}} \otimes f_{1}^{i}\right)\left(x ; \mu=b_{0} / b\right) \sum_{j}\left(D_{1}^{j} \otimes \widehat{C}_{j a}^{\text {out }}\right)\left(z ; \mu=b_{0} / b\right)
\end{aligned}
$$

with $\widehat{S}=2 \widehat{S}^{\prime}+\widehat{R}$. Here we have used that for dimensional reasons $H(Q, Q ; \mu)$ depends on $Q$ only in the combination $Q / \mu$, so that the only $Q$-dependence in $H(Q, Q ; \mu=Q)$ is through the argument of the running coupling. An analogous statement holds for the $b$ dependence in $\widetilde{U}\left(b^{2} ; \mu=b_{0} / b\right)$ at small $b$. The result (3.10) only involves the usual collinear distribution and fragmentation functions, together with factors $H, \widehat{S}, \widetilde{U}, \widehat{C}^{\text {in }}, \widehat{C}^{\text {out }}$ whose perturbative expansions are free of large logarithms. 


\subsection{Collins-Soper-Sterman resummation}

We now turn to the region of large $q_{T} \gg M$, where one can evaluate the hadron tensor in standard collinear factorization. To leading order in $\alpha_{s}$ we have

$$
\begin{aligned}
& F_{U U, T}=\frac{1}{Q^{2}} \frac{\alpha_{s}}{(2 \pi z)^{2}} \sum_{a} x e_{a}^{2} \int_{x}^{1} \frac{d \hat{x}}{\hat{x}} \int_{z}^{1} \frac{d \hat{z}}{\hat{z}} \delta\left(\frac{q_{T}^{2}}{Q^{2}}-\frac{(1-\hat{x})(1-\hat{z})}{\hat{x} \hat{z}}\right) \\
& \times\left[f_{1}^{a}\left(\frac{x}{\hat{x}}\right) D_{1}^{a}\left(\frac{z}{\hat{z}}\right) C_{U U, T}^{\left(\gamma^{*} q \rightarrow q g\right)}+f_{1}^{a}\left(\frac{x}{\hat{x}}\right) D_{1}^{g}\left(\frac{z}{\hat{z}}\right) C_{U U, T}^{\left(\gamma^{*} q \rightarrow g q\right)}+f_{1}^{g}\left(\frac{x}{\hat{x}}\right) D_{1}^{a}\left(\frac{z}{\hat{z}}\right) C_{U U, T}^{\left(\gamma^{*} g \rightarrow q \bar{q}\right)}\right]
\end{aligned}
$$

with power corrections in $M / q_{T}$. The hard-scattering coefficients $C_{U U, T}$ for the indicated partonic subprocesses are functions of $\hat{x}, \hat{z}$, and $q_{T} / Q$, and will be given in section 4 . Approximating (3.11) for $q_{T} \ll Q$ one obtains

$$
\begin{gathered}
F_{U U, T}=\frac{1}{q_{T}^{2}} \frac{\alpha_{s}}{2 \pi^{2} z^{2}} \sum_{a} x e_{a}^{2}\left[f_{1}^{a}(x) D_{1}^{a}(z) L\left(\frac{Q^{2}}{q_{T}^{2}}\right)+f_{1}^{a}(x)\left(D_{1}^{a} \otimes P_{q q}+D_{1}^{g} \otimes P_{g q}\right)(z)\right. \\
\left.+\left(P_{q q} \otimes f_{1}^{a}+P_{q g} \otimes f_{1}^{g}\right)(x) D_{1}^{a}(z)\right]
\end{gathered}
$$

with power corrections in $q_{T} / Q$ and in $M / q_{T}$. The factor $L$ is defined as

$$
L\left(\frac{Q^{2}}{q_{T}^{2}}\right)=2 C_{F} \ln \frac{Q^{2}}{q_{T}^{2}}-3 C_{F},
$$

and $P_{q q}, P_{g q}, P_{q g}$ are the DGLAP splitting functions at lowest order in $\alpha_{s}$, given in 4.29) below. We see that a large logarithm of $Q^{2} / q_{T}^{2}$ appears in the fixed-order calculation when $q_{T} \ll Q$. Corresponding logarithms at higher orders in $\alpha_{s}$ spoil the convergence of the perturbative series. Collins, Soper and Sterman [1] have shown that these logarithms exponentiate and that their resummation results in a factorized expression, which we will refer to as "CSS factorization". The discussion in the CSS paper is given for the cross section of Drell-Yan production differential in $q_{T}^{2}$ but integrated over the azimuthal angle of $\boldsymbol{q}_{T}$. The corresponding result for SIDIS is given in [28] and can be written as

$$
\begin{aligned}
F_{U U, T}= & \frac{1}{z^{2}} \sum_{a} x e_{a}^{2} \int \frac{d^{2} \boldsymbol{b}}{(2 \pi)^{2}} e^{-i \boldsymbol{b} \cdot \boldsymbol{q}_{T}} \exp [-S(Q, b)] \\
& \times \sum_{i}\left(C_{a i}^{\text {in }} \otimes f_{1}^{i}\right)\left(x ; \mu=b_{0} / b\right) \sum_{j}\left(D_{1}^{j} \otimes C_{j a}^{\text {out }}\right)\left(z ; \mu=b_{0} / b\right) .
\end{aligned}
$$

This form is valid for $M \ll q_{T} \ll Q$. It can be extended to the full large- $q_{T}$ region, $q_{T} \gg M$, by adding the difference between (3.11) and its approximated form (3.12). For further discussion of this matching of resummed and fixed-order terms we refer to [29]. As an aside, we remark that at $q_{T} \sim Q$ the longitudinal structure function $F_{U U, L}$ is parametrically of the same order as $F_{U U, T}$, whereas at $q_{T} \ll Q$ it is suppressed by a relative factor $q_{T}^{2} / Q^{2}$. One may hence also apply the CSS prescription to $F_{U U, T}+\varepsilon F_{U U, L}$ or to $F_{U U, T}+F_{U U, L}$ instead of $F_{U U, T}$. The term to which resummation is applied is the same in all cases, and only the unresummed part of the fixed-order calculation is different. 
Let us see how (3.14) reduces to (3.12) at leading order in $\alpha_{s}$. The Sudakov factor $S(Q, b)$ reads

$$
S(Q, b)=\int_{b_{0}^{2} / b^{2}}^{Q^{2}} \frac{d \mu^{2}}{\mu^{2}}\left[A\left(\alpha_{s}(\mu)\right) \ln \frac{Q^{2}}{\mu^{2}}+B\left(\alpha_{s}(\mu)\right)\right]
$$

with

$$
A\left(\alpha_{s}\right)=\sum_{k=1}^{\infty} A_{k}\left(\frac{\alpha_{s}}{\pi}\right)^{k}, \quad B\left(\alpha_{s}\right)=\sum_{k=1}^{\infty} B_{k}\left(\frac{\alpha_{s}}{\pi}\right)^{k},
$$

where $A_{1}=C_{F}$ and $B_{1}=-3 C_{F} / 2$. The coefficient functions $C^{\text {in }}$ can be written as

$$
C_{a i}^{\mathrm{in}}\left(x ; \mu=b_{0} / b\right)=\delta_{a i} \delta(1-x)+\sum_{k=1}^{\infty} C_{a i}^{\mathrm{in}(k)}(x)\left(\frac{\alpha_{s}}{\pi}\right)^{k},
$$

and an analogous expansion holds for $C^{\text {out }}$. Using the DGLAP equation we can evolve $f_{1}$ from the scale $\mu=b_{0} / b$ to $\mu=Q$ and obtain

$$
f_{1}^{a}\left(x ; b_{0} / b\right)=f_{1}^{a}(x ; Q)-\frac{\alpha_{s}}{2 \pi}\left(P_{q q} \otimes f_{1}^{a}+P_{q g} \otimes f_{1}^{g}\right)(x) \ln \frac{b^{2} Q^{2}}{b_{0}^{2}}+O\left(\alpha_{s}^{2}\right) .
$$

Evolving $D_{1}$ in the same way and putting everything together, we obtain

$$
\begin{aligned}
F_{U U, T}= & \frac{1}{z^{2}} \sum_{a} x e_{a}^{2} \int \frac{d^{2} \boldsymbol{b}}{(2 \pi)^{2}} e^{-i \boldsymbol{b} \cdot \boldsymbol{q}_{T}}\left[1-\frac{\alpha_{s}}{2 \pi} C_{F}\left(\ln ^{2} \frac{b^{2} Q^{2}}{b_{0}^{2}}-3 \ln \frac{b^{2} Q^{2}}{b_{0}^{2}}\right)\right] \\
& \times\left[f_{1}^{a}(x ; Q)-\frac{\alpha_{s}}{2 \pi}\left(P_{q q} \otimes f_{1}^{a}+P_{q g} \otimes f_{1}^{g}\right)(x) \ln \frac{b^{2} Q^{2}}{b_{0}^{2}}+\frac{\alpha_{s}}{\pi} \sum_{i}\left(C_{a i}^{\text {in }(1)} \otimes f_{1}^{i}\right)(x)\right] \\
& \times\left[D_{1}^{a}(z ; Q)-\frac{\alpha_{s}}{2 \pi}\left(D_{1}^{a} \otimes P_{q q}+D_{1}^{g} \otimes P_{g q}\right)(z) \ln \frac{b^{2} Q^{2}}{b_{0}^{2}}+\frac{\alpha_{s}}{\pi} \sum_{j}\left(D_{1}^{j} \otimes C_{j a}^{\text {out }}(1)\right)(z)\right] \\
& +O\left(\alpha_{s}^{2}\right) .
\end{aligned}
$$

The running of $\alpha_{s}$ is irrelevant at the accuracy of this expression. Expanding the square brackets one obtains a term $f_{1}^{a}(x ; Q) D_{1}^{a}(z ; Q)$ of order $\alpha_{s}^{0}$, which is independent of $b$ and hence gives a contribution proportional to $\delta^{(2)}\left(\boldsymbol{q}_{T}\right)$ to $F_{U U, T}$. Since we require $q_{T} \gg M$, this term must be discarded. For the same reason, the first-order coefficients $C_{a i}^{\text {in }}(1)$ and $C_{j a}^{\text {out (1) }}$ do not contribute to $F_{U U, T}$ at order $\alpha_{s}$. With the integrals 30]

$$
\int d^{2} \boldsymbol{b} e^{-i \boldsymbol{b} \cdot \boldsymbol{q}_{T}} \ln ^{2} \frac{b^{2} Q^{2}}{b_{0}^{2}}=-\frac{8 \pi}{q_{T}^{2}} \ln \frac{Q^{2}}{q_{T}^{2}}, \quad \int d^{2} \boldsymbol{b} e^{-i \boldsymbol{b} \cdot \boldsymbol{q}_{T}} \ln \frac{b^{2} Q^{2}}{b_{0}^{2}}=-\frac{4 \pi}{q_{T}^{2}}
$$

we recover the lowest-order result (3.12) from (3.19). Going to higher orders in $\alpha_{s}$, one finds that the term with $A_{1}$ in the Sudakov factor produces the leading logarithms $\alpha_{s}^{k} \ln ^{2 k-1}\left(Q^{2} / q_{T}^{2}\right)$ in $F_{U U, T}$, whereas the next-to-leading logarithms $\alpha_{s}^{k} \ln ^{2 k-2}\left(Q^{2} / q_{T}^{2}\right)$ also receive contributions from the coefficient $B_{1}$, from the one-loop running of $\alpha_{s}$, and from the leading-order evolution of $f_{1}(x)$ and $D_{1}(z)$. 
The $\alpha_{s}$ expansion of the CSS factorization formula (3.14), which we have just performed to leading order, allows one to determine the functions $S, C^{\text {in }}$ and $C^{\text {out }}$ at a given order in perturbation theory by comparing with the collinear fixed-order calculation in the high- $q_{T}$ region. The functional form of (3.14) was however derived by CSS using the result of CS factorization in the intermediate region $M \ll q_{T} \ll Q$. This is not immediately obvious by comparing (3.14) with (3.10), because in the former expression there is no hard and no soft factor. As pointed out in [31], one can however introduce the hard factor into the CSS expression. $|H(\mu=Q)|^{2}$ depends on $Q$ only through the argument of $\alpha_{s}$, so that one can use the renormalization group equation for the running coupling to rewrite it as $|H(\mu=Q)|^{2}=\left|H\left(\mu=b_{0} / b\right)\right|^{2} e^{R(Q, b)}$. Since $C^{\text {in }}$ and $C^{\text {out }}$ in (3.14) are also evaluated at $\mu=b_{0} / b$, we can combine factors into $C^{\prime \text { in }}=|H|^{-1} C^{\text {in }}$ and $C^{\prime \text { out }}=|H|^{-1} C^{\text {out }}$ at that scale, which gives

$$
\begin{aligned}
F_{U U, T}= & |H(\mu=Q)|^{2} \frac{1}{z^{2}} \sum_{a} x e_{a}^{2} \int \frac{d^{2} \boldsymbol{b}}{(2 \pi)^{2}} e^{-i \boldsymbol{b} \cdot \boldsymbol{q}_{T}} \exp \left[-S^{\prime}(Q, b)\right] \\
& \times \sum_{i}\left(C_{a i}^{\prime \text { in }} \otimes f_{1}^{i}\right)\left(x ; \mu=b_{0} / b\right) \sum_{j}\left(D_{1}^{j} \otimes C_{j a}^{\text {out }}\right)\left(z ; \mu=b_{0} / b\right) .
\end{aligned}
$$

with $S^{\prime}=S+R$. Identifying $C^{\prime \text { in }}=\widetilde{U}^{1 / 2} \widehat{C}^{\text {in }}, C^{\text {out }}=\widetilde{U}^{1 / 2} \widehat{C}^{\text {out }}$, and $S^{\prime}=\widehat{S}$, we finally recognize the CS result (3.10).

The upshot of this discussion is essential in our context: the CSS derivation of transverse momentum resummation for $F_{U U, T}$ and its analogs in $e^{+} e^{-}$annihilation and Drell-Yan production makes use of two facts:

1. CS factorization is valid for these observables at $q_{T} \ll Q$, and

2. in the intermediate region $M \ll q_{T} \ll Q$ its results match those obtained from collinear factorization in the high- $q_{T}$ region.

In such a situation one can go further and construct expressions that interpolate between the factorization formulae for low and for high $q_{T}$ and are thus valid for all $q_{T}$, from $q_{T}=0$ to $q_{T} \sim Q$. A prescription for this had already been given by CSS, and a number of different methods have been proposed later; see [32] for a discussion and references. We shall not dwell on this issue here.

\subsection{Azimuthal dependence and polarization}

So far we have discussed only $F_{U U, T}$. The situation for the other unpolarized structure functions, $F_{U U, L}, F_{U U}^{\cos \phi_{h}}, F_{U U}^{\cos 2 \phi_{h}}$, cannot readily be inferred from the results of CSS. In section 1 we will see that the splitting functions appearing in the analogs of (3.12) for these three structure functions differ from the usual ones. Since the splitting functions are relevant at next-to-leading logarithmic accuracy, it is not clear if and how resummation beyond the leading logarithmic approximation can be performed in this case. An analogous observation for the angular distribution in Drell-Yan production has been made in [9]. (Resummation at leading logarithmic accuracy has recently been considered in [10].) 
The extension of CSS factorization for polarized scattering is relatively straightforward as long as one integrates over the azimuthal angle of $\boldsymbol{q}_{T}$. For SIDIS this concerns the structure function $F_{L L}$, and a corresponding calculation in this framework has been presented in [32]. CSS resummation for Drell-Yan production with longitudinal beam polarization has been investigated in [33, 34]. A detailed discussion of polarization in the context of collinear factorization can be found in [35].

In the present work we will apply CS factorization to polarized scattering and to the SIDIS cross section depending on the azimuth $\phi_{h}$, as has been done in [36]. The factors $\not P f_{1}^{a}\left(x, p_{T}^{2} ; \zeta\right)$ and $\not P_{h} D_{1}^{a}\left(z, k_{T}^{2} ; \zeta_{h}\right)$ in the factorization formula (3.2) are then replaced by the quark-quark correlator $\Phi^{a}\left(x, \boldsymbol{p}_{T} ; \zeta\right)$ and the fragmentation correlator $\Delta^{a}\left(z, \boldsymbol{k}_{T} ; \zeta_{h}\right)$, which will be defined in section 5.1. The result reads

$$
\begin{aligned}
W_{\text {SIDIS }}^{\mu \nu} \propto & \left|H\left(x \zeta^{1 / 2}, z^{-1} \zeta_{h}^{1 / 2}\right)\right|^{2} \sum_{a} e_{a}^{2} \int d^{2} \boldsymbol{p}_{T} d^{2} \boldsymbol{k}_{T} d^{2} \boldsymbol{l}_{T} \delta^{(2)}\left(\boldsymbol{p}_{T}-\boldsymbol{k}_{T}+\boldsymbol{l}_{T}+\boldsymbol{q}_{T}\right) \\
& \times \operatorname{Tr}\left\{\Phi^{a}\left(x, \boldsymbol{p}_{T} ; \zeta\right) \gamma^{\mu} \Delta^{a}\left(z, \boldsymbol{k}_{T} ; \zeta_{h}\right) \gamma^{\nu}\right\} U\left(l_{T}^{2}\right)
\end{aligned}
$$

and gives rise to a number of spin and azimuthal asymmetries at leading order in $1 / Q$. It is an open question if and how CS factorization can be extended to power suppressed observables, at least to those coming with one factor of $1 / Q$. The calculations of our work are relevant to this question, as we shall see in section 8.3 .

A structure function that has received much attention in the literature is $F_{U T, T}^{\sin \left(\phi_{h}-\phi_{S}\right)}$, which arises when the initial hadron is transversely polarized. At low $q_{T}$ this observable is nonzero due to the Sivers effect [37]: the CS factorization formula (3.22) gives a leading contribution in $1 / Q$ proportional to the Sivers function $f_{1 T}^{\perp}\left(x, p_{T}^{2}\right)$ [12]. At high $q_{T}$ one can describe the same observable in terms of the Qiu-Sterman mechanism [38]. The corresponding calculation uses collinear factorization at twist-three level, i.e., $F_{U T, T}^{\sin \left(\phi_{h}-\phi_{S}\right)}$ is suppressed by $1 / q_{T}$ compared with $F_{U U, T}$. Calculating the behavior of the Sivers function at high transverse momentum, the analysis in [7, 8] has shown that at order $\alpha_{s}$ the two descriptions exactly match in the intermediate region $M \ll q_{T} \ll Q$. The situation is the same for the corresponding asymmetry in Drell-Yan production [5, 6, 8]. This suggests that for $F_{U T, T}^{\sin \left(\phi_{h}-\phi_{S}\right)}$ and its Drell-Yan analog it should be possible to use the CSS resummation procedure for large logarithms of $Q / q_{T}$, as discussed in [39].

In the following sections we will derive the power behavior for the full set of SIDIS structure functions, both in the low- $q_{T}$ and in the high- $q_{T}$ description. This will in particular determine whether or not one can envisage to use CSS resummation for these observables. To determine the power behavior we can restrict our calculations to the leading order in $\alpha_{s}$. Since we will not attempt to actually perform a resummation of large logarithms, we need not go to $b$-space as in (3.10) or (3.14). We will instead directly work with the momentumspace version (3.22) of CS factorization. In particular, we shall recover the fixed-order result (3.12) for the intermediate region when expanding the CS expression (3.3) of $F_{U U, T}$ in the limit $q_{T} \gg M$. 


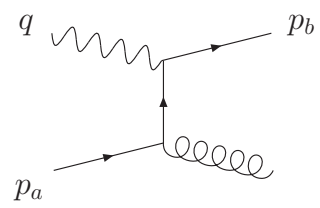

(a)

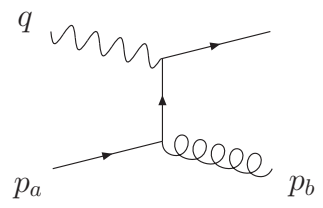

(b)

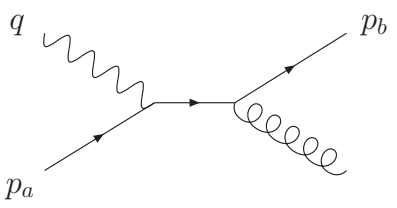

$\left(a^{\prime}\right)$

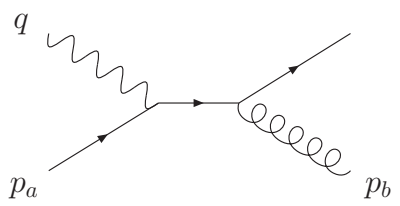

$\left(b^{\prime}\right)$

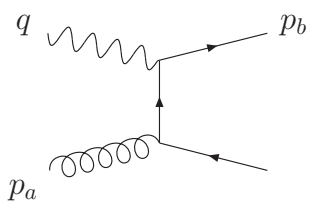

(c)

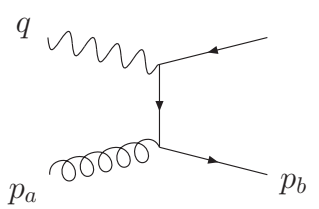

$\left(c^{\prime}\right)$

Figure 2: Feynman diagrams for the processes $\gamma^{*} q \rightarrow q g, \gamma^{*} q \rightarrow g q$, and $\gamma^{*} g \rightarrow q \bar{q}$.

\section{From high to intermediate $q_{T}$}

In this section we present the calculation of SIDIS structure functions at high transverse momentum in terms of collinear distribution and fragmentation functions, and then take the limit $q_{T} \ll Q$. We give explicit results for the six structure functions appearing at leading twist and order $\alpha_{s}$. For seven of the remaining structure functions, which are of higher twist or higher order in $\alpha_{s}$, there exist studies in the literature, which we will briefly discuss.

In the high- $q_{T}$ calculation, the generation of large transverse momentum is described by hard-scattering processes at parton level. The diagrams for the contributions at first order in $\alpha_{s}$ are shown in figure 2. We introduce the scaling variables

$$
\hat{x}=\frac{Q^{2}}{2 p_{a} \cdot q}, \quad \hat{z}=\frac{p_{a} \cdot p_{b}}{p_{a} \cdot q}
$$

for the partonic subprocess, where $p_{a}$ is the momentum of the incoming parton and $p_{b}$ the momentum of the parton which fragments into the observed hadron $h$. We furthermore use the transverse momentum $q_{T}^{\mu}$ introduced in (2.6). Neglecting mass corrections we have $\hat{x} p_{a}=x P$ and $p_{b} / \hat{z}=P_{h} / z$, so that

$$
q_{T}^{\mu}=q^{\mu}+(1-r) \hat{x} p_{a}^{\mu}-p_{b}^{\mu} / \hat{z}
$$

with $r=q_{T}^{2} / Q^{2}$. The partonic Mandelstam variables are then given by

$$
\begin{aligned}
& \hat{s}=\left(q+p_{a}\right)^{2}=\frac{1-\hat{x}}{\hat{x}} Q^{2}, \quad \hat{t}=\left(q-p_{b}\right)^{2}=-\frac{1-\hat{z}}{\hat{x}} Q^{2}=-\frac{\hat{z}}{1-\hat{x}} q_{T}^{2}, \\
& \hat{u}=\left(p_{a}-p_{b}\right)^{2}=-\frac{\hat{z}}{\hat{x}} Q^{2} .
\end{aligned}
$$

The structure functions defined in section 2 can be written as convolutions of hard- 
scattering coefficients with collinear parton distribution and fragmentation functions,

$$
\begin{aligned}
F_{U U, T} & =\frac{1}{Q^{2}} \frac{\alpha_{s}}{(2 \pi z)^{2}} \sum_{a} x e_{a}^{2} \int_{x}^{1} \frac{d \hat{x}}{\hat{x}} \int_{z}^{1} \frac{d \hat{z}}{\hat{z}} \delta\left(\frac{q_{T}^{2}}{Q^{2}}-\frac{(1-\hat{x})(1-\hat{z})}{\hat{x} \hat{z}}\right) \\
\times & {\left[f_{1}^{a}\left(\frac{x}{\hat{x}}\right) D_{1}^{a}\left(\frac{z}{\hat{z}}\right) C_{U U, T}^{\left(\gamma^{*} q \rightarrow q g\right)}+f_{1}^{a}\left(\frac{x}{\hat{x}}\right) D_{1}^{g}\left(\frac{z}{\hat{z}}\right) C_{U U, T}^{\left(\gamma^{*} q \rightarrow g q\right)}+f_{1}^{g}\left(\frac{x}{\hat{x}}\right) D_{1}^{a}\left(\frac{z}{\hat{z}}\right) C_{U U, T}^{\left(\gamma^{*} g \rightarrow q \bar{q}\right)}\right], }
\end{aligned}
$$

as we have already seen in section 3.2. We recall that $a$ runs over flavors of quarks and of antiquarks. Analogous expressions with different kernels $C$ give the structure functions $F_{U U, L}, F_{U U}^{\cos \phi_{h}}$, and $F_{U U}^{\cos 2 \phi_{h}}$. At order $\alpha_{s}$ (but not at higher order) one finds the relation

$$
F_{U U, L}=2 F_{U U}^{\cos 2 \phi_{h}} .
$$

The structure functions $F_{L L}$ and $F_{L L}^{\cos \phi_{h}}$ for longitudinal target and beam polarization are also given by expressions analogous to (4.4), with different kernels $C$ and with the unpolarized parton densities $f_{1}^{a}$ and $f_{1}^{g}$ replaced by their polarized counterparts $g_{1}^{a}$ and $g_{1}^{g}$. The hard-scattering coefficients for the partonic processes $\gamma^{*} q \rightarrow q g, \gamma^{*} q \rightarrow g q, \gamma^{*} g \rightarrow q \bar{q}$ can be computed from the respective diagrams $\left(a, a^{\prime}\right),\left(b, b^{\prime}\right),\left(c, c^{\prime}\right)$ in figure 2 , and those for $\gamma^{*} \bar{q} \rightarrow \bar{q} g, \gamma^{*} \bar{q} \rightarrow g \bar{q}, \gamma^{*} g \rightarrow \bar{q} q$ are identical to their counterparts obtained by charge conjugation. Process by process we have

- $\gamma^{*} q \rightarrow q g$

$$
\begin{aligned}
C_{U U, T} & =2 C_{F}\left((1-\hat{x})(1-\hat{z})+\frac{1+\hat{x}^{2} \hat{z}^{2}}{\hat{x} \hat{z}} \frac{Q^{2}}{q_{T}^{2}}\right), \\
C_{U U}^{\cos \phi_{h}} & =-4 C_{F}[\hat{x} \hat{z}+(1-\hat{x})(1-\hat{z})] \frac{Q}{q_{T}} \\
C_{U U}^{\cos 2 \phi_{h}} & =4 C_{F} \hat{x} \hat{z}, \\
C_{L L} & =2 C_{F}\left(2(\hat{x}+\hat{z})+\frac{\hat{x}^{2}+\hat{z}^{2}}{\hat{x} \hat{z}} \frac{Q^{2}}{q_{T}^{2}}\right), \\
C_{L L}^{\cos \phi_{h}} & =-4 C_{F}(\hat{x}+\hat{z}-1) \frac{Q}{q_{T}},
\end{aligned}
$$

- $\gamma^{*} q \rightarrow g q$

$$
\begin{aligned}
C_{U U, T} & =2 C_{F}\left((1-\hat{x}) \hat{z}+\frac{1+\hat{x}^{2}(1-\hat{z})^{2}}{\hat{x} \hat{z}} \frac{1-\hat{z}}{\hat{z}} \frac{Q^{2}}{q_{T}^{2}}\right), \\
C_{U U}^{\cos \phi_{h}} & =4 C_{F}[\hat{x}(1-\hat{z})+(1-\hat{x}) \hat{z}] \frac{1-\hat{z}}{\hat{z}} \frac{Q}{q_{T}}, \\
C_{U U}^{\cos 2 \phi_{h}} & =4 C_{F} \hat{x}(1-\hat{z}), \\
C_{L L} & =2 C_{F}\left(2 \hat{x}+2(1-\hat{z})+\frac{\hat{x}^{2}+(1-\hat{z})^{2}}{\hat{x} \hat{z}} \frac{1-\hat{z}}{\hat{z}} \frac{Q^{2}}{q_{T}^{2}}\right), \\
C_{L L}^{\cos \phi_{h}} & =4 C_{F}(\hat{x}-\hat{z}) \frac{1-\hat{z}}{\hat{z}} \frac{Q}{q_{T}},
\end{aligned}
$$


- $\gamma^{*} g \rightarrow q \bar{q}$

$$
\begin{aligned}
C_{U U, T} & =2 T_{R}\left[\hat{x}^{2}+(1-\hat{x})^{2}\right]\left[\hat{z}^{2}+(1-\hat{z})^{2}\right] \frac{1-\hat{x}}{\hat{x} \hat{z}^{2}} \frac{Q^{2}}{q_{T}^{2}} \\
C_{U U}^{\cos \phi_{h}} & =-4 T_{R}(2 \hat{x}-1)(2 \hat{z}-1) \frac{1-\hat{x}}{\hat{z}} \frac{Q}{q_{T}} \\
C_{U U}^{\cos 2 \phi_{h}} & =8 T_{R} \hat{x}(1-\hat{x}) \\
C_{L L} & =2 T_{R}(2 \hat{x}-1)\left[\hat{z}^{2}+(1-\hat{z})^{2}\right] \frac{1-\hat{x}}{\hat{x} \hat{z}^{2}} \frac{Q^{2}}{q_{T}^{2}}, \\
C_{L L}^{\cos \phi_{h}} & =-4 T_{R}(2 \hat{z}-1) \frac{1-\hat{x}}{\hat{z}} \frac{Q}{q_{T}}
\end{aligned}
$$

with $C_{F}=4 / 3$ and $T_{R}=1 / 2$. The relation $C_{U U, L}=2 C_{U U}^{\cos 2 \phi_{h}}$ holds for each individual subprocess. Our results agree with those in [40, 32].

The behavior of the above results in the region $q_{T}^{2} \ll Q^{2}$ can be obtained by rewriting the $\delta$ function in eq. (4.4) as 41]

$$
\begin{aligned}
\delta\left(\frac{q_{T}^{2}}{Q^{2}}-\frac{(1-\hat{x})(1-\hat{z})}{\hat{x} \hat{z}}\right)= & \delta(1-\hat{x}) \delta(1-\hat{z}) \ln \frac{Q^{2}}{q_{T}^{2}}+\frac{\hat{x}}{(1-\hat{x})_{+}} \delta(1-\hat{z}) \\
& +\frac{\hat{z}}{(1-\hat{z})_{+}} \delta(1-\hat{x})+\mathcal{O}\left(\frac{q_{T}^{2}}{Q^{2}} \ln \frac{Q^{2}}{q_{T}^{2}}\right)
\end{aligned}
$$

where the plus-distribution is as usual defined by

$$
\int_{z}^{1} d y \frac{G(y)}{(1-y)_{+}}=\int_{z}^{1} d y \frac{G(y)-G(1)}{1-y}-G(1) \ln \frac{1}{1-z} .
$$

We have written the hard-scattering coefficients in (4.6) to (4.20) in a way that allows for an easy extraction of the leading power behavior at small $q_{T} / Q$. The result is

$$
\begin{aligned}
& F_{U U, T}=\frac{1}{q_{T}^{2}} \frac{\alpha_{s}}{2 \pi^{2} z^{2}} \sum_{a} x e_{a}^{2}\left[f_{1}^{a}(x) D_{1}^{a}(z) L\left(\frac{Q^{2}}{q_{T}^{2}}\right)+f_{1}^{a}(x)\left(D_{1}^{a} \otimes P_{q q}+D_{1}^{g} \otimes P_{g q}\right)(z)\right. \\
& \left.+\left(P_{q q} \otimes f_{1}^{a}+P_{q g} \otimes f_{1}^{g}\right)(x) D_{1}^{a}(z)\right], \\
& F_{U U, L}=2 F_{U U}^{\cos 2 \phi_{h}} \\
& F_{U U}^{\cos \phi_{h}}=-\frac{1}{Q q_{T}} \frac{\alpha_{s}}{2 \pi^{2} z^{2}} \sum_{a} x e_{a}^{2}\left[f_{1}^{a}(x) D_{1}^{a}(z) L\left(\frac{Q^{2}}{q_{T}^{2}}\right)+f_{1}^{a}(x)\left(D_{1}^{a} \otimes P_{q q}^{\prime}+D_{1}^{g} \otimes P_{g q}^{\prime}\right)(z)\right. \\
& \left.+\left(P_{q q}^{\prime} \otimes f_{1}^{a}+P_{q g}^{\prime} \otimes f_{1}^{g}\right)(x) D_{1}^{a}(z)\right], \\
& F_{U U}^{\cos 2 \phi_{h}}=\frac{1}{Q^{2}} \frac{\alpha_{s}}{2 \pi^{2} z^{2}} \sum_{a} x e_{a}^{2}\left[f_{1}^{a}(x) D_{1}^{a}(z) L\left(\frac{Q^{2}}{q_{T}^{2}}\right)+f_{1}^{a}(x)\left(D_{1}^{a} \otimes P_{q q}^{\prime \prime}+D_{1}^{g} \otimes P_{g q}^{\prime \prime}\right)(z)\right. \\
& \left.+\left(P_{q q}^{\prime \prime} \otimes f_{1}^{a}+P_{q g}^{\prime \prime} \otimes f_{1}^{g}\right)(x) D_{1}^{a}(z)\right],
\end{aligned}
$$


and

$$
\begin{gathered}
F_{L L}=\frac{1}{q_{T}^{2}} \frac{\alpha_{s}}{2 \pi^{2} z^{2}} \sum_{a} x e_{a}^{2}\left[g_{1}^{a}(x) D_{1}^{a}(z) L\left(\frac{Q^{2}}{q_{T}^{2}}\right)+g_{1}^{a}(x)\left(D_{1}^{a} \otimes P_{q q}+D_{1}^{g} \otimes P_{g q}\right)(z)\right. \\
\left.+\left(\Delta P_{q q} \otimes g_{1}^{a}+\Delta P_{q g} \otimes g_{1}^{g}\right)(x) D_{1}^{a}(z)\right] \\
F_{L L}^{\cos \phi_{h}}=-\frac{1}{Q q_{T}} \frac{\alpha_{s}}{2 \pi^{2} z^{2}} \sum_{a} x e_{a}^{2}\left[g_{1}^{a}(x) D_{1}^{a}(z) L\left(\frac{Q^{2}}{q_{T}^{2}}\right)+g_{1}^{a}(x)\left(D_{1}^{a} \otimes P_{q q}^{\prime}+D_{1}^{g} \otimes P_{g q}^{\prime}\right)(z)\right. \\
\left.+\left(\Delta P_{q q}^{\prime} \otimes g_{1}^{a}+\Delta P_{q g}^{\prime} \otimes g_{1}^{g}\right)(x) D_{1}^{a}(z)\right]
\end{gathered}
$$

The factor $L$ contains a logarithm of $Q^{2} / q_{T}^{2}$ and is given in (3.13), and the convolutions are defined in (3.9). The splitting functions are given by

$$
\begin{array}{ll}
P_{q q}(\hat{x})=C_{F}\left[\frac{1+\hat{x}^{2}}{(1-\hat{x})_{+}}+\frac{3}{2} \delta(1-\hat{x})\right], \quad P_{q g}(\hat{x})=T_{R}\left[\hat{x}^{2}+(1-\hat{x})^{2}\right], \\
P_{g q}(\hat{z})=C_{F} \frac{1+(1-\hat{z})^{2}}{\hat{z}}, & \\
P_{q q}^{\prime}(\hat{x})=C_{F}\left[\frac{2 \hat{x}^{2}}{(1-\hat{x})_{+}}+\frac{3}{2} \delta(1-\hat{x})\right], & P_{q g}^{\prime}(\hat{x})=2 T_{R} \hat{x}(2 \hat{x}-1), \\
P_{g q}^{\prime}(\hat{z})=-2 C_{F}(1-\hat{z}), & \\
P_{q q}^{\prime \prime}(\hat{x})=P_{q q}^{\prime}(\hat{x}), & P_{q g}^{\prime \prime}(\hat{x})=4 T_{R} \hat{x}^{2}, \\
P_{g q}^{\prime \prime}(\hat{z})=2 C_{F} \hat{z} &
\end{array}
$$

for convolutions with unpolarized distribution or fragmentation functions, and by

$$
\begin{aligned}
& \Delta P_{q q}(\hat{x})=P_{q q}(\hat{x}), \quad \Delta P_{q g}(\hat{x})=T_{R}(2 \hat{x}-1), \\
& \Delta P_{q q}^{\prime}(\hat{x})=P_{q q}^{\prime}(\hat{x}), \quad \Delta P_{q g}^{\prime}(\hat{x})=2 T_{R} \hat{x}
\end{aligned}
$$

for convolutions with polarized distributions. Similar results for the Drell-Yan process have been obtained by Boer and Vogelsang in ref. [9]. We note that our $P_{q q}^{\prime}$ and $P_{q q}^{\prime \prime}$ correspond to $P_{q q}^{-}$in eq. (38) of ref. [9], while our $P_{q g}^{\prime}$ corresponds to their $\tilde{P}_{q g}^{-}$and our $P_{q g}^{\prime \prime}$ to their $P_{q g}^{\prime-} / 2$.

Let us remark that the $1 / q_{T}$ power behavior of $F_{U U}^{\cos \phi_{h}}$ and $F_{L L}^{\cos \phi_{h}}$ arises as $q_{T} / q_{T}^{2}$, where $1 / q_{T}^{2}$ comes from the $t$-channel propagators in the hard-scattering graphs of figure 2 . Likewise, the constant $q_{T}$ behavior (up to $\ln q_{T}^{2}$ terms) of $F_{U U, L}$ and $F_{U U}^{\cos 2 \phi_{h}}$ arises as $q_{T}^{2} / q_{T}^{2}$, where the $1 / q_{T}^{2}$ from the hard propagators is fully canceled by numerator factors. We emphasize that, although they do not have a power-law divergence, the above expressions for $F_{U U, L}$ and $F_{U U}^{\cos 2 \phi_{h}}$ cannot be used for small $q_{T}$, because the approximations giving $1 / q_{T}^{2}$ for the hard propagators break down when $q_{T} \lesssim M$. In fact, angular momentum conservation requires $F_{U U}^{\cos \phi_{h}}$ and $F_{L L}^{\cos \phi_{h}}$ to vanish like $q_{T}$ and $F_{L L}^{\cos 2 \phi_{h}}$ to vanish like $q_{T}^{2}$ for $q_{T} \rightarrow 0$, as shown e.g. in [42]. 
The six $F_{U T}$ structure functions for transverse target polarization vanish in the leadingtwist approximation at high transverse momentum, because they would require the combination of the transversity distribution function $h_{1}$ with a chiral-odd collinear fragmentation function of twist two, which does not exist for an unpolarized hadron. They are however nonzero at twist three, where collinear quark-gluon-quark and three-gluon correlation functions appear, so that many more diagrams than the ones in figure 2 need to be computed. Such a computation has been performed by Eguchi, Koike and Tanaka in refs. [43, 44]. The results for the different structure functions involve the product of $D_{1}$ with $G_{F}$ and $\widetilde{G}_{F}$, which are chiral-even functions appearing in the decomposition of the quark-gluon-quark distribution correlator given in (5.37). Some observables involve in addition the product of $h_{1}$ with $\widehat{E}_{F}$, which is a chiral-odd function appearing in the decomposition of the quarkgluon-quark fragmentation correlator. The structure function $F_{U T, T}^{\sin \left(\phi_{h}-\phi_{S}\right)}$ has also been computed in [7]. The result differs from the one in [43, 44] because both calculations were missing certain terms. With the corrections discussed in [8], agreement between the two groups has been achieved. We note, however, that the twist-three calculation of the $F_{U T}$ structure functions is presently not complete. Terms involving $\widehat{E}_{F}$ are only considered in [43], where they are found to contribute to $F_{U T, T}^{\sin \left(\phi_{h}-\phi_{S}\right)}, F_{U T}^{\sin \left(\phi_{h}+\phi_{S}\right)}$, and $F_{U T}^{\sin \phi_{S}}$. The calculation of that work is restricted to so-called derivative terms due to soft gluon pole contributions. The remaining soft gluon pole contributions, as well as contributions from soft fermion poles and hard poles are evaluated in [44], but only for $G_{F}$ and $\widetilde{G}_{F}$. In [8] it is shown that soft fermion pole contributions from further diagrams must be added to those results. Finally, all calculations in the literature are restricted to quark-gluon-quark functions of twist three, so that three-gluon correlators do not appear.

Using the soft gluon pole and the hard pole contributions computed in 443, 44] we have extracted the leading behavior of all $F_{U T}$ structure functions in the limit $q_{T} \ll Q$. The structure of the results is listed in eqs. (6.8) to (6.13) of section 6, both for the power law and for the distribution and fragmentation functions appearing in each observable. We have verified that this structure is not changed by the soft fermion pole contributions given in [44]. Since the corrections to [44] discussed in [8] concern only soft fermion pole contributions in $F_{U T}^{\sin \left(\phi_{h}+\phi_{S}\right)}$ they do not affect the structure of (6.10) either. The same should hold for the remaining five $F_{U T}$ structure functions, but this has not been checked explicitly.

The structure function $F_{L U}^{\sin \phi_{h}}$ is nonzero at twist two and order $\alpha_{s}^{2}$. Depending on a single polarization, it is a $T$-odd observable and hence requires an absorptive part in the amplitude, which in this case is provided by a loop in the hard-scattering subprocess. The relevant graphs have been calculated in [45], and numerical estimates for specific kinematics have been given in [45, 46]. For the structure functions $F_{U L}^{\sin \phi_{h}}$ and $F_{U L}^{\sin 2 \phi_{h}}$ the situation is similar, but no explicit calculation exists in the literature. There is no contribution to $F_{L U}^{\sin \phi_{h}}, F_{U L}^{\sin \phi_{h}}, F_{U L}^{\sin 2 \phi_{h}}$ at twist three and order $\alpha_{s}$, because the necessary $T$-odd terms would need to come from twist-three quark-gluon-quark correlators. For an unpolarized or longitudinally polarized hadron, these are chiral-odd [43] and have no twist-two chiral-odd partners in the other correlator. From the calculation in [45] we can extract the power behavior of $F_{L U}^{\sin \phi_{h}}$ for $q_{T} \ll Q$, which we will give in eq. (6.5). 


\section{From low to intermediate $q_{T}$ : power counting}

In this section we derive the behavior of distribution and fragmentation functions at high transverse momentum. Plugging the results into the known low- $q_{T}$ expressions of the SIDIS structure functions, we will obtain their power behavior in the intermediate region $M \ll q_{T} \ll Q$. To begin with, we specify in the next two subsections the distribution and fragmentation functions that will appear in our calculation.

\subsection{Transverse-momentum-dependent distribution and fragmentation func- tions}

For the discussion of distribution and fragmentation functions we use light-cone coordinates defined with respect to the momenta $P$ and $P_{h}$, which we already introduced at the end of section 2. For any four-vector $a$ we then have the plus-component $a^{+}=a \cdot n_{-}$, the minus-component $a^{-}=a \cdot n_{+}$, and the transverse part $a_{T}^{\mu}=a^{\mu}-a^{+} n_{+}^{\mu}-a^{-} n_{-}^{\mu}$. The hadron momenta read

$$
P^{\mu}=P^{+} n_{+}^{\mu}+\frac{M^{2}}{2 P^{+}} n_{-}^{\mu}, \quad P_{h}^{\mu}=P_{h}^{-} n_{-}^{\mu}+\frac{M_{h}^{2}}{2 P_{h}^{-}} n_{+}^{\mu},
$$

and the spin vector of the target can be decomposed into longitudinal and transverse components as

$$
S^{\mu}=S_{L}\left(\frac{P^{+}}{M} n_{+}^{\mu}-\frac{M}{2 P^{+}} n_{-}^{\mu}\right)+S_{T}^{\mu} .
$$

The transverse-momentum-dependent quark distributions appearing in the description of SIDIS are defined from the quark-quark correlation function

$$
\Phi_{i j}^{[\mathcal{U}]}\left(x, p_{T}\right)=\left.\int \frac{d \xi^{-} d^{2} \boldsymbol{\xi}_{T}}{(2 \pi)^{3}} e^{i p \cdot \xi}\left\langle P\left|\bar{\psi}_{j}(0) \mathcal{U}_{(0, \xi)} \psi_{i}(\xi)\right| P\right\rangle\right|_{\xi^{+}=0},
$$

where $p^{+}=x P^{+}$and summation over color indices is understood. The corresponding correlation function for antiquarks is obtained by replacing the quark fields by their transforms under charge conjugation, see ref. [11]. The quark fields in (5.3) are renormalized fields, and the corresponding scale dependence of the correlation function is given by a renormalization group equation involving the quark anomalous dimension [24].

The gauge link $\mathcal{U}_{(0, \xi)}$ in (5.3) is a Wilson line that connects the quark fields and thus makes the correlation function color gauge invariant. In the factorization theorems for scattering processes, the gauge link incorporates the exchange of gluons between partons that move in the opposite light-cone directions $n_{+}$and $n_{-}$. Consideration of gluons collinear to the target yields Wilson lines with paths that point along $n_{-}$and lead to light-cone infinity, $a^{-}= \pm \infty$, where they are closed by transverse segments from $\mathbf{0}_{T}$ to $\boldsymbol{\xi}_{T}$ [47, 13]. Different processes require different gauge links. In particular, the simplest links closed at $a^{-}= \pm \infty$, which we denote by $\mathcal{U}^{ \pm}$, give rise to the correlators $\Phi^{[+]}\left(x, p_{T}\right)$ and $\Phi^{[-]}\left(x, p_{T}\right)$ appearing in SIDIS and Drell-Yan production, respectively. More complicated gauge links show up in other processes [48, 49]. 
When defined with strictly lightlike Wilson lines, the correlator (5.3) contains divergences in gluon rapidity (sometimes referred to as "endpoint singularities") and hence must be modified [25]. Different schemes have been discussed in the literature. One possibility is to use paths that point in a non-lightlike direction $v$ instead of $n_{-}$[26, 27]. Up to subtle issues we will mention in appendix $\mathrm{A}$, this is equivalent to working in axial gauge, $A \cdot v=0$, which was used in the original scheme of Collins and Soper 24]. In a number of different schemes, the proton matrix element in (5.3) is divided by vacuum expectation values of suitably chosen Wilson lines [27, 50-52]. The arguments in the present section use Lorentz invariance and power counting, so that we need not specify the detailed choice of scheme. As long as $v$ is a linear superposition of $n_{+}$and $n_{-}$, no new four-vector is introduced in $\Phi\left(x, p_{T}\right)$, which therefore depends on $v$ only via the scalar parameter $\zeta=-(2 P \cdot v)^{2} / v^{2}$ we already encountered in section 3.1.

The correlation function (5.3) can be parameterized in terms of distributions functions depending on $x$ and $p_{T}^{2}$ as 14

$$
\begin{aligned}
& \Phi\left(x, p_{T}\right) \\
& =\frac{1}{2}\left\{f_{1} \not \not_{+}+f_{1 T}^{\perp} \frac{S_{T \rho} \epsilon_{T}^{\rho \sigma} p_{T \sigma}}{M} \not \not_{+}+g_{1 L} S_{L} \gamma_{5} \not \not_{+}-g_{1 T} \frac{S_{T} \cdot p_{T}}{M} \gamma_{5} \not \not_{+}\right. \\
& \left.+h_{1} \frac{\gamma_{5}\left[\not \not_{T}, \not \not_{+}\right]}{2}-h_{1 T}^{\perp} \frac{S_{T \rho} p_{T}^{(\rho} p_{T}^{\sigma)}}{M^{2}} \frac{\gamma_{5}\left[\gamma_{\sigma}, \not \not_{+}\right]}{2}+h_{1 L}^{\perp} S_{L} \frac{\gamma_{5}\left[\not p_{T}, \not h_{+}\right]}{2 M}+h_{1}^{\perp} \frac{i\left[\not p_{T}, \not \not_{+}\right]}{2 M}\right\} \\
& +\frac{M}{2 P^{+}}\left\{e-e_{L} S_{L} i \gamma_{5}+e_{T} \frac{S_{T} \cdot p_{T}}{M} i \gamma_{5}+e_{T}^{\perp} \frac{S_{T \rho} \epsilon_{T}^{\rho \sigma} p_{T \sigma}}{M}\right. \\
& +f^{\perp} \frac{\not p_{T}}{M}+f_{L}^{\perp} S_{L} \frac{p_{T \rho} \epsilon_{T}^{\rho \sigma}}{M} \gamma_{\sigma}-f_{T}^{\perp} \frac{S_{T \rho} p_{T}^{(\rho} p_{T}^{\sigma)} \epsilon_{T \sigma \tau}}{M^{2}} \gamma^{\tau}+f_{T} S_{T \rho} \epsilon_{T}^{\rho \sigma} \gamma_{\sigma} \\
& +g_{L}^{\perp} S_{L} \frac{\gamma_{5} \not p_{T}}{M}+g^{\perp} \frac{p_{T \rho} \epsilon_{T}^{\rho \sigma}}{M} \gamma_{5} \gamma_{\sigma}-g_{T}^{\perp} \frac{S_{T \rho} p_{T}^{(\rho} p_{T}^{\sigma)}}{M^{2}} \gamma_{5} \gamma_{\sigma}+g_{T} \gamma_{5} \$_{T}^{\star} \\
& \left.+h_{T}^{\perp} \frac{\gamma_{5}\left[\$_{T}, \not \phi_{T}\right]}{2 M}-h_{T} \frac{S_{T} \cdot p_{T}}{M} \frac{\gamma_{5}\left[\not h_{+}, \not h_{-}\right]}{2}+h_{L} S_{L} \frac{\gamma_{5}\left[\not h_{+}, \not h_{-}\right]}{2}+h \frac{i\left[\not h_{+}, \not h_{-}\right]}{2}\right\} \\
& +\frac{M^{2}}{2\left(P^{+}\right)^{2}}\{\ldots\} \text {, }
\end{aligned}
$$

where the two-dimensional antisymmetric tensor is given by

$$
\epsilon_{T}^{\alpha \beta}=\epsilon^{\alpha \beta \rho \sigma} n_{+\rho} n_{-\sigma}
$$

with $\epsilon^{0123}=1$. Index pairs in parentheses indicate that the trace is subtracted in the two transverse dimensions,

$$
p_{T}^{(\rho} p_{T}^{\sigma)}=p_{T}^{\rho} p_{T}^{\sigma}-\frac{1}{2}\left(p_{T} \cdot p_{T}\right) g_{T}^{\rho \sigma},
$$

where the transverse metric tensor is $g_{T}^{\alpha \beta}=g^{\alpha \beta}-n_{+}^{\alpha} n_{-}^{\beta}-n_{-}^{\alpha} n_{+}^{\beta}$. The first eight distributions in (5.4) are referred to as twist two, and the next sixteen distributions as twist three. The ... stand for the remaining eight distributions of twist four, which are given in [53]. 


\begin{tabular}{|c|ccc|ccc|cccccc|}
\hline & \multicolumn{10}{|c|}{ target polarization } \\
\cline { 2 - 12 } & \multicolumn{10}{|c|}{ unpolarized } & \multicolumn{10}{|c|}{ longitudinal } & \multicolumn{1}{c|}{ transverse } \\
\hline & $f_{1}$ & $f^{\perp}$ & $g^{\perp}$ & $g_{1 L}$ & $g_{L}^{\perp}$ & $f_{L}^{\perp}$ & $f_{1 T}^{\perp}$ & $f_{T}^{\perp}$ & $f_{T}$ & $g_{1 T}$ & $g_{T}^{\perp}$ & $g_{T}$ \\
$\eta_{f}$ & + & + & - & + & + & - & - & - & - & + & + & + \\
$n$ & 2 & 2 & 2 & 2 & 2 & 2 & 4 & 4 & 2 & 4 & 4 & 2 \\
\hline & $h_{1}^{\perp}$ & $h$ & $e$ & $h_{1 L}^{\perp}$ & $h_{L}$ & $e_{L}$ & $h_{1}$ & $h_{1 T}^{\perp}$ & $h_{T}$ & $h_{T}^{\perp}$ & $e_{T}$ & $e_{T}^{\perp}$ \\
$\eta_{f}$ & - & - & + & + & + & - & + & + & + & + & - & - \\
$n$ & 4 & 2 & 2 & 4 & 2 & 2 & 2 & 4 & 2 & 2 & 2 & 2 \\
\hline
\end{tabular}

Table 1: Behavior of distribution functions under time reversal and in the high- $p_{T}$ limit. The time reversal factor $\eta_{f}$ is defined in (5.7) and the exponent $n$ for the high- $p_{T}$ behavior in (5.8).

We will not need them in the following and tacitly omit them in further parameterizations. Corresponding to the Dirac matrix structure in the decomposition (5.4), functions denoted with letters $f, g$ or $e, h$ are respectively referred to as chiral-even or chiral-odd. Functions with subscripts $L$ or $T$ appear in the parts of $\Phi\left(x, p_{T}\right)$ that depend on the longitudinal or transverse component of the spin vector. (An exception to this rule of notation is the transversity distribution $h_{1}$.)

It is understood that the correlator and each of the functions in (5.4) should carry a label specifying the gauge link, as well as a label for the quark flavor. Time reversal connects $\Phi^{[\mathcal{U}]}$ with $\Phi^{\left[\mathcal{U}^{T}\right]}$, and in particular $\Phi^{[+]}$with $\Phi^{[-]}$. This provides relations [54]

$$
f^{[+]}\left(x, p_{T}^{2}\right)=\eta_{f} f^{[-]}\left(x, p_{T}^{2}\right),
$$

where $f$ stands for any of the distributions in (5.4). We call a distribution $T$-even if $\eta_{f}=+1$ and $T$-odd if $\eta_{f}=-1$. The values of $\eta_{f}$ are given in table 1. We also anticipate in the table the power behavior

$$
f^{[ \pm]}\left(x, p_{T}^{2}\right) \sim 1 / p_{T}^{n}
$$

of the distributions for $p_{T} \gg M$, which we shall derive in section 5.3 .

Fragmentation functions are defined from the correlator

$$
\begin{aligned}
\Delta_{i j}\left(z, k_{T}\right)=\frac{1}{2 N_{c} z} \sum_{X} & \int \frac{d \xi^{+} d^{2} \boldsymbol{\xi}_{T}}{(2 \pi)^{3}} e^{i k \cdot \xi} \\
& \times\left.\left\langle 0\left|\mathcal{U}_{(\infty, \xi)} \psi_{i}(\xi)\right| h, X\right\rangle_{\text {out out }}\left\langle h, X\left|\bar{\psi}_{j}(0) \mathcal{U}_{(0, \infty)}\right| 0\right\rangle\right|_{\xi^{-}=0},
\end{aligned}
$$

where $k^{-}=P_{h}^{-} / z$ and $N_{c}=3$. The prefactor $1 /\left(2 N_{c}\right)$ comes from averaging over the polarization and color of the fragmenting quark. The subscript $\infty$ in the gauge links indicates a space-time point with plus-coordinate $a^{+}=\infty$. The precise choice of Wilson lines involves the same issues we mentioned for the distribution correlator. Aspects specific to the case of fragmentation functions are discussed in [50, 49]. Notice that fragmentation functions with different gauge links are not related by time reversal, because time reversal 
transforms "out" states $|h, X\rangle_{\text {out }}$ into "in" states $|h, X\rangle_{\text {in }}$, and the difference between these states amounts in general to more than just a phase.

For an unpolarized hadron $h$ the decomposition of the fragmentation correlator reads

$$
\begin{aligned}
\Delta\left(z, k_{T}\right)= & \frac{1}{2}\left\{D_{1} \not \not_{-}+H_{1}^{\perp} \frac{i\left[\not \not_{T}, \not \not_{-}\right]}{2 M_{h}}\right\} \\
& +\frac{M_{h}}{2 P_{h}^{-}}\left\{E+D^{\perp} \frac{\not \not_{T}}{M_{h}}+H \frac{i\left[\not \not_{-}, \not \not_{+}\right]}{2}-G^{\perp} \frac{k_{T \rho} \epsilon_{T}^{\rho \sigma}}{M_{h}} \gamma_{5} \gamma_{\sigma}\right\},
\end{aligned}
$$

where the functions on the r.h.s. depend on $z$ and $k_{T}^{2}$. In a more explicit notation they should also carry a flavor index.

\subsection{Collinear distribution and fragmentation functions}

In applications of collinear factorization, the structure of incoming hadrons is represented by the light-cone distribution correlator

$$
\Phi_{i j}(x)=\left.\int \frac{d \xi^{-}}{2 \pi} e^{i p \cdot \xi}\left\langle P\left|\bar{\psi}_{j}(0) \mathcal{U}_{(0, \xi)}^{n_{-}} \psi_{i}(\xi)\right| P\right\rangle\right|_{\xi^{+}=0, \boldsymbol{\xi}_{T}=\mathbf{0}_{T}},
$$

where the gauge link $\mathcal{U}_{(0, \xi)}^{n-}$ connects the quark fields along a path in the $n_{-}$direction. This would simply be the integral of the $p_{T}$-dependent correlator introduced in the previous subsection,

$$
\Phi(x)=\int d^{2} \boldsymbol{p}_{T} \Phi^{[\mathcal{U}]}\left(x, p_{T}\right),
$$

were it not for two complications. On the one hand, the correlator (5.11) has ultraviolet divergences due to the fact that all field operators are taken at the same transverse position. Their regularization and subtraction gives rise to a scale dependence described by the DGLAP equations. Correspondingly, the integrand on the r.h.s. of (5.12) diverges like $1 / p_{T}^{2}$ at high $p_{T}$, as we will see in the next subsection, so that the $p_{T}$-integral must be regularized. On the other hand, the rapidity divergences of the $p_{T}$-dependent correlator, which we discussed in the previous subsection, cancel under the integral over $p_{T}$ [25, 51]. They require no regularization in the collinear correlation function (5.11), which hence is independent of the parameter $\zeta$. The different regularization procedures in the correlators $\Phi\left(x, p_{T}\right)$ and $\Phi(x)$ reflect the different types of subtractions required when constructing transverse-momentum-dependent or collinear factorization theorems. We will shortly discuss how the relation (5.12) should be understood.

The correlation function (5.11) can be parameterized as

$$
\begin{aligned}
\Phi(x)=\frac{1}{2}\left\{f_{1} \not h_{+}\right. & \left.+g_{1} S_{L} \gamma_{5} \not h_{+}+h_{1} \frac{\gamma_{5}\left[\phi_{T}, \not h_{+}\right]}{2}\right\}+\frac{M}{2 P^{+}}\left\{e-e_{L} S_{L} i \gamma_{5}\right. \\
& \left.+f_{T} S_{T \rho} \epsilon_{T}^{\rho \sigma} \gamma_{\sigma}+g_{T} \gamma_{5} \phi_{T}+h_{L} S_{L} \frac{\gamma_{5}\left[\not \not_{+}, \not h_{-}\right]}{2}+h \frac{i\left[\not \not_{+}, \not_{-}\right]}{2}\right\},
\end{aligned}
$$

where the distributions on the r.h.s. depend only on $x$. They are given by

$$
f_{1}(x)=\int d^{2} \boldsymbol{p}_{T} f_{1}\left(x, p_{T}^{2}\right)
$$


and similarly for the other functions, with one common exception of notation,

$$
g_{1}(x)=\int d^{2} \boldsymbol{p}_{T} g_{1 L}\left(x, p_{T}^{2}\right) .
$$

Since the gauge link in (5.11) is along a finite light-like path from 0 to $\xi$, time reversal relates the collinear correlator with itself, and as a consequence $f_{T}(x)=e_{L}(x)=h(x)=0$. This ensures that (5.12) can simultaneously hold for different links $\mathcal{U}$, in particular for $\mathcal{U}^{+}$ and $\mathcal{U}^{-}$, which according to (5.7) give $p_{T^{-}}$dependent distributions $f_{T}^{[ \pm]}, e_{L}^{[ \pm]}$, and $h^{[ \pm]}$of opposite sign.

To make the meaning of (5.12) more precise, we observe that the combination of eqs. (3.5) and (3.8) gives

$$
\tilde{f}_{1}(x, b ; \zeta, \mu)=\int d^{2} \boldsymbol{p}_{T} e^{i \boldsymbol{b} \cdot \boldsymbol{p}_{T}} f_{1}\left(x, p_{T}^{2} ; \zeta, \mu\right)=f_{1}(x ; \mu)+O\left(\alpha_{s} \ln ^{2}\left(\zeta b^{2}\right)\right)
$$

for small enough $b$, where we have set $\mu=b_{0} / b$ and used the perturbative expansions $\widehat{S}^{\prime}=O\left(\alpha_{s} \ln ^{2}\left(\zeta b^{2}\right)\right)$ and $\widehat{C}_{a i}^{\text {in }}=\delta_{a i} \delta(1-x)+O\left(\alpha_{s}\right)$. The factor $e^{i \boldsymbol{b} \cdot \boldsymbol{p}_{T}}$ in (5.16) regulates the logarithmic divergence of $\int d^{2} \boldsymbol{p}_{T} f_{1}\left(x, p_{T}^{2}\right)$ by damping the integrand for large $p_{T}>1 / b$. Alternatively one can cut off the integral at $p_{T}=b_{0} / b$, since

$$
\int d^{2} \boldsymbol{p}_{T} e^{i \boldsymbol{b} \cdot \boldsymbol{p}_{T}} f_{1}\left(x, p_{T}^{2} ; \zeta, \mu\right)=\int d^{2} \boldsymbol{p}_{T} \theta\left(\mu^{2}-\boldsymbol{p}_{T}^{2}\right) f_{1}\left(x, p_{T}^{2} ; \zeta, \mu\right)
$$

up to corrections of order $b^{2}$, as we will show in appendix B. We thus see that the relation (5.12) should be understood with a suitable regulator of the integral on the r.h.s. and as up to corrections of order $\alpha_{s}$. The same holds for (5.14), (5.15), and for similar integral relations in the following. Let us remark that an extension of (5.16) to the full correlation function $\Phi$ has not been given in the literature.

As we will see in the next subsection, calculations at subleading power or those involving azimuthal asymmetries lead in the collinear expansion to $p_{T}$-weighted correlation functions

$$
\Phi_{\partial}^{\alpha[ \pm]}(x)=\int d^{2} \boldsymbol{p}_{T} p_{T}^{\alpha} \Phi^{[ \pm]}\left(x, p_{T}\right),
$$

where the Lorentz index $\alpha$ is restricted to be transverse, and where the same remarks about regularization apply as for $(5.12)$. In contrast to $\Phi(x)$, the correlator $\Phi_{\partial}^{\alpha[\mathcal{U}]}(x)$ does depend on the choice of Wilson lines in $\Phi^{[\mathcal{U}]}\left(x, p_{T}\right)$ and hence contains both $T$-even and $T$-odd distributions. Omitting the superscript $[\mathcal{U}]$ for the sake of legibility, we have the decomposition

$$
\begin{aligned}
\Phi_{\partial}^{\alpha}(x)=-\frac{M}{2}\{ & f_{1 T}^{\perp(1)} S_{T \rho} \epsilon_{T}^{\rho \alpha} \not \not_{+}-g_{1 T}^{(1)} S_{T}^{\alpha} \gamma_{5} \not h_{+} \\
& \left.+h_{1 L}^{\perp(1)} S_{L} \frac{\gamma_{5}\left[\gamma_{T}^{\alpha}, \not \not_{+}\right]}{2}+h_{1}^{\perp(1)} \frac{i\left[\gamma_{T}^{\alpha}, \not \not_{+}\right]}{2}\right\}+\frac{M^{2}}{2 P^{+}}\{\ldots\},
\end{aligned}
$$

where we have only displayed the terms of leading twist and defined $\boldsymbol{p}_{T}^{2}$ moments

$$
f_{1 T}^{\perp(n)}(x)=\int d^{2} \boldsymbol{p}_{T}\left(\frac{\boldsymbol{p}_{T}^{2}}{2 M^{2}}\right)^{n} f_{1 T}^{\perp}\left(x, p_{T}^{2}\right),
$$


and similarly for the other functions. The functions $f_{1 T}^{\perp(n)}$ and $h_{1}^{\perp(n)}$ are $T$-odd and thus change sign when going from $\Phi_{\partial}^{\alpha[+]}(x)$ to $\Phi_{\partial}^{\alpha[-]}(x)$.

The factor $p_{T}^{\alpha}$ in (5.18) can be converted into a derivative $\partial^{\alpha}$ acting on the matrix element that appears in the definition (5.3) of $\Phi\left(x, p_{T}\right)$. One can then express $\Phi_{\partial}^{\alpha}(x)$ in terms of correlators with either a gluon field or a covariant derivative between the antiquark and quark fields. The former is a collinear quark-antiquark-gluon correlation function, whereas the latter can be rewritten in terms of the quark-quark correlator $\Phi(x)$ using the equation of motion for the quark field. In this way, the $\boldsymbol{p}_{T}^{2}$ moments given in (5.19) can be traded for functions of twist three, up to twist-two distributions multiplied by the quark mass [14.

The $k_{T}$-integrated fragmentation correlator for an unpolarized hadron has the decomposition

$$
\Delta(z)=z^{2} \int d^{2} \boldsymbol{k}_{T} \Delta\left(z, k_{T}\right)=\frac{1}{2} D_{1} \not h_{-}+\frac{M_{h}}{2 P_{h}^{-}}\left\{E+H \frac{i\left[\not h_{-}, \not h_{+}\right]}{2}\right\},
$$

where the fragmentation functions on the r.h.s. depend on $z$. They are given by

$$
D_{1}(z)=z^{2} \int d^{2} \boldsymbol{k}_{T} D_{1}\left(z, k_{T}^{2}\right)
$$

and similarly for the other functions. Notice the factor $z^{2}$, which appears because $D_{1}\left(z, k_{T}^{2}\right)$ is a probability density w.r.t. the transverse momentum $k_{T}^{\prime}=-z k_{T}$ of the final-state hadron relative to the fragmenting quark [55, 11]. As already discussed, time reversal invariance does not constrain fragmentation correlators, so that $H(z)$ can be nonzero unlike its distribution counterpart $h(x)$. For the $k_{T}$-weighted correlation function needed in calculations at twist three and higher, we have

$$
\Delta_{\partial}^{\alpha}(z)=z^{2} \int d^{2} \boldsymbol{k}_{T} k_{T}^{\alpha} \Delta\left(z, k_{T}\right)=-\frac{M_{h}}{2} H_{1}^{\perp(1)} \frac{i\left[\gamma_{T}^{\alpha}, \not h_{-}\right]}{2}+\frac{M_{h}^{2}}{2 P_{h}^{-}}\{\ldots\}
$$

with

$$
H_{1}^{\perp(n)}(z)=z^{2} \int d^{2} \boldsymbol{k}_{T}\left(\frac{\boldsymbol{k}_{T}^{2}}{2 M_{h}^{2}}\right)^{n} H_{1}^{\perp}\left(z, k_{T}^{2}\right),
$$

where again $\alpha$ is restricted to be transverse.

\subsection{Distribution and fragmentation functions at high transverse momentum}

We are now ready to derive the behavior of correlation functions at high transverse momentum. We consider the distribution correlator $\Phi\left(x, p_{T}\right)$ for transverse momentum $p_{T}$ much larger than the scale of nonperturbative interactions. The generation of the large transverse momentum can be described in perturbation theory. Technically, we approximate $\Phi\left(x, p_{T}\right)$ in powers of $1 / p_{T}$ using a collinear expansion that leads to the factorization of the transverse momentum dependence. To derive a formal proof of factorization, one would use the same techniques as for, say, the production of a high- $p_{T}$ jet in deep inelastic scattering. We shall not attempt this here, but limit ourselves to determining the power-law behavior of the distribution functions that parameterize $\Phi\left(x, p_{T}\right)$, using Lorentz invariance and dimensional analysis as our main tools. The explicit calculation in section 8 


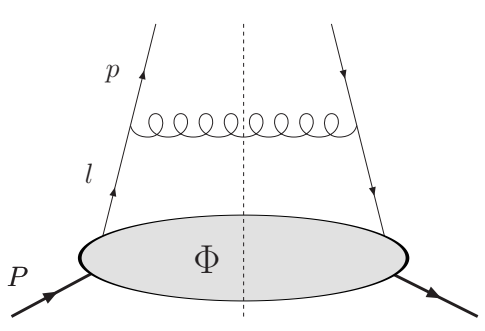

(a)

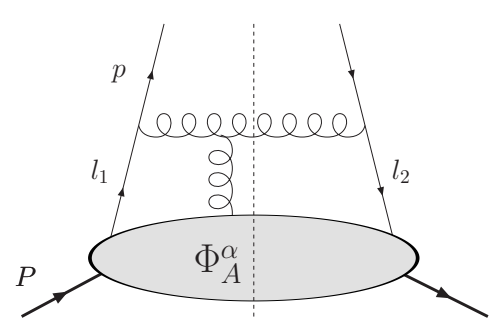

(b)

Figure 3: Example diagrams for the calculation of the high- $p_{T}$ behavior of the quark-quark correlator $\Phi\left(x, p_{T}\right)$. The dashed lines represent the final-state cut.

and the one for the Sivers function $f_{1 T}^{\perp}$ in [6, 8] provide examples for the consistency of the collinear factorization formalism at leading order in $\alpha_{s}$ and to leading and first subleading power in $1 / p_{T}$. One should, however, be aware that factorization might break down at some higher-order or higher-power accuracy.

The evaluation of $\Phi\left(x, p_{T}\right)$ at lowest order in $1 / p_{T}$ involves diagrams as in figure ba, whereas at higher orders correlators with three or more partons appear at the bottom of the graphs, as shown in figure $3 \mathrm{~b} \mathrm{~b}$. To set up the power counting, we generically write $p$ for the hard scale and take

$$
\begin{aligned}
p^{+}, p^{-}, \boldsymbol{p}_{T} \sim P^{+} \sim l^{+}, l_{1}^{+}, l_{2}^{+} & \sim p \\
\boldsymbol{l}_{T}, \boldsymbol{l}_{1 T}, \boldsymbol{l}_{2 T} & \sim M, \\
P^{-} \sim l^{-}, l_{1}^{-}, l_{2}^{-} & \sim M^{2} / p,
\end{aligned}
$$

where the mass $M$ represents the soft scale. In (5.27) we have used that the loop momenta $l, l_{1}$ and $l_{2}$ are attached to a soft function at the bottom of the graphs and thus have virtualities of order $M^{2}$. Starting point of the calculation is the correlation function depending on the full four-momentum $p$,

$$
\Phi_{i j}^{[ \pm]}(p)=\int \frac{d^{4} \xi}{(2 \pi)^{4}} e^{i p \cdot \xi}\left\langle P\left|\bar{\psi}_{j}(0) \mathcal{U}_{(0, \xi)}^{ \pm} \psi_{i}(\xi)\right| P\right\rangle
$$

from which we obtain $\Phi^{[ \pm]}\left(x, p_{T}\right)=\int d p^{-} \Phi^{[ \pm]}(p)$. We omit the superscript $[ \pm]$ for clarity of notation in the next few steps, and will restore it when required later on. We restrict ourselves to the leading and first subleading order of $\Phi\left(x, p_{T}\right)$ in the $1 / p$ expansion. To this order, the relevant factorized graphs can be written as the convolution of hard-scattering kernels and correlation functions in the form

$$
\begin{aligned}
\Phi\left(x, p_{T}\right)= & \int d p^{-} d^{4} l H(p, l) \Phi(l)+\int d p^{-} d^{4} l_{1} d^{4} l_{2} H_{A}^{\alpha}\left(p, l_{1}, l_{2}\right) \Phi_{A \alpha}\left(l_{1}, l_{2}\right) \\
& +\{\text { terms with two- and three-gluon correlators }\}+\cdots
\end{aligned}
$$

with $\ldots$ representing terms of higher order in $1 / p$. It is understood that the hard-scattering kernels $H(p, l)$ and $H_{A}^{\alpha}\left(p, l_{1}, l_{2}\right)$ include $\delta$ functions putting the cut lines on shell — this 
can readily be used to perform the integration over $p^{-}$. The lower blob in figure $3 \mathrm{~b}$ is parameterized by the quark-gluon-quark correlator $\Phi_{A}^{\alpha}$, which contains the gluon potential $A^{\alpha}$ between the quark and antiquark fields. The gluon polarization index $\alpha$ in (5.29) is restricted to be transverse: the contribution from $A^{-}$gluons is power suppressed by at least $1 / p^{2}$, whereas the corresponding contribution of $A^{+}$gluons ends up in the gauge link of the quark-quark correlator when the terms in the factorization formula are arranged in a gauge-invariant manner.

We now expand the hard-scattering kernels in the small momentum components (5.26) and (5.27). To the order we are considering, we can neglect $l_{1}^{-}, l_{2}^{-}$and $l_{1 T}, l_{2 T}$ in $H_{A}^{\alpha}\left(p, l_{1}, l_{2}\right)$, whereas in $H(p, l)$ we can neglect $l^{-}$but must expand the $l_{T^{-}}$-dependence up to first order. This gives

$$
\begin{aligned}
\int d p^{-} H(p, l) & =\frac{1}{p^{+}} \hat{x} H_{2}\left(\hat{x}, p^{+}, p_{T}\right)+\frac{l_{T \alpha}}{p^{+}} \hat{x} H_{3}^{\alpha}\left(\hat{x}, p^{+}, p_{T}\right)+\cdots, \\
\int d p^{-} H_{A}^{\alpha}\left(p, l_{1}, l_{2}\right) & =\frac{1}{p^{+}} \hat{x}_{1} \hat{x}_{2} H_{A, 3}^{\alpha}\left(\hat{x}_{1}, \hat{x}_{2}, p^{+}, p_{T}\right)+\cdots,
\end{aligned}
$$

where we have introduced the plus-momentum fractions

$$
\hat{x}=p^{+} / l^{+}, \quad \hat{x}_{1}=p^{+} / l_{1}^{+}, \quad \hat{x}_{2}=p^{+} / l_{2}^{+}
$$

and chosen prefactors such that $H_{2}, H_{3}^{\alpha}$ and $H_{A, 3}^{\alpha}$ are invariant under longitudinal boosts, i.e. under the rescaling (2.5) of $n_{ \pm}$and the corresponding change of plus- and minus components. The convolution (5.29) now takes the form

$$
\begin{aligned}
\Phi(x, & \left.p_{T}\right) \\
= & \int \frac{d \hat{x}}{\hat{x}} H_{2}\left(\hat{x}, p^{+}, p_{T}\right) \int d l^{-} d^{2} \boldsymbol{l}_{T} \Phi(l)+\int \frac{d \hat{x}}{\hat{x}} H_{3}^{\alpha}\left(\hat{x}, p^{+}, p_{T}\right) \int d l^{-} d^{2} \boldsymbol{l}_{T} l_{T \alpha} \Phi(l) \\
& +p^{+} \int \frac{d \hat{x}_{1}}{\hat{x}_{1}} \frac{d \hat{x}_{2}}{\hat{x}_{2}} H_{A, 3}^{\alpha}\left(\hat{x}_{1}, \hat{x}_{2}, p^{+}, p_{T}\right) \int d l_{1}^{-} d l_{2}^{-} d^{2} \boldsymbol{l}_{1 T} d^{2} \boldsymbol{l}_{2 T} \Phi_{A \alpha}\left(l_{1}, l_{2}\right) \\
& +\{\text { terms with two- and three-gluon correlators }\}+\cdots .
\end{aligned}
$$

In the first two terms we recognize the collinear quark-quark correlators from 5.12 and (5.18),

$$
\Phi(y)=\int d l^{-} d^{2} \boldsymbol{l}_{T} \Phi(l), \quad \Phi_{\partial}^{\alpha}(y)=\int d l^{-} d^{2} \boldsymbol{l}_{T} l_{T}^{\alpha} \Phi(l)
$$

with $y=l^{+} / P^{+}$, whereas the third term involves a collinear quark-gluon-quark correlation function

$$
\Phi_{A}^{\alpha}\left(y_{1}, y_{2}\right)=\int d l_{1}^{-} d l_{2}^{-} d^{2} \boldsymbol{l}_{1 T} d^{2} \boldsymbol{l}_{2 T} \Phi_{A}^{\alpha}\left(l_{1}, l_{2}\right)
$$

with $y_{1}=l_{1}^{+} / P^{+}$and $y_{2}=l_{2}^{+} / P^{+}$. In order for these correlators to be gauge invariant one must reshuffle certain pieces among the different terms in (5.33), as shown for instance in [13]. On the r.h.s. of (5.34) and (5.35) this implies subtraction of terms with the gluon 
potential $A^{\alpha}$ at light-cone infinity, which we have not displayed. One also finds that taking the gauge link $\mathcal{U}^{ \pm}$in $\Phi^{[ \pm]}\left(x, p_{T}\right)$ leads to the corresponding path-dependent correlators $\Phi_{\partial}^{\alpha[ \pm]}(y)$ and

$$
\Phi_{A}^{\alpha[ \pm]}\left(y_{1}, y_{2}\right)=\frac{1}{i P^{+}} \frac{\Phi_{G}^{\alpha}\left(y_{1}, y_{2}\right)}{y_{1}-y_{2} \pm i \epsilon}
$$

in the factorization formula, where

$$
\begin{aligned}
\Phi_{G i j}^{\alpha}\left(y_{1}, y_{2}\right)= & \int \frac{d \xi_{1}^{-}}{2 \pi} \frac{d \xi_{2}^{-}}{2 \pi} e^{i l_{1} \cdot \xi_{1}} e^{i\left(l_{2}-l_{1}\right) \cdot \xi_{2}} \\
& \times\left.\left\langle P\left|\bar{\psi}_{j}(0) \mathcal{U}_{\left(0, \xi_{2}\right)}^{n_{-}} g G^{+\alpha}\left(\xi_{2}\right) \mathcal{U}_{\left(\xi_{2}, \xi_{1}\right)}^{n_{-}} \psi_{i}\left(\xi_{1}\right)\right| P\right\rangle\right|_{\xi_{1}^{+}=\xi_{2}^{+}=0, \boldsymbol{\xi}_{1 T}=\boldsymbol{\xi}_{2 T}=\mathbf{0}_{T}}
\end{aligned}
$$

does not carry a superscript $[ \pm]$ because, like $\Phi(y)$, it involves only Wilson lines of finite length along $n_{-}$. We now decompose the correlation functions into terms of definite twist,

$$
\begin{aligned}
\Phi(y) & =\Phi_{2}(y)+\frac{M}{P^{+}} \Phi_{3}(y)+\cdots, \quad \Phi_{\partial}^{\alpha}(y)=M \Phi_{\partial, 3}^{\alpha}(y)+\cdots, \\
\Phi_{A}^{\alpha}\left(y_{1}, y_{2}\right) & =\frac{M}{P^{+}} \Phi_{A, 3}^{\alpha}\left(y_{1}, y_{2}\right)+\cdots,
\end{aligned}
$$

where the prefactors are chosen such that $\Phi_{2}, \Phi_{3}$ and $\Phi_{\partial, 3}, \Phi_{A, 3}$ are dimensionless and independent of $P^{+}$. Under a longitudinal boost $\Phi_{3}$ is invariant, whereas the other correlators transform like $n_{+}$. Dimensional counting readily gives $H_{2} \sim 1 / p^{2}$ and $H_{3}, H_{A, 3} \sim 1 / p^{3}$. Using $p^{+}=x P^{+}$and (5.32) we can then rewrite (5.33) as

$$
\begin{aligned}
\Phi\left(x, p_{T}\right)= & \int \frac{d \hat{x}}{\hat{x}} H_{2}\left(\hat{x}, p^{+}, p_{T}\right) \Phi_{2}\left(\frac{x}{\hat{x}}\right) \\
& +M\left\{\int \frac{d \hat{x}}{\hat{x}} \frac{H_{2}\left(\hat{x}, p^{+}, p_{T}\right)}{p^{+}} x \Phi_{3}\left(\frac{x}{\hat{x}}\right)+\int \frac{d \hat{x}}{\hat{x}} H_{3}^{\alpha}\left(\hat{x}, p^{+}, p_{T}\right) \Phi_{\partial, 3 \alpha}\left(\frac{x}{\hat{x}}\right)\right. \\
& \left.\quad+\int \frac{d \hat{x}_{1}}{\hat{x}_{1}} \frac{d \hat{x}_{2}}{\hat{x}_{2}} H_{A, 3}^{\alpha}\left(\hat{x}_{1}, \hat{x}_{2}, p^{+}, p_{T}\right) x \Phi_{A, 3 \alpha}\left(\frac{x}{\hat{x}_{1}}, \frac{x}{\hat{x}_{2}}\right)\right\} \\
& +\{\text { terms with two- and three-gluon correlators }\}+\mathcal{O}\left(1 / p^{4}\right),
\end{aligned}
$$

where the first term is of order $1 / p^{2}$ and the terms with prefactor $M$ are of order $1 / p^{3}$. To obtain the high- $p_{T}$ behavior of the individual distribution functions parameterizing $\Phi\left(x, p_{T}\right)$, we need to analyze the dependence of the hard-scattering kernels on $p^{+}$and $p_{T}$. The kernels carry four Dirac indices, so that (5.39) explicitly reads

$$
\Phi_{i j}\left(x, p_{T}\right)=\int \frac{d \hat{x}}{\hat{x}} H_{2, i j k l}\left(\hat{x}, p^{+}, p_{T}\right) \Phi_{2, k l}\left(\frac{x}{\hat{x}}\right)+\cdots,
$$

and similarly for the terms of order $1 / p^{3}$. We can decompose $H_{2}$ as

$$
\begin{aligned}
H_{2}\left(\hat{x}, p^{+}, p_{T}\right)=\frac{1}{\boldsymbol{p}_{T}^{2}} & {\left[\sum_{m n} \Gamma_{m} \otimes \Gamma_{n} t_{m n}\left(\hat{x}, p^{+}, p_{T}\right)+\sum_{m n} \Gamma_{m, \mu} \otimes \Gamma_{n, \nu} t_{m n}^{\mu \nu}\left(\hat{x}, p^{+}, p_{T}\right)\right.} \\
& \left.+\sigma_{\lambda \mu} \sigma_{\nu \rho} t^{\lambda \mu \nu \rho}\left(\hat{x}, p^{+}, p_{T}\right)\right]
\end{aligned}
$$


with $\Gamma_{m} \in\left\{1, \gamma_{5}\right\}$ and $\Gamma_{m, \mu} \in\left\{\gamma_{\mu}, \gamma_{\mu} \gamma_{5}\right\}$, where the first matrix in the tensor products carries Dirac indices $i k$ and the second one indices $j l$. Only an even number of $\gamma$ matrices appears in this decomposition, i.e., no structures like $\Gamma_{m, \mu} \otimes \Gamma_{n}$ or $\Gamma_{m, \lambda} \otimes \Gamma_{n, \mu \nu}$, which reflects that chirality is conserved in the hard scattering kernel. The scalars $t_{m n}$ and the tensors $t_{m n}^{\mu \nu}, t^{\lambda \mu \nu \rho}$ are dimensionless and invariant under longitudinal boosts, and therefore they can be constructed from $g_{\mu \nu}, \epsilon_{\lambda \mu \nu \rho}$, and the vectors

$$
\frac{p_{T}^{\mu}}{\left|\boldsymbol{p}_{T}\right|}, \quad \frac{p^{+} n_{+}^{\mu}}{\left|\boldsymbol{p}_{T}\right|}, \quad \frac{\left|\boldsymbol{p}_{T}\right| n_{-}^{\mu}}{p^{+}} .
$$

Since the tensors have an even number of indices, the factors $\left|\boldsymbol{p}_{T}\right|$ combine such that $H_{2}\left(\hat{x}, p^{+}, p_{T}\right)$ depends only on integer powers of $1 / \boldsymbol{p}_{T}^{2}$. The same is readily shown for the kernels $H_{3}^{\alpha}\left(\hat{x}, p^{+}, p_{T}\right)$ and $H_{A, 3}^{\alpha}\left(\hat{x}_{1}, \hat{x}_{2}, p^{+}, p_{T}\right)$, which go like $1 / p^{3}$ instead of $1 / p^{2}$ but involve one more Lorentz index in the analog of the decomposition (5.41). Analogous arguments can be given for the kernels connected with two- or three-gluon correlation functions at the bottom of the graphs (which have two instead of four Dirac indices and two additional Lorentz indices for the exchanged gluons) and for the kernels that appear when $\Phi\left(x, p_{T}\right)$ is evaluated to order $1 / p^{4}$ or higher.

The upshot of this argument is that the distributions parameterizing the correlator $\Phi\left(x, p_{T}\right)$ behave like integer powers of $1 / \boldsymbol{p}_{T}^{2}$ for $\boldsymbol{p}_{T}^{2} \gg M^{2}$. Together with the constraints from dimensional counting and Lorentz invariance, this allows us to determine the leading power behavior of each distribution. Matching the dependence on $p^{+}$, we find for instance that terms on the r.h.s. of (5.39) contribute to the twist-two and twist-three parts of $\Phi\left(x, p_{T}\right)=\Phi_{2}\left(x, p_{T}\right)+\left(M / P^{+}\right) \Phi_{3}\left(x, p_{T}\right)+\cdots$ as

$$
\begin{array}{cl}
\frac{1}{\boldsymbol{p}_{T}^{2}} \rightarrow \Phi_{2}\left(x, p_{T}\right), & \frac{p_{T}^{\rho}}{p^{+} \boldsymbol{p}_{T}^{2}} \rightarrow x \Phi_{3}\left(x, p_{T}\right), \\
\frac{M}{p^{+} \boldsymbol{p}_{T}^{2}} \rightarrow x \Phi_{3}\left(x, p_{T}\right), & \frac{M p_{T}^{\rho}}{\boldsymbol{p}_{T}^{4}} \rightarrow \Phi_{2}\left(x, p_{T}\right),
\end{array}
$$

where we have used that $1 / P^{+}=x / p^{+}$. Comparing with the parameterization (5.4) of $\Phi\left(x, p_{T}\right)$ we see e.g. that $f_{1 T}^{\perp}\left(x, p_{T}^{2}\right)$ and $g_{1 T}\left(x, p_{T}^{2}\right)$ decrease as $M^{2} / \boldsymbol{p}_{T}^{4}$. If there had been terms going like $p_{T}^{\rho} /\left|\boldsymbol{p}_{T}\right|^{3}$ on the r.h.s. of (5.39), they would instead decrease as $M /\left|\boldsymbol{p}_{T}\right|^{3}$.

A number of selection rules specify which collinear distributions can contribute to the high- $p_{T}$ behavior of a given $p_{T}$-dependent distribution. Clearly, the dependence on the target polarization must match. Because the hard scattering conserves chirality, the chirality of distributions must match as well. Finally, we recall that the correlator $\Phi\left(x, p_{T}\right)$ depends on the gauge link and contains terms which are even or odd under the exchange $\Phi^{[+]}\left(x, p_{T}\right) \leftrightarrow \Phi^{[-]}\left(x, p_{T}\right)$. The $T$-odd terms in $\Phi_{\partial}^{[ \pm]}(y)$ and $\Phi_{A}^{[ \pm]}\left(y_{1}, y_{2}\right)$ thus contribute to the $T$-odd distributions in $\Phi^{[ \pm]}\left(x, p_{T}\right)$, and vice versa. The collinear correlator $\Phi(y)$ only contains $T$-even terms, but it can contribute to $T$-odd distributions in $\Phi^{[ \pm]}\left(x, p_{T}\right)$ through graphs with absorptive parts in the hard-scattering subprocess, starting at order $\alpha_{s}^{2}$. An example is shown in figure 6 . Graphs involving the quark-gluon-quark correlator (such as the one in figure $3 \mathrm{~b}$ ) have absorptive parts already at order $\alpha_{s}$, which provides further contributions to the $T$-odd part of $\Phi^{[ \pm]}\left(x, p_{T}\right)$. 


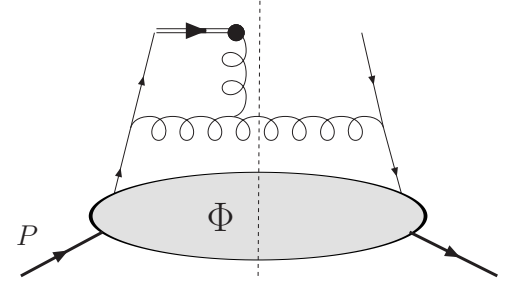

Figure 4: Example of a diagram at order $\alpha_{s}^{2}$, whose absorptive part gives rise to $T$-odd terms in the quark-quark correlator. The double line denotes an eikonal line originating from the gauge link in $\Phi\left(x, p_{T}\right)$, as specified in appendix $\mathrm{A}$.

With the time reversal properties given in table 1, we find that at order $\alpha_{s}$ collinear functions denoted by letters $f, g, e, h$ can only contribute to the high- $p_{T}$ behavior of distributions denoted by the same letter. Putting everything together we find

$$
\begin{array}{rlrlrl}
f_{1} & \sim \frac{1}{\boldsymbol{p}_{T}^{2}} \alpha_{s} \mathcal{F}\left[f_{1}\right], & g_{1 L} & \sim \frac{1}{\boldsymbol{p}_{T}^{2}} \alpha_{s} \mathcal{F}\left[g_{1}\right], & h_{1} & \sim \frac{1}{\boldsymbol{p}_{T}^{2}} \alpha_{s} \mathcal{F}\left[h_{1}\right], \\
x f^{\perp} & \sim \frac{1}{\boldsymbol{p}_{T}^{2}} \alpha_{s} \mathcal{F}\left[f_{1}\right], & x g_{L}^{\perp} & \sim \frac{1}{\boldsymbol{p}_{T}^{2}} \alpha_{s} \mathcal{F}\left[g_{1}\right], & \\
x h_{T} & \sim \frac{1}{\boldsymbol{p}_{T}^{2}} \alpha_{s} \mathcal{F}\left[h_{1}\right], & & x h_{T}^{\perp} & \sim \frac{1}{\boldsymbol{p}_{T}^{2}} \alpha_{s} \mathcal{F}\left[h_{1}\right]
\end{array}
$$

from the $1 / p^{2}$ part of $\Phi\left(x, p_{T}\right)$, and

$$
\begin{aligned}
f_{1 T}^{\perp} & \sim \frac{M^{2}}{\boldsymbol{p}_{T}^{4}} \alpha_{s} \mathcal{F}\left[f_{1 T}^{\perp(1)}, \ldots\right], & g_{1 T} & \sim \frac{M^{2}}{\boldsymbol{p}_{T}^{4}} \alpha_{s} \mathcal{F}\left[g_{1 T}^{(1)}, \ldots\right], \\
h_{1 L}^{\perp} & \sim \frac{M^{2}}{\boldsymbol{p}_{T}^{4}} \alpha_{s} \mathcal{F}\left[h_{1 L}^{\perp(1)}, \ldots\right], & h_{1}^{\perp} & \sim \frac{M^{2}}{\boldsymbol{p}_{T}^{4}} \alpha_{s} \mathcal{F}\left[h_{1}^{\perp(1)}, \ldots\right], \\
x f_{T}^{\perp} & \sim \frac{M^{2}}{\boldsymbol{p}_{T}^{4}} \alpha_{s} \mathcal{F}\left[f_{1 T}^{\perp(1)}, \ldots\right], & x g_{T}^{\perp} & \sim \frac{M^{2}}{\boldsymbol{p}_{T}^{4}} \alpha_{s} \mathcal{F}\left[g_{1 T}^{(1)}, \ldots\right], \\
x f_{T} & \sim \frac{1}{\boldsymbol{p}_{T}^{2}} \alpha_{s} \mathcal{F}\left[f_{1 T}^{\perp(1)}, \ldots\right], & x g_{T} & \sim \frac{1}{\boldsymbol{p}_{T}^{2}} \alpha_{s} \mathcal{F}\left[g_{1 T}^{(1)}, \ldots\right], \\
x h_{L} & \sim \frac{1}{\boldsymbol{p}_{T}^{2}} \alpha_{s} \mathcal{F}\left[h_{1 L}^{\perp(1)}, \ldots\right], & x h & \sim \frac{1}{\boldsymbol{p}_{T}^{2}} \alpha_{s} \mathcal{F}\left[h_{1}^{\perp(1)}, \ldots\right], \\
x e_{L} & \sim \frac{1}{\boldsymbol{p}_{T}^{2}} \alpha_{s} \mathcal{F}[\ldots], & x e & \sim \frac{1}{\boldsymbol{p}_{T}^{2}} \alpha_{s} \mathcal{F}[x e, \ldots]
\end{aligned}
$$

from the $1 / p^{3}$ part of $\Phi\left(x, p_{T}\right)$. The distributions on the l.h.s. depend on $x$ and $p_{T}$, and on the right-hand side we have convolutions of the form

$$
\mathcal{F}[f]=K_{q} \otimes f^{a}+K_{g} \otimes f^{g},
$$

where $a$ is the quark or antiquark flavor of the $p_{T}$-dependent functions on the l.h.s. of (5.44) or (5.45). We note that at higher orders in $\alpha_{s}$ one has instead a sum over all quark and antiquark flavors, as in (3.8). The contribution from gluon distributions in (5.46) is absent for chiral-odd distributions. The kernels $K_{q}$ and $K_{g}$ are of course not the same for the 
different functions in (5.44) and (5.45), and we use $\mathcal{F}$ in a generic sense. We have explicitly calculated the hard-scattering kernels $H_{2}$ and $H_{3}$ defined by (5.30) and verified that they give nonzero contributions for the functions given as arguments of $\mathcal{F}$ in (5.44) and (5.45). By ... we have denoted contributions from three-parton correlation functions. We have not listed the twist-three distributions parameterizing $\Phi_{3}(x)$ as arguments of $\mathcal{F}$, because they can (up to quark mass suppressed terms) be related to the functions in $\Phi_{\partial, 3}(x)$ and to quark-gluon-quark correlation functions, as we remarked after eq. (5.20). An exception is $e(x)$, which has no counterpart in $\Phi_{\partial, 3}(x)$. Furthermore, there is no distribution in $\Phi_{2}$ or $\Phi_{\partial, 3}$ that is multiplied by $S_{L}$ and both $T$-odd and chiral-odd. At order $\alpha_{s}$ and $1 / p^{3}$ the high- $p_{T}$ behavior of $e_{L}\left(x, p_{T}^{2}\right)$ can hence only be generated from the $\Phi_{A, 3}$ term in $\Phi\left(x, p_{T}\right)$.

The relations in (5.44) only involve collinear functions of twist two and those in (5.45) only collinear functions of twist three, corresponding to the respective order in the $1 / p$ expansion of the correlation function $\Phi\left(x, p_{T}\right)$. On the other hand, there are $p_{T}$-dependent functions of twist two and twist three in both (5.44) and (5.45). In other words, the twist of the collinear distributions and the $p_{T}$-dependent distributions in the high- $p_{T}$ limit need not be the same.

By power counting, the $p_{T}$-dependent distributions $f_{L}^{\perp}, g^{\perp}, e_{T}, e_{T}^{\perp}$ can receive a contribution from the $1 / p^{2}$ part of $\Phi\left(x, p_{T}\right)$, but explicit calculation at order $\alpha_{s}$ gives a zero result. This readily follows from our discussion above (5.44) because these distributions are $T$-odd. Their high- $p_{T}$ behavior therefore starts at order $\alpha_{s}^{2} / p^{2}$ and reads

$$
\begin{aligned}
x f_{L}^{\perp} & \sim \frac{1}{\boldsymbol{p}_{T}^{2}} \alpha_{s}^{2} \mathcal{F}\left[g_{1}\right], & x g^{\perp} & \sim \frac{1}{\boldsymbol{p}_{T}^{2}} \alpha_{s}^{2} \mathcal{F}\left[f_{1}\right], \\
x e_{T} & \sim \frac{1}{\boldsymbol{p}_{T}^{2}} \alpha_{s}^{2} \mathcal{F}\left[h_{1}\right], & x e_{T}^{\perp} & \sim \frac{1}{\boldsymbol{p}_{T}^{2}} \alpha_{s}^{2} \mathcal{F}\left[h_{1}\right] .
\end{aligned}
$$

There will also be contributions to these functions from $\Phi\left(x, p_{T}\right)$ order $\alpha_{s} / p^{4}$, where the necessary $T$-odd effects can come from lowest-order graphs with three- or four-parton correlation functions. By power counting in $1 / p$, these contributions are subleading compared with ones in (5.47), although they appear at lower order in the $\alpha_{s}$ expansion. We omit them in our subsequent discussion, but they can easily be restored.

Explicit calculation also reveals that neither $h_{1 T}^{\perp}\left(x, p_{T}\right)$ nor the combination $h_{T}\left(x, p_{T}\right)+$ $h_{T}^{\perp}\left(x, p_{T}\right)$ receives contributions at order $\alpha_{s}$ and $1 / p^{2}$, although this would be allowed by power counting. We shall not investigate the reason of this here, and simply write

$$
h_{1 T}^{\perp} \sim \frac{M^{2}}{\boldsymbol{p}_{T}^{4}} \alpha_{s}^{2} \mathcal{F}\left[h_{1}\right], \quad \quad x h_{T}+x h_{T}^{\perp} \sim \frac{1}{\boldsymbol{p}_{T}^{2}} \alpha_{s}^{2} \mathcal{F}\left[h_{1}\right] .
$$

Again there will also be power suppressed contributions at lower order in $\alpha_{s}$, which go like $\alpha_{s} / p^{6}$ for $h_{1 T}^{\perp}$ and like $\alpha_{s} / p^{4}$ for $h_{T}+h_{T}^{\perp}$. We caution that without a full calculation of the graphs with multi-parton correlators we cannot exclude that the contributions to the distributions given in (5.45) vanish when all terms are added up. A corresponding caveat applies to the $\alpha_{s}^{2}$ contributions in (5.47) and (5.48). For $\Phi\left(x, p_{T}\right)$ at order $\alpha_{s}$ and $1 / p^{2}$ we give complete and explicit results in section 8 . As for the $1 / p^{3}$ part of $\Phi\left(x, p_{T}\right)$, the explicit calculation in [6, 8] gives $f_{1 T}^{\perp}\left(x, p_{T}^{2}\right) \sim\left(M^{2} / \boldsymbol{p}_{T}^{4}\right) \alpha_{s} \mathcal{F}\left[G_{F}, \widetilde{G}_{F}\right]$, where $\mathcal{F}$ now 
denotes two-variable convolutions of the form

$$
\int \frac{d \hat{x}_{1}}{\hat{x}_{1}} \frac{d \hat{x}_{2}}{\hat{x}_{2}} K\left(\hat{x}_{1}, \hat{x}_{2}\right) G_{F}\left(\frac{x}{\hat{x}_{1}}, \frac{x}{\hat{x}_{2}}\right) .
$$

Given that $f_{1 T}^{\perp(1)[ \pm]}(x)=\mp \frac{\pi}{2} G_{F}(x, x)$, the structure of our result for $f_{1 T}^{\perp}\left(x, p_{T}^{2}\right)$ in 5.45 is hence consistent with the full calculation. ${ }^{3}$

At this point we briefly return to the question of ultraviolet divergences in collinear correlation functions, which we mentioned after (5.12). With the high- $p_{T}$ behavior given in (5.44) and (5.45) one explicitly sees that the integral $\int d^{2} \boldsymbol{p}_{T} \Phi\left(x, p_{T}\right)$ diverges logarithmically at high $p_{T}$, both for the twist-two and the twist-three part of $\Phi\left(x, p_{T}\right)$. The corresponding ultraviolet subtractions in the collinear correlator $\Phi(x)$ result in a logarithmic dependence on the subtraction scale $\mu$ for all distributions in (5.13). This dependence is described by DGLAP equations, whose evolution kernels are closely related to the kernels appearing in the convolutions of (5.44) and (5.45). With (5.45) one also finds that the integral $\int d^{2} \boldsymbol{p}_{T} p_{T}^{\alpha} \Phi_{2}\left(x, p_{T}\right)$ diverges logarithmically. This leads to DGLAP equations for the $\boldsymbol{p}_{T}^{2}$ moments of twist-two distributions in the parameterization (5.19) of $\Phi_{\partial}(x)$, which have been investigated in [57]. In contrast, the integral $\int d^{2} \boldsymbol{p}_{T} p_{T}^{\alpha} \Phi_{3}\left(x, p_{T}\right)$ diverges quadratically in $p_{T}$ according to (5.44) and (5.47). In a proper definition for $\boldsymbol{p}_{T}^{2}$-moments of twist-three distributions, such as $f^{\perp(1)}(x)$ or $g^{\perp(1)}(x)$, one would hence have to deal with power-like divergences.

In the dimensional analysis following (5.42) we have ignored that the hard-scattering kernels also depend on the regularization parameter $\zeta$, which is Lorentz invariant and has mass dimension two. In applications of low- $q_{T}$ factorization one needs $\zeta$ comparable to the large scale, as already mentioned in section 3.1, so that we can restrict our attention to $\zeta \gg p_{T}^{2}$. Terms in the hard-scattering kernels going with a positive power of $p_{T}^{2} / \zeta$ are then negligible. In contrast, terms with a positive power of $\zeta / p_{T}^{2}$ would lead to a faster $p_{T}^{2}$ falloff than derived in this section. They would correspond to power-like rapidity divergences in $\Phi\left(x, p_{T}\right)$. In the explicit calculations at order $1 / p^{2}$ in section 8 we will not encounter such terms, obtaining only a modification of the power-laws in (5.44) by $\operatorname{logarithms} \ln \left(\zeta / p_{T}^{2}\right)$. A corresponding statement holds for the calculation of $f_{1 T}^{\perp}\left(x, p_{T}^{2}\right)$ in [6, 8].

The high- $k_{T}$ behavior of the fragmentation correlator $\Delta\left(z, k_{T}\right)$ can be obtained in full analogy to the case of $\Phi\left(x, p_{T}\right)$. One can readily obtain results by crossing the hardscattering graphs calculated for the distribution functions, replacing $x \rightarrow 1 / z$ and $p_{T} \rightarrow k_{T}$. This gives

$$
\begin{aligned}
D_{1} & \sim \frac{1}{\boldsymbol{k}_{T}^{2}} \alpha_{s} \mathcal{F}\left[D_{1}\right], & \frac{D^{\perp}}{z} & \sim \frac{1}{\boldsymbol{k}_{T}^{2}} \alpha_{s} \mathcal{F}\left[D_{1}\right], \\
H_{1}^{\perp} & \sim \frac{M^{2}}{\boldsymbol{k}_{T}^{4}} \alpha_{s} \mathcal{F}\left[H_{1}^{\perp(1)}, \ldots\right], & \frac{G^{\perp}}{z} & \sim \frac{1}{\boldsymbol{k}_{T}^{2}} \alpha_{s}^{2} \mathcal{F}\left[D_{1}\right], \\
\frac{H}{z} & \sim \frac{1}{\boldsymbol{k}_{T}^{2}} \alpha_{s} \mathcal{F}\left[H_{1}^{\perp(1)}, \ldots\right], & \frac{E}{z} & \sim \frac{1}{\boldsymbol{k}_{T}^{2}} \alpha_{s} \mathcal{F}\left[\frac{E}{z}, \ldots\right] .
\end{aligned}
$$

\footnotetext{
${ }^{3}$ The relation between $f_{1 T}^{\perp(1)}$ and $G_{F}$ can be obtained by combining (5.19) in the present work with eq. (2) in 43] and eqs. (29), (40) in [13. Corresponding relations using different parameterizations have been given in [56] and [5].
} 
Compared with their analogs (5.46), the convolutions

$$
\mathcal{F}[D]=\frac{1}{z^{2}}\left[D^{a} \otimes K_{q}+D^{g} \otimes K_{g}\right]
$$

have an additional factor $1 / z^{2}$, which reflects the corresponding factor in (5.21).

\subsection{Results for structure functions}

Let us begin this section by recalling the expressions for SIDIS structure functions at low $q_{T}$ in terms of transverse-momentum-dependent distribution and fragmentation functions. Extending earlier work in [11, 12], the study [14 has given a complete set of results at leading and first subleading order in $1 / Q$, i.e., at twist-two and twist-three accuracy. A detailed investigation of color gauge invariance and the appropriate choice of gauge links has been given in [13]. The calculations just quoted take into account tree-level graphs, where gluons are restricted to be collinear to the target or to the observed hadron $h$ and only appear when they are attached to the distribution or fragmentation correlators (see figure 2 in [14]).

For a compact presentation of the results, we introduce the unit vector $\hat{\boldsymbol{h}}=-\boldsymbol{q}_{T} /\left|\boldsymbol{q}_{T}\right|$ and the transverse-momentum convolution

$$
\mathcal{C}[w f D]=\sum_{a} x e_{a}^{2} \int d^{2} \boldsymbol{p}_{T} d^{2} \boldsymbol{k}_{T} \delta^{(2)}\left(\boldsymbol{p}_{T}-\boldsymbol{k}_{T}+\boldsymbol{q}_{T}\right) w\left(\boldsymbol{p}_{T}, \boldsymbol{k}_{T}\right) f^{a}\left(x, p_{T}^{2}\right) D^{a}\left(z, k_{T}^{2}\right),
$$

where $w\left(\boldsymbol{p}_{T}, \boldsymbol{k}_{T}\right)$ is an arbitrary function and the sum runs over quarks and antiquarks. The results for the structure functions appearing in (2.3) then read 14]

$$
\begin{aligned}
F_{U U, T} & =\mathcal{C}\left[f_{1} D_{1}\right] \\
F_{U U, L} & =\mathcal{O}\left(\frac{M^{2}}{Q^{2}}, \frac{q_{T}^{2}}{Q^{2}}\right) \\
F_{U U}^{\cos \phi_{h}} & =\frac{2 M}{Q} \mathcal{C}\left[-\frac{\hat{\boldsymbol{h}} \cdot \boldsymbol{k}_{T}}{M_{h}}\left(x h H_{1}^{\perp}+\frac{M_{h}}{M} f_{1} \frac{\tilde{D}^{\perp}}{z}\right)-\frac{\hat{\boldsymbol{h}} \cdot \boldsymbol{p}_{T}}{M}\left(x f^{\perp} D_{1}+\frac{M_{h}}{M} h_{1}^{\perp} \frac{\tilde{H}}{z}\right)\right], \\
F_{U U}^{\cos 2 \phi_{h}} & =\mathcal{C}\left[-\frac{2\left(\hat{\boldsymbol{h}} \cdot \boldsymbol{k}_{T}\right)\left(\hat{\boldsymbol{h}} \cdot \boldsymbol{p}_{T}\right)-\boldsymbol{k}_{T} \cdot \boldsymbol{p}_{T}}{M M_{h}} h_{1}^{\perp} H_{1}^{\perp}\right] \\
F_{L U}^{\sin \phi_{h}} & =\frac{2 M}{Q} \mathcal{C}\left[-\frac{\hat{\boldsymbol{h}} \cdot \boldsymbol{k}_{T}}{M_{h}}\left(x e H_{1}^{\perp}+\frac{M_{h}}{M} f_{1} \frac{\tilde{G}^{\perp}}{z}\right)+\frac{\hat{\boldsymbol{h}} \cdot \boldsymbol{p}_{T}}{M}\left(x g^{\perp} D_{1}+\frac{M_{h}}{M} h_{1}^{\perp} \frac{\tilde{E}}{z}\right)\right] \\
F_{U L}^{\sin \phi_{h}} & =\frac{2 M}{Q} \mathcal{C}\left[-\frac{\hat{\boldsymbol{h}} \cdot \boldsymbol{k}_{T}}{M_{h}}\left(x h_{L} H_{1}^{\perp}+\frac{M_{h}}{M} g_{1 L} \frac{\tilde{G}^{\perp}}{z}\right)+\frac{\hat{\boldsymbol{h}} \cdot \boldsymbol{p}_{T}}{M}\left(x f_{L}^{\perp} D_{1}-\frac{M_{h}}{M} h_{1 L}^{\perp} \frac{\tilde{H}}{z}\right)\right] \\
F_{U L}^{\sin 2 \phi_{h}} & =\mathcal{C}\left[-\frac{\left.2\left(\hat{\boldsymbol{h}} \cdot \boldsymbol{k}_{T}\right)\left(\hat{\boldsymbol{h}} \cdot \boldsymbol{p}_{T}\right)-\boldsymbol{k}_{T} \cdot \boldsymbol{p}_{T} h_{1 L}^{\perp} H_{1}^{\perp}\right],}{M M_{h}}\right. \\
F_{L L} & =\mathcal{C}\left[g_{1 L} D_{1}\right], \\
F_{L L}^{\cos \phi_{h}} & =\frac{2 M}{Q} \mathcal{C}\left[\frac{\hat{\boldsymbol{h}} \cdot \boldsymbol{k}_{T}}{M_{h}}\left(x e_{L} H_{1}^{\perp}-\frac{M_{h}}{M} g_{1 L} \frac{\tilde{D}^{\perp}}{z}\right)-\frac{\hat{\boldsymbol{h}} \cdot \boldsymbol{p}_{T}}{M}\left(x g_{L}^{\perp} D_{1}+\frac{M_{h}}{M} h_{1 L}^{\perp} \frac{\tilde{E}}{z}\right)\right],
\end{aligned}
$$




$$
\begin{aligned}
& F_{U T, T}^{\sin \left(\phi_{h}-\phi_{S}\right)}=\mathcal{C}\left[-\frac{\hat{\boldsymbol{h}} \cdot \boldsymbol{p}_{T}}{M} f_{1 T}^{\perp} D_{1}\right] \\
& F_{U T, L}^{\sin \left(\phi_{h}-\phi_{S}\right)}=\mathcal{O}\left(\frac{M^{2}}{Q^{2}}, \frac{q_{T}^{2}}{Q^{2}}\right) \\
& F_{U T}^{\sin \left(\phi_{h}+\phi_{S}\right)}=\mathcal{C}\left[-\frac{\hat{\boldsymbol{h}} \cdot \boldsymbol{k}_{T}}{M_{h}} h_{1} H_{1}^{\perp}\right] \text {, } \\
& F_{U T}^{\sin \left(3 \phi_{h}-\phi_{S}\right)}=\mathcal{C}\left[\frac{2\left(\hat{\boldsymbol{h}} \cdot \boldsymbol{p}_{T}\right)\left(\boldsymbol{p}_{T} \cdot \boldsymbol{k}_{T}\right)+\boldsymbol{p}_{T}^{2}\left(\hat{\boldsymbol{h}} \cdot \boldsymbol{k}_{T}\right)-4\left(\hat{\boldsymbol{h}} \cdot \boldsymbol{p}_{T}\right)^{2}\left(\hat{\boldsymbol{h}} \cdot \boldsymbol{k}_{T}\right)}{2 M^{2} M_{h}} h_{1 T}^{\perp} H_{1}^{\perp}\right], \\
& F_{U T}^{\sin \phi_{S}}=\frac{2 M}{Q} \mathcal{C}\left\{\left(x f_{T} D_{1}-\frac{M_{h}}{M} h_{1} \frac{\tilde{H}}{z}\right)\right. \\
& \left.-\frac{\boldsymbol{k}_{T} \cdot \boldsymbol{p}_{T}}{2 M M_{h}}\left[\left(x h_{T} H_{1}^{\perp}+\frac{M_{h}}{M} g_{1 T} \frac{\tilde{G}^{\perp}}{z}\right)-\left(x h_{T}^{\perp} H_{1}^{\perp}-\frac{M_{h}}{M} f_{1 T}^{\perp} \frac{\tilde{D}^{\perp}}{z}\right)\right]\right\}, \\
& F_{U T}^{\sin \left(2 \phi_{h}-\phi_{S}\right)}=\frac{2 M}{Q} \mathcal{C}\left\{\frac{2\left(\hat{\boldsymbol{h}} \cdot \boldsymbol{p}_{T}\right)^{2}-\boldsymbol{p}_{T}^{2}}{2 M^{2}}\left(x f_{T}^{\perp} D_{1}-\frac{M_{h}}{M} h_{1 T}^{\perp} \frac{\tilde{H}}{z}\right)\right. \\
& -\frac{2\left(\hat{\boldsymbol{h}} \cdot \boldsymbol{k}_{T}\right)\left(\hat{\boldsymbol{h}} \cdot \boldsymbol{p}_{T}\right)-\boldsymbol{k}_{T} \cdot \boldsymbol{p}_{T}}{2 M M_{h}}\left[\left(x h_{T} H_{1}^{\perp}+\frac{M_{h}}{M} g_{1 T} \frac{\tilde{G}^{\perp}}{z}\right)\right. \\
& \left.\left.+\left(x h_{T}^{\perp} H_{1}^{\perp}-\frac{M_{h}}{M} f_{1 T}^{\perp} \frac{\tilde{D}^{\perp}}{z}\right)\right]\right\}, \\
& F_{L T}^{\cos \left(\phi_{h}-\phi_{S}\right)}=\mathcal{C}\left[\frac{\hat{\boldsymbol{h}} \cdot \boldsymbol{p}_{T}}{M} g_{1 T} D_{1}\right], \\
& F_{L T}^{\cos \phi_{S}}=\frac{2 M}{Q} \mathcal{C}\left\{-\left(x g_{T} D_{1}+\frac{M_{h}}{M} h_{1} \frac{\tilde{E}}{z}\right)\right. \\
& \left.+\frac{\boldsymbol{k}_{T} \cdot \boldsymbol{p}_{T}}{2 M M_{h}}\left[\left(x e_{T} H_{1}^{\perp}-\frac{M_{h}}{M} g_{1 T} \frac{\tilde{D}^{\perp}}{z}\right)+\left(x e_{T}^{\perp} H_{1}^{\perp}+\frac{M_{h}}{M} f_{1 T}^{\perp} \frac{\tilde{G}^{\perp}}{z}\right)\right]\right\}, \\
& F_{L T}^{\cos \left(2 \phi_{h}-\phi_{S}\right)}=\frac{2 M}{Q} \mathcal{C}\left\{-\frac{2\left(\hat{\boldsymbol{h}} \cdot \boldsymbol{p}_{T}\right)^{2}-\boldsymbol{p}_{T}^{2}}{2 M^{2}}\left(x g_{T}^{\perp} D_{1}+\frac{M_{h}}{M} h_{1 T}^{\perp} \frac{\tilde{E}}{z}\right)\right. \\
& +\frac{2\left(\hat{\boldsymbol{h}} \cdot \boldsymbol{k}_{T}\right)\left(\hat{\boldsymbol{h}} \cdot \boldsymbol{p}_{T}\right)-\boldsymbol{k}_{T} \cdot \boldsymbol{p}_{T}}{2 M M_{h}}\left[\left(x e_{T} H_{1}^{\perp}-\frac{M_{h}}{M} g_{1 T} \frac{\tilde{D}^{\perp}}{z}\right)\right. \\
& \left.\left.-\left(x e_{T}^{\perp} H_{1}^{\perp}+\frac{M_{h}}{M} f_{1 T}^{\perp} \frac{\tilde{G}^{\perp}}{z}\right)\right]\right\} .
\end{aligned}
$$

In the entries for $F_{U U, L}$ and $F_{U T, L}^{\sin \left(\phi_{h}-\phi_{S}\right)}$ we have indicated that these structure functions come out to be zero when the calculation includes only terms up to order $1 / Q$. The fragmentation functions with a tilde are given by

$$
\begin{aligned}
& \frac{\tilde{D}^{\perp}}{z}=\frac{D^{\perp}}{z}-D_{1}, \\
& \frac{\tilde{G}^{\perp}}{z}=\frac{G^{\perp}}{z}-\frac{m}{M_{h}} H_{1}^{\perp},
\end{aligned}
$$




$$
\begin{aligned}
\frac{\tilde{E}}{z} & =\frac{E}{z}-\frac{m}{M_{h}} D_{1}, \\
\frac{\tilde{H}}{z} & =\frac{H}{z}+\frac{\boldsymbol{k}_{T}^{2}}{M_{h}^{2}} H_{1}^{\perp} .
\end{aligned}
$$

Using (5.50) and neglecting the small contributions proportional to the quark mass $m$, we readily see that the behavior for $k_{T} \gg M$ is the same for corresponding functions with and without a tilde.

The tree-level calculations in [11, 13, 14] do not take into account soft gluon exchange or virtual corrections involving hard loops, so that the soft and hard factors we encountered in (3.3) and (3.22) do not appear in the convolution (5.52). Detailed investigations of factorization for SIDIS with measured $q_{T}$ have recently been given in [26, 36] and [50, 27], extending the seminal work of Collins and Soper [24]. The factorization formulae discussed in these papers have the form (3.22) and are valid at all orders in $\alpha_{s}$ but restricted to the leading order in $1 / Q$. Although a number of subtle issues remain to be fully clarified [27], we will use (3.22) in the following. Since we aim at deriving expressions at lowest nonvanishing order in $\alpha_{s}$, we can neglect the hard factor $|H|^{2}=1+O\left(\alpha_{s}\right)$. The convolution in (5.52) should then be extended to

$$
\begin{gathered}
\mathcal{C}[w f D]=\sum_{a} x e_{a}^{2} \int d^{2} \boldsymbol{p}_{T} d^{2} \boldsymbol{k}_{T} d^{2} \boldsymbol{l}_{T} \delta^{(2)}\left(\boldsymbol{p}_{T}-\boldsymbol{k}_{T}+\boldsymbol{l}_{T}+\boldsymbol{q}_{T}\right) \\
\times w\left(\boldsymbol{p}_{T}, \boldsymbol{k}_{T}\right) f^{a}\left(x, p_{T}^{2}\right) D^{a}\left(z, k_{T}^{2}\right) U\left(l_{T}^{2}\right) .
\end{gathered}
$$

At high transverse momentum $l_{T} \gg M$ the soft factor behaves as $U\left(l_{T}^{2}\right) \sim \alpha_{s} / \boldsymbol{l}_{T}^{2}$, with a coefficient we shall give in (8.51) below. Our normalization convention is

$$
\int d^{2} \boldsymbol{l}_{T} U\left(l_{T}^{2}\right)=1+O\left(\alpha_{s}\right)
$$

where it is understood that the integral must be suitably regularized at large $l_{T}$.

Whether Collins-Soper factorization can be extended to structure functions that are of order $1 / Q$ is not known. We note that the study of color gauge invariance in 13] was limited to $q_{T}$-integrated observables in this case, and that a problem with twist-three factorization has been found in a spectator model calculation [15]. In the following we adopt the working hypothesis that the twist-two factorization formula can simply be taken over at twist-three accuracy, using the convolution (5.75) also for evaluating the high- $q_{T}$ behavior of the $1 / Q$ suppressed structure functions in (5.53) to (5.70). We will return to this point at the end of section 8.3 .

We now show how to calculate the high- $q_{T}$ behavior of the convolution (5.75). At order $\alpha_{s}$, only one of the factors $f\left(x, p_{T}^{2}\right), D\left(z, k_{T}^{2}\right), U\left(l_{T}^{2}\right)$ can be taken at high transverse momentum. Let us first consider the simple case where $w\left(\boldsymbol{p}_{T}, \boldsymbol{k}_{T}\right)=1$. In the region where $p_{T}$ is large, we use the $\delta$ function in (5.75) to perform the $p_{T}$ integral and approximate $\boldsymbol{p}_{T}=\boldsymbol{k}_{T}-\boldsymbol{l}_{T}-\boldsymbol{q}_{T} \approx-\boldsymbol{q}_{T}$ in $f\left(x, p_{T}^{2}\right)$. The remaining integrals over $k_{T}$ and $l_{T}$ can then be carried out independently. According to (5.21) and our discussion after (5.16), the integral over $k_{T}$ gives a collinear fragmentation function, up to $\alpha_{s}$-corrections that can be neglected 
to our accuracy. Likewise, the integral over $l_{T}$ gives unity up to $\alpha_{s}$-corrections according to (5.76). Since we are considering the region where $k_{T}$ and $l_{T}$ are small compared with $q_{T}$ the integrals over these momenta should be suitably cut off, as is required for (5.21) and (5.76) to make sense. Repeating these arguments for the cases where $k_{T}$ or $l_{T}$ are large, we obtain

$$
\begin{aligned}
& \int d^{2} \boldsymbol{p}_{T} d^{2} \boldsymbol{k}_{T} d^{2} \boldsymbol{l}_{T} \delta^{(2)}\left(\boldsymbol{p}_{T}-\boldsymbol{k}_{T}+\boldsymbol{l}_{T}+\boldsymbol{q}_{T}\right) f\left(x, p_{T}^{2}\right) D\left(z, k_{T}^{2}\right) U\left(l_{T}^{2}\right) \\
& \approx f\left(x, q_{T}^{2}\right) \frac{D(z)}{z^{2}}+f(x) D\left(z, q_{T}^{2}\right)+f(x) \frac{D(z)}{z^{2}} U\left(q_{T}^{2}\right) .
\end{aligned}
$$

For nontrivial functions $w\left(\boldsymbol{p}_{T}, \boldsymbol{k}_{T}\right)$ the calculation is slightly more involved. Instead of approximating e.g. $\boldsymbol{p}_{T}=\boldsymbol{k}_{T}-\boldsymbol{l}_{T}-\boldsymbol{q}_{T} \approx-\boldsymbol{q}_{T}$, we need to Taylor expand the functions of $\boldsymbol{p}_{T}$ around $-\boldsymbol{q}_{T}$. We take as an example the convolution $\mathcal{C}\left[\left(\boldsymbol{k}_{T} \cdot \boldsymbol{p}_{T}\right) h_{1}^{\perp} H_{1}^{\perp}\right]$ appearing in $F_{U U}^{\cos 2 \phi_{h}}$ and consider the region where $p_{T}$ is large. We perform the integral over $p_{T}$ using the $\delta$ function and obtain

$$
\begin{aligned}
\int d^{2} \boldsymbol{k}_{T} d^{2} \boldsymbol{l}_{T} H_{1}^{\perp}\left(z, k_{T}^{2}\right) U\left(l_{T}^{2}\right)\left(\boldsymbol{k}_{T}^{2}-\boldsymbol{k}_{T} \cdot \boldsymbol{l}_{T}-\boldsymbol{k}_{T} \cdot \boldsymbol{q}_{T}\right) h_{1}^{\perp}\left(x,\left(\boldsymbol{k}_{T}-\boldsymbol{l}_{T}-\boldsymbol{q}_{T}\right)^{2}\right) \\
\approx \int d^{2} \boldsymbol{k}_{T} d^{2} \boldsymbol{l}_{T} H_{1}^{\perp}\left(z, k_{T}^{2}\right) U\left(l_{T}^{2}\right) \\
\quad \times\left(\boldsymbol{k}_{T}^{2}-\boldsymbol{k}_{T} \cdot \boldsymbol{l}_{T}-\boldsymbol{k}_{T} \cdot \boldsymbol{q}_{T}\right)\left[h_{1}^{\perp}\left(x, q_{T}^{2}\right)-2\left(\boldsymbol{k}_{T} \cdot \boldsymbol{q}_{T}-\boldsymbol{l}_{T} \cdot \boldsymbol{q}_{T}\right) \frac{\partial}{\partial \boldsymbol{q}_{T}^{2}} h_{1}^{\perp}\left(x, q_{T}^{2}\right)\right]+\cdots \\
\approx \int d^{2} \boldsymbol{k}_{T} H_{1}^{\perp}\left(z, k_{T}^{2}\right)\left[\boldsymbol{k}_{T}^{2} h_{1}^{\perp}\left(x, q_{T}^{2}\right)+2\left(\boldsymbol{k}_{T} \cdot \boldsymbol{q}_{T}\right)^{2} \frac{\partial}{\partial \boldsymbol{q}_{T}^{2}} h_{1}^{\perp}\left(x, q_{T}^{2}\right)\right]+\cdots \\
=2 M_{h}^{2} \frac{H_{1}^{\perp(1)}(z)}{z^{2}}\left[h_{1}^{\perp}\left(x, q_{T}^{2}\right)+\boldsymbol{q}_{T}^{2} \frac{\partial}{\partial \boldsymbol{q}_{T}^{2}} h_{1}^{\perp}\left(x, q_{T}^{2}\right)\right]+\cdots
\end{aligned}
$$

where both terms in square brackets behave as $1 / q_{T}^{4}$. The ... represent contributions from the regions where $k_{T}$ or $l_{T}$ is large, which are of the same order in $1 / q_{T}$.

As we see in (5.47), (5.48), and (5.50), the leading power behavior of some distribution or fragmentation functions comes with a factor $\alpha_{s}^{2}$. At this order, one must also take into account regions of integration in (5.75) where two out of the three momenta $p_{T}, k_{T}, l_{T}$ are large, but it turns out that these do not contribute to the $\alpha_{s}^{2}$ terms given in the following. Using the high-transverse-momentum behavior in (5.44) to (5.48) and (5.50), we obtain

$$
\begin{aligned}
F_{U U, T} & \sim \frac{1}{q_{T}^{2}} \alpha_{s} \mathcal{F}\left[f_{1} D_{1}\right], \\
F_{U U}^{\cos \phi_{h}} & \sim \frac{1}{Q q_{T}} \alpha_{s} \mathcal{F}\left[f_{1} D_{1}\right], \\
F_{U U}^{\cos 2 \phi_{h}} & \sim \frac{M^{2}}{q_{T}^{4}} \alpha_{s} \mathcal{F}\left[h_{1}^{\perp(1)} H_{1}^{\perp(1)}, \ldots\right], \\
F_{L U}^{\sin \phi_{h}} & \sim \frac{1}{Q q_{T}} \alpha_{s}^{2} \mathcal{F}\left[f_{1} D_{1}\right], \\
F_{U L}^{\sin \phi_{h}} & \sim \frac{1}{Q q_{T}} \alpha_{s}^{2} \mathcal{F}\left[g_{1} D_{1}\right],
\end{aligned}
$$




$$
\begin{aligned}
F_{U L}^{\sin 2 \phi_{h}} & \sim \frac{M^{2}}{q_{T}^{4}} \alpha_{s} \mathcal{F}\left[h_{1 L}^{\perp(1)} H_{1}^{\perp(1)}, \ldots\right], \\
F_{L L} & \sim \frac{1}{q_{T}^{2}} \alpha_{s} \mathcal{F}\left[g_{1} D_{1}\right], \\
F_{L L}^{\cos \phi_{h}} & \sim \frac{1}{Q q_{T}} \alpha_{s} \mathcal{F}\left[g_{1} D_{1}\right], \\
F_{U T, T}^{\sin \left(\phi_{h}-\phi_{S}\right)} & \sim \frac{M}{q_{T}^{3}} \alpha_{s} \mathcal{F}\left[f_{1 T}^{\perp(1)} D_{1}, \ldots\right], \\
F_{U T}^{\sin \left(\phi_{h}+\phi_{S}\right)} & \sim \frac{M}{q_{T}^{3}} \alpha_{s} \mathcal{F}\left[h_{1} H_{1}^{\perp(1)}, \ldots\right], \\
F_{U T}^{\sin \left(3 \phi_{h}-\phi_{S}\right)} & \sim \frac{M}{q_{T}^{3}} \alpha_{s}^{2} \mathcal{F}\left[h_{1} H_{1}^{\perp(1)}, \ldots\right], \\
F_{U T}^{\sin \phi_{S}} & \sim \frac{M}{Q q_{T}^{2}} \alpha_{s} \mathcal{F}\left[f_{1 T}^{\perp(1)} D_{1}, h_{1} H_{1}^{\perp(1)}, \ldots\right], \\
F_{U T}^{\sin \left(2 \phi_{h}-\phi_{S}\right)} & \sim \frac{M}{Q q_{T}^{2}} \alpha_{s} \mathcal{F}\left[f_{1 T}^{\perp(1)} D_{1}, \ldots\right], \\
F_{L T}^{\cos \left(\phi_{h}-\phi_{S}\right)} & \sim \frac{M}{q_{T}^{3}} \alpha_{s} \mathcal{F}\left[g_{1 T}^{(1)} D_{1}, \ldots\right], \\
F_{L T}^{\cos \phi_{S}} & \sim \frac{M}{Q q_{T}^{2}} \alpha_{s} \mathcal{F}\left[g_{1 T}^{(1)} D_{1}, h_{1} \frac{E}{z}, \ldots\right] \\
F_{L T}^{\cos \left(2 \phi_{h}-\phi_{S}\right)} & \sim \frac{M}{Q q_{T}^{2}} \alpha_{s} \mathcal{F}\left[g_{1 T}^{(1)} D_{1}, \ldots\right] .
\end{aligned}
$$

Here either the parton distributions or the fragmentation functions are convoluted with kernels $K_{i}$ or $L_{i}$ :

$$
\mathcal{F}[f D]=\frac{1}{z^{2}} \sum_{a, i} e_{a}^{2}\left[\left(K_{i} \otimes f^{i}\right)(x) D^{a}(z)+f^{a}(x)\left(D^{i} \otimes L_{i}\right)(z)\right],
$$

where the sum runs over quarks and antiquarks for $a$ and over quarks, antiquarks and gluons for $i$. As we will see in section 8, these kernels contain logarithms of $Q / q_{T}$. Their origin is the dependence of $f_{1}\left(x, p_{T}^{2}\right)$ or $D_{1}\left(z, k_{T}^{2}\right)$ on $\zeta$ or $\zeta_{h}$, which we tacitly omitted in (5.75). When resummed to all orders in $\alpha_{s}$ in the way we sketched in section 3, these logarithms can lead to a substantial modification of the power laws in (5.79) to (5.94). A numerical study of these effects on azimuthal asymmetries in Drell-Yan production has been performed in [58].

We note that for the $1 / Q$ suppressed structure functions in (5.79) to (5.94), contributions from $U\left(l_{T}^{2}\right)$ taken at $\boldsymbol{l}_{T} \approx-\boldsymbol{q}_{T}$ are power suppressed or have the same power behavior as contributions where either $\boldsymbol{p}_{T} \approx-\boldsymbol{q}_{T}$ or $\boldsymbol{k}_{T} \approx \boldsymbol{q}_{T}$. For these structure functions, the power behavior at high $q_{T}$ hence remains the same if we simply ignore the soft factor and work with the tree-level convolution (5.52) instead of (5.75).

\section{Comparing results at intermediate $\boldsymbol{q}_{T}$}

We can now compare the results for the region $M \ll q_{T} \ll Q$ obtained in the low- $q_{T}$ calculation of the previous section with those obtained in the high- $q_{T}$ calculation. As we 
mentioned in section 4 , not all structure functions have been calculated in the high- $q_{T}$ picture. For the cases where results are available, we find

$$
\begin{aligned}
F_{U U, T} & \sim \frac{1}{q_{T}^{2}} \alpha_{s} \mathcal{F}\left[f_{1} D_{1}\right], \\
F_{U U, L} & \sim \frac{1}{Q^{2}} \alpha_{s} \mathcal{F}\left[f_{1} D_{1}\right], \\
F_{U U}^{\cos \phi_{h}} & \sim \frac{1}{Q q_{T}} \alpha_{s} \mathcal{F}\left[f_{1} D_{1}\right], \\
F_{U U}^{\cos 2 \phi_{h}} & \sim \frac{1}{Q^{2}} \alpha_{s} \mathcal{F}\left[f_{1} D_{1}\right], \\
F_{L U}^{\sin \phi_{S}} & \sim \frac{1}{Q q_{T}} \alpha_{s}^{2} \mathcal{F}\left[f_{1} D_{1}\right], \\
F_{L L} & \sim \frac{1}{q_{T}^{2}} \alpha_{s} \mathcal{F}\left[g_{1} D_{1}\right], \\
F_{L L}^{\cos \phi_{h}} & \sim \frac{1}{Q q_{T}} \alpha_{s} \mathcal{F}\left[g_{1} D_{1}\right], \\
F_{U T, T}^{\sin \left(\phi_{h}-\phi_{S}\right)} & \sim \frac{M}{q_{T}^{3}} \alpha_{s} \mathcal{F}\left[G_{F} D_{1}, \widetilde{G}_{F} D_{1}\right], \\
F_{U T, L}^{\sin \left(\phi_{h}-\phi_{S}\right)} & \sim \frac{M}{Q^{2} q_{T}} \alpha_{s} \mathcal{F}\left[G_{F} D_{1}\right], \\
F_{U T}^{\sin \left(\phi_{h}+\phi_{S}\right)} & \sim \frac{M}{q_{T}^{3}} \alpha_{s} \mathcal{F}\left[h_{1} \widehat{E}_{F}\right], \\
F_{U T}^{\sin \left(3 \phi_{h}-\phi_{S}\right)} & \sim \frac{M}{Q^{2} q_{T}} \alpha_{s} \mathcal{F}\left[G_{F} D_{1}, \widetilde{G}_{F} D_{1}\right], \\
F_{U T}^{\sin \left(2 \phi_{h}-\phi_{S}\right)} & \sim \frac{M}{Q q_{T}^{2}} \alpha_{s} \mathcal{F}\left[G_{F} D_{1}, \widetilde{G}_{F} D_{1}\right] . \\
F_{U T}^{\sin \phi_{S}} & \sim \frac{M}{Q q_{T}^{2}} \alpha_{s} \mathcal{F}\left[G_{F} D_{1}, \widetilde{G}_{F} D_{1}, h_{1} \widehat{E}_{F}\right],
\end{aligned}
$$

The symbol $\mathcal{F}$ in (6.1) to (6.7) has the same meaning as in (5.95), whereas for the terms involving the three-parton correlation functions $G_{F}$ and $\widetilde{G}_{F}$ we have

$$
\mathcal{F}[G D]=\frac{1}{z^{2}} \sum_{a, i} e_{a}^{2}\left[\left(K_{i} \otimes G^{i}\right)(x) D^{a}(z)+G^{a}(x, x)\left(D^{i} \otimes L_{i}\right)(z)\right],
$$

where the two-variable convolution $\left(K_{i} \otimes G^{i}\right)(x)$ is of the form (5.49). The terms involving the three-parton fragmentation function $\widehat{E}_{F}$ in (6.10) and (6.12) are defined in analogy to $(6.14)$.

The results in (6.1) to (6.4) and (6.6) to (6.7) are directly taken from our expressions (4.23) to (4.28), whereas the result for $F_{L U}^{\mathrm{sin} \phi_{h}}$ in (6.5) has been extracted from the calculation in 45. The form of the $F_{U T}$ structure functions in (6.8) to (6.13) can be obtained by taking the limit $q_{T} \ll Q$ of the results of Eguchi et al. [43, 44], with the caveats discussed in section 4 . We note that the results of 443, 44, also contain terms involving the product $h_{1} \widehat{E}_{F}$ in $F_{U T, T}^{\sin \left(\phi_{h}-\phi_{S}\right)}$, as well as terms involving $G_{F} D_{1}$ or $\widetilde{G}_{F} D_{1}$ in 
$F_{U T}^{\sin \left(\phi_{h}+\phi_{S}\right)}$. However, these contributions behave like $M /\left(Q^{2} q_{T}\right)$ for $q_{T} \ll Q$ and are thus power suppressed compared with the terms given in (6.8) and (6.10).

Let us first discuss the unpolarized structure functions. Comparing the high- $q_{T}$ results (6.1) to (6.4) with the low- $q_{T}$ results (5.79) to (5.81), we find that at intermediate $q_{T}$ the power behavior of both $F_{U U, T}$ and $F_{U U}^{\cos \phi_{h}}$ agrees in the two calculations. We shall see in section 8.3 that in the case of $F_{U U, T}$ this agreement extends to the explicit expression of the structure function at order $\alpha_{s}$. By contrast, the leading power behavior obtained for $F_{U U}^{\cos 2 \phi_{h}}$ in the intermediate region is not the same in the low- and the high- $q_{T}$ calculations. In fact, the two results (6.4) and (5.81) describe two different physical mechanisms, since the low- $q_{T}$ calculation involves chiral-odd distribution and fragmentation functions, whereas the high- $q_{T}$ calculation involves chiral-even ones. Finally, the longitudinal structure function $F_{U U, L}$ only appears at order $1 / Q^{2}$ in the low- $q_{T}$ calculation and is hence beyond the accuracy of the results given in section 5.4. We remark that it is far from clear whether small- $q_{T}$ factorization still holds at twist-four level, given that even the twist-three case is not fully understood.

At this point we wish to discuss the calculation of the unpolarized structure functions at low transverse momentum in the parton model [59], where intrinsic transverse momentum is included in distribution and fragmentation functions and the kinematics is taken such that the quarks in the parton-level subprocess $\gamma^{*} q \rightarrow q$ are on shell. Using eqs. (4) and (32) of [59] and expanding in $1 / Q$, we obtain $F_{U U, T}=\mathcal{C}\left[f_{1} D_{1}\right]$ as in (5.53), whereas up to relative corrections in $1 / Q$ the other unpolarized structure functions read

$$
\begin{aligned}
F_{U U}^{\cos \phi_{h}} & =-\frac{2 M}{Q} \mathcal{C}\left[\frac{\hat{\boldsymbol{h}} \cdot \boldsymbol{p}_{T}}{M} f_{1} D_{1}\right], \\
F_{U U}^{\cos 2 \phi_{h}} & =\frac{4 M^{2}}{Q^{2}} \mathcal{C}\left[\frac{2\left(\hat{\boldsymbol{h}} \cdot \boldsymbol{p}_{T}\right)^{2}-\boldsymbol{p}_{T}^{2}}{2 M^{2}} f_{1} D_{1}\right], \quad F_{U U, L}=\frac{4 M^{2}}{Q^{2}} \mathcal{C}\left[\frac{\boldsymbol{p}_{T}^{2}}{M^{2}} f_{1} D_{1}\right],
\end{aligned}
$$

with the tree-level convolution $\mathcal{C}$ defined in (5.52). The modulations in $\cos \phi_{h}$ and $\cos 2 \phi_{h}$ obtained in this calculation are often referred to as Cahn effect [2, 3]. Taking the limit $q_{T} \gg M$ of the expressions in (6.15) we find the same power behavior as in the high- $q_{T}$ expressions (6.2) to (6.4). However, the high- $q_{T}$ behavior of (6.15) comes only from the high- $p_{T}$ tail of $f_{1}$ but not from the high- $k_{T}$ tail of $D_{1}$. It hence only involves terms of the form $\left(K_{i} \otimes f_{1}^{i}\right) D_{1}^{a}$, with the same kernels $K_{i}$ for $F_{U U}^{\cos \phi_{h}}, F_{U U}^{\cos 2 \phi_{h}}$, and $F_{U U, L}$. This readily implies that at intermediate $q_{T}$ the parton-model results (6.15) do not match with the explicit results $(4.24)$ to 4.26 ) of the high- $q_{T}$ calculation.

We remark that the high- $q_{T}$ limit of the full twist-three result (5.55) for $F_{U U}^{\cos \phi_{h}}$ comes from the chiral-even terms

$$
-\frac{2 M}{Q} \mathcal{C}\left[\frac{\hat{\boldsymbol{h}} \cdot \boldsymbol{k}_{T}}{M} f_{1} \frac{\tilde{D}^{\perp}}{z}+\frac{\hat{\boldsymbol{h}} \cdot \boldsymbol{p}_{T}}{M} x f^{\perp} D_{1}\right] .
$$

As observed in [14, this coincides with the parton model result 6.15) if one makes the Wandzura-Wilczek approximation, i.e., if one sets to zero the functions $\tilde{D}^{\perp}=D^{\perp}-z D_{1}$ and $x \tilde{f}^{\perp}=x f^{\perp}-f_{1}$, which are related to quark-gluon-quark correlation functions by the equation of motion for the quark field. We will see in section 8 that for $q_{T} \gg M$ these 
functions are in fact not negligible compared with $D_{1}$ and $f_{1}$, so that the approximations leading to 6.15) are not adequate in this limit. In a similar way, one may understand the parton model results for $F_{U U, L}$ and $F_{U U}^{\cos 2 \phi_{h}}$ as part of the (unknown) complete twist-four expression in a low- $q_{T}$ calculation. They have the correct power behavior to match the results (4.24) and (4.26) of the high- $q_{T}$ calculation, but do not reproduce all terms in these results. We note that $F_{U U}^{\cos 2 \phi_{h}}$ has the form (1.6) discussed in the introduction. The term with coefficient $l_{2,4}$ is given by the low- $q_{T}$ result (5.81), the term with $h_{2,4}$ by the high- $q_{T}$ expression (4.26), whereas the parton-model result (6.15) contributes to the subleading term $l_{4,2}$ in the low- $q_{T}$ power counting scheme.

Several phenomenological analyses, for instance those in 60-64, have used the parton model expressions for the unpolarized structure functions together with the high$q_{T}$ results $(4.23)$ to (4.26). We point out that in these papers a Gaussian behavior $f_{1}\left(x, p_{T}^{2}\right) \propto \exp \left[-a p_{T}^{2}\right]$ and $D_{1}\left(z, k_{T}^{2}\right) \propto \exp \left[-A k_{T}^{2}\right]$ is assumed for the distribution and fragmentation functions appearing in the parton model calculation. Such an approach differs from the one taken in the present work, where the power-law behavior of $f_{1}\left(x, p_{T}^{2}\right)$ and $D_{1}\left(z, k_{T}^{2}\right)$ at large transverse momentum is retained and explicitly calculated using perturbation theory.

Turning now to polarized observables, we find that the structure functions $F_{L L}$ and $F_{L L}^{\cos \phi_{h}}$ have the same power behavior in the high- and low- $q_{T}$ calculations, as do their unpolarized counterparts $F_{U U, T}$ and $F_{U U}^{\cos \phi_{h}}$. As in the unpolarized case, we will see in section 8.3 that $F_{L L}$ matches exactly at order $\alpha_{s}$ in the two calculations. Our low- $q_{T}$ result (5.82) for the $T$-odd structure function $F_{L U}^{\sin \phi_{S}}$ has the correct structure to match the limit (6.5) of the calculation at high $q_{T}$ and order $\alpha_{s}^{2}$ in [45]. One may expect that our low- $q_{T}$ result (5.83) for $F_{U L}^{\sin \phi_{S}}$ would also match with a high- $q_{T}$ calculation at the same order, but were are not aware of such a calculation in the literature.

For transverse polarization observables we compare eqs. (6.8) to 6.13) with (5.87) to (5.91) and see that four out of six structure functions have a matching power behavior, namely $F_{U T, T}^{\sin \left(\phi_{h}-\phi_{S}\right)}, F_{U T}^{\sin \left(\phi_{h}+\phi_{S}\right)}, F_{U T}^{\sin \phi_{S}}$, and $F_{U T}^{\sin \left(2 \phi_{h}-\phi_{S}\right)}$. The distribution and fragmentation functions appearing in the respective results are compatible as well, given that $f_{1 T}^{\perp(1)}$ is related with $G_{F}$ and $H_{1}^{\perp(1)}$ with $\widehat{E}_{F}$. As already mentioned in section 3.3, the explicit low- and high- $q_{T}$ calculations of $F_{U T, T}^{\sin \left(\phi_{h}-\phi_{S}\right)}$ in $\left[7, \&\right.$ found exact matching at order $\alpha_{s}$ for this observable. Looking at the remaining two $F_{U T}$ structure functions, we see that $F_{U T, L}^{\sin \left(\phi_{h}-\phi_{S}\right)}$ is beyond the accuracy of the low-transverse-momentum results. This is just as for $F_{U U, L}$, which is the only other structure function in (2.3) that involves purely longitudinal polarization of the virtual photon [14]. The structure function $F_{U T}^{\sin \left(3 \phi_{h}-\phi_{S}\right)}$ does not match in the low- and high- $q_{T}$ calculations. As in the case of $F_{U U}^{\cos 2 \phi_{h}}$, the low-transversemomentum result involves chiral-odd functions, whereas the high-transverse-momentum expression involves chiral-even ones. The low- $q_{T}$ result (5.89) for $F_{U T}^{\sin \left(3 \phi_{h}-\phi_{S}\right)}$ can potentially match a high-transverse-momentum calculation at twist three and order $\alpha_{s}^{2}$, and the high- $q_{T}$ result (6.11) could match with a low- $q_{T}$ calculation at twist four. Both types of calculation are beyond the current state of the art.

To the best of our knowledge, $F_{L T}^{\cos \left(\phi_{h}-\phi_{S}\right)}, F_{L T}^{\cos \phi_{S}}$ and $F_{L T}^{\cos \left(2 \phi_{h}-\phi_{S}\right)}$ have not been computed in the high- $q_{T}$ approach. From the overall factor $M$ in (5.92) to (5.94) we can 


\begin{tabular}{|l|ccc|ccc|c|}
\hline & \multicolumn{3}{|c|}{ low- $q_{T}$ calculation } & \multicolumn{3}{c|}{ high- $q_{T}$ calculation } & leading powers \\
observable & twist & order & power & twist & order & power & match \\
\hline$F_{U U, T}$ & 2 & $\alpha_{s}$ & $1 / q_{T}^{2}$ & 2 & $\alpha_{s}$ & $1 / q_{T}^{2}$ & yes \\
$F_{U U, L}$ & 4 & & & 2 & $\alpha_{s}$ & $1 / Q^{2}$ & \\
$F_{U U}^{\cos \phi_{h}}$ & 3 & $\alpha_{s}$ & $1 /\left(Q q_{T}\right)$ & 2 & $\alpha_{s}$ & $1 /\left(Q q_{T}\right)$ & yes \\
$F_{U U}^{\cos 2 \phi_{h}}$ & 2 & $\alpha_{s}$ & $1 / q_{T}^{4}$ & 2 & $\alpha_{s}$ & $1 / Q^{2}$ & no \\
$F_{L U}^{\sin \phi_{h}}$ & 3 & $\alpha_{s}^{2}$ & $1 /\left(Q q_{T}\right)$ & 2 & $\alpha_{s}^{2}$ & $1 /\left(Q q_{T}\right)$ & yes \\
$F_{U L}^{\sin \phi_{h}}$ & 3 & $\alpha_{s}^{2}$ & $1 /\left(Q q_{T}\right)$ & & & & \\
$F_{U L}^{\sin 2 \phi_{h}}$ & 2 & $\alpha_{s}$ & $1 / q_{T}^{4}$ & & & & yes \\
$F_{L L}$ & 2 & $\alpha_{s}$ & $1 / q_{T}^{2}$ & 2 & $\alpha_{s}$ & $1 / q_{T}^{2}$ & yes \\
$F_{L L}^{\cos \phi_{h}}$ & 3 & $\alpha_{s}$ & $1 /\left(Q q_{T}\right)$ & 2 & $\alpha_{s}$ & $1 /\left(Q q_{T}\right)$ & yes \\
$F_{U T, T}^{\sin \left(\phi_{h}-\phi_{S}\right)}$ & 2 & $\alpha_{s}$ & $1 / q_{T}^{3}$ & 3 & $\alpha_{s}$ & $1 / q_{T}^{3}$ & \\
$F_{U T, L}^{\sin \left(\phi_{h}-\phi_{S}\right)}$ & 4 & & & 3 & $\alpha_{s}$ & $1 /\left(Q^{2} q_{T}\right)$ & yes \\
$F_{U T}^{\sin \left(\phi_{h}+\phi_{S}\right)}$ & 2 & $\alpha_{s}$ & $1 / q_{T}^{3}$ & 3 & $\alpha_{s}$ & $1 / q_{T}^{3}$ & no \\
$F_{U T}^{\sin \left(3 \phi_{h}-\phi_{S}\right)}$ & 2 & $\alpha_{s}^{2}$ & $1 / q_{T}^{3}$ & 3 & $\alpha_{s}$ & $1 /\left(Q^{2} q_{T}\right)$ & yes \\
$F_{U T}^{\sin \phi_{S}}$ & 3 & $\alpha_{s}$ & $1 /\left(Q q_{T}^{2}\right)$ & 3 & $\alpha_{s}$ & $1 /\left(Q q_{T}^{2}\right)$ & yes \\
$F_{U T}^{\sin \left(2 \phi_{h}-\phi_{S}\right)}$ & 3 & $\alpha_{s}$ & $1 /\left(Q q_{T}^{2}\right)$ & 3 & $\alpha_{s}$ & $1 /\left(Q q_{T}^{2}\right)$ & \\
$F_{L T}^{\cos \left(\phi_{h}-\phi_{S}\right)}$ & 2 & $\alpha_{s}$ & $1 / q_{T}^{3}$ & & & & \\
$F_{L T}^{\cos \phi_{S}}$ & 3 & $\alpha_{s}$ & $1 /\left(Q q_{T}^{2}\right)$ & & & & \\
$F_{L T}^{\cos \left(2 \phi_{h}-\phi_{S}\right)}$ & 3 & $\alpha_{s}$ & $1 /\left(Q q_{T}^{2}\right)$ & & & & \\
\hline
\end{tabular}

Table 2: $\quad$ Leading power behavior of SIDIS structure functions in the intermediate region $M \ll$ $q_{T} \ll Q$, corresponding to the expansions in (1.2) and (1.4), respectively. Empty fields indicate that no calculation is available. The specification of twist 4 for $F_{U U, L}$ and $F_{U T, L}^{\sin \left(\phi_{h}-\phi_{S}\right)}$ reflects that these observables are zero when calculated at twist-two and twist-three accuracy.

only conclude that these low- $q_{T}$ results can potentially match with those of a high- $q_{T}$ calculation at twist-three accuracy.

In table 2 we collect the results for the leading power behavior of all structure functions we have discussed. We notice that for several observables the twist of the low- $q_{T}$ and the high- $q_{T}$ calculation is not the same, which is reminiscent of a similar observation we made for the high- $p_{T}$ behavior of distribution functions in section 5.3 .

\subsection{Interpolating from low to high $q_{T}$}

Let us now see how one can practically proceed when the leading terms in the low- and high$q_{T}$ descriptions of an observable do not match in the intermediate region. As an example we take the unpolarized structure function $F_{U U}^{\cos 2 \phi_{h}}$. We denote its low- $q_{T}$ approximation 
given in (5.56) by $L_{U U}^{\cos 2 \phi_{h}}$, and its high- $q_{T}$ approximation (4.26) by $H_{U U}^{\cos 2 \phi_{h}}$. Since in the intermediate region the two expressions describe distinct contributions to the cross section, one may consider to use

$$
F_{U U}^{\cos 2 \phi_{h}} \approx L_{U U}^{\cos 2 \phi_{h}}+H_{U U}^{\cos 2 \phi_{h}}
$$

as an approximation for this observable. The quality of this approximation can be assessed from the power behavior of its terms in the different regions:

$$
\begin{aligned}
L_{U U}^{\cos 2 \phi_{h}} \sim q_{T}^{2} / M^{4} & & \text { for } q_{T} \lesssim M, \\
L_{U U}^{\cos 2 \phi_{h}} \sim M^{2} / q_{T}^{4} & & \text { for } q_{T} \gg M, \\
H_{U U}^{\cos 2 \phi_{h}} \sim 1 / Q^{2} & & \text { for all } q_{T},
\end{aligned}
$$

where the behavior in (6.18) reflects that $L_{U U}^{\cos 2 \phi_{h}}$ must vanish like $q_{T}^{2}$ for $q_{T} \rightarrow 0$ due to angular momentum conservation [42]. In the intermediate region $M \ll q_{T} \ll Q$ both terms in (6.17) are required: together they give an approximation with relative corrections of order $M^{2} / q_{T}^{2}$ or $q_{T}^{2} / Q^{2}$. The relative weight of the two terms in this region is $L_{U U}^{\cos 2 \phi_{h}} / H_{U U}^{\cos 2 \phi_{h}} \sim$ $M^{2} / q_{T}^{2} \times Q^{2} / q_{T}^{2}$ and thus varies from values above to values below 1 . As an aside, let us comment on the use of a transverse-momentum-dependence like $h_{1}^{\perp}\left(x, p_{T}^{2}\right) \propto \exp \left[-c p_{T}^{2}\right]$ and $H_{1}^{\perp}\left(z, k_{T}^{2}\right) \propto \exp \left[-C k_{T}^{2}\right]$, which is often taken in phenomenological analyses. Whereas at small transverse momentum a Gaussian behavior of distribution and fragmentation functions is found to give a good description of data in many situations, it misses the perturbative tails of these functions. As a result it does not give a good approximation of $F_{U U}^{\cos 2 \phi_{h}}$ at intermediate $q_{T}$. For $M \ll q_{T} \lesssim \sqrt{M Q}$ the contribution (6.19) from the perturbative tails is actually dominant, and for $\sqrt{M Q} \lesssim q_{T} \ll Q$ it is only suppressed compared with (6.20) by a factor much larger than $M^{2} / q_{T}^{2}$.

For large $q_{T} \sim Q$ the ansatz (6.17) can be used as well: the low- $q_{T}$ calculation is not valid in this region, but the term $L_{U U}^{\cos 2 \phi_{h}}$ is power suppressed by $M^{2} / Q^{2}$ compared with the leading term $H_{U U}^{\cos 2 \phi_{h}}$, which itself provides an approximation of $F_{U U}^{\cos 2 \phi_{h}}$ up to $M^{2} / Q^{2}$ corrections. Adding $L_{U U}^{\cos 2 \phi_{h}}$ in this region hence does not spoil the accuracy of the description. Likewise, the high- $q_{T}$ calculation is not justified for $q_{T} \sim M$, but in this region the term $H_{U U}^{\cos 2 \phi_{h}}$ is suppressed by $M^{2} / Q^{2}$ compared with the correct approximation $L_{U U}^{\cos 2 \phi_{h}}$. However, one cannot use (6.17) for $q_{T} \rightarrow 0$ since $H_{U U}^{\cos 2 \phi_{h}}$ does not vanish like $q_{T}^{2}$. To repair this, one may instead take

$$
F_{U U}^{\cos 2 \phi_{h}} \approx L_{U U}^{\cos 2 \phi_{h}}+\rho\left(\frac{q_{T}^{2}}{M^{2}}\right) H_{U U}^{\cos 2 \phi_{h}}
$$

with an interpolating function $\rho(r)$ that satisfies $\rho(r) \sim r$ for $r \rightarrow 0$ and $\rho(r)-1 \sim r^{-1}$ for $r \gg 1$. A simple choice is $\rho(r)=r /(1+r)$, but obviously there are other possibilities.

An often considered observable is the azimuthal asymmetry

$$
A_{U U}^{\cos 2 \phi_{h}}=\frac{\varepsilon F_{U U}^{\cos 2 \phi_{h}}}{F_{U U, T}+\varepsilon F_{U U, L}} .
$$

Depending on $q_{T}$ we can approximate its denominator using the high- $q_{T}$ expression $H_{U U, T}+$ $\varepsilon H_{U U, L}$ from (4.23) and (4.24) or the low- $q_{T}$ result $L_{U U, T}$ given in (5.53). Since $F_{U U, L}$ is 
suppressed by $1 / Q^{2}$ for $q_{T} \ll Q$, we do not need the unknown low- $q_{T}$ expression for this structure function. Using

$$
\begin{aligned}
L_{U U, T} & \sim 1 / M^{2} & & \text { for } q_{T} \lesssim M, \\
L_{U U, T} & \sim 1 / q_{T}^{2} & & \text { for } q_{T} \gg M, \\
H_{U U, T}+\varepsilon H_{U U, L} & \sim 1 / q_{T}^{2} & & \text { for all } q_{T}
\end{aligned}
$$

together with (6.18) to (6.20), we find that

$$
A_{U U}^{\cos 2 \phi_{h}} \approx \frac{\varepsilon L_{U U}^{\cos 2 \phi_{h}}}{L_{U U, T}}+\frac{\varepsilon H_{U U}^{\cos 2 \phi_{h}}}{H_{U U, T}+\varepsilon H_{U U, L}}
$$

gives a good approximation of the asymmetry in the full $q_{T}$ range. In the intermediate region, the denominators of the two terms in (6.26) coincide up to terms of order $M^{2} / q_{T}^{2}$ or $q_{T}^{2} / Q^{2}$ and approximate $F_{U U, T}+\varepsilon F_{U U, L}$ with that precision. As discussed above, both the low- $q_{T}$ and the high- $q_{T}$ contributions are important in the intermediate region $M \ll q_{T} \ll$ $Q$ (where again one finds that with a Gaussian ansatz for the transverse-momentumdependence of distribution and fragmentation functions, the low- $q_{T}$ term would not be correctly described). For $q_{T} \sim Q$ the low- $q_{T}$ term is power suppressed and may hence be kept in (6.26). For $q_{T} \lesssim M$, the high- $q_{T}$ term in the asymmetry is suppressed by a relative factor of $M^{2} / Q^{2}$ compared with the low- $q_{T}$ term and does not degrade the quality of the approximation $(6.26)$ in the limit $q_{T} \rightarrow 0$. An additional suppression factor as in (6.21) is therefore not required. Recalling the discussion after eq. (4.33), we can understand why $\varepsilon H_{U U}^{\cos 2 \phi_{h}} /\left(H_{U U, T}+\varepsilon H_{U U, L}\right)$ has the correct $q_{T} \rightarrow 0$ limit required by angular momentum conservation: the propagator factors $1 / q_{T}^{2}$ that lead to an unphysical behavior of the individual structure functions cancel in this ratio.

Let us finally remark that the discussion in this subsection is at the level of power counting arguments. When using (6.21) or (6.26) in practice, one can explicitly check whether the terms that are out of their region of validity (the $L$ terms for $q_{T} \sim Q$ and the $H$ terms for $\left.q_{T} \lesssim M\right)$ are numerically small compared with the leading ones.

\section{Integrating over $q_{T}$}

\subsection{Behavior of integrated and weighted observables}

Up to now we have focused on the $q_{T}$-dependence of the structure functions $F\left(Q, q_{T}\right)$. As we mentioned in the introduction, observables that are integrated over $q_{T}$, with or without a weighting factor $\left(q_{T} / M\right)^{p}$, can be preferable to observables differential in $q_{T}$ for experimental reasons. Without dwelling on such practical issues, we now use our results of the previous sections for discussing the theoretical interpretation of integrated observables. As a shorthand notation we introduce

$$
\left\langle\left\langle\left(\frac{q_{T}}{M}\right)^{p} F\left(Q, q_{T}\right)\right\rangle\right\rangle=\pi z^{2} \int_{0}^{q_{\max }^{2}} d q_{T}^{2}\left(\frac{q_{T}}{M}\right)^{p} F\left(Q, q_{T}\right),
$$


where $q_{\max }$ is the upper kinematic limit of $q_{T}$, to be treated as a quantity of order $Q$ in the power counting. The prefactor has been chosen for later convenience - note that $\pi z^{2} d q_{T}^{2}$ corresponds to $d^{2} P_{h \perp}$.

To make the notion of "intermediate transverse momentum" more precise, we introduce two scales $\Gamma M^{2}$ and $\gamma Q^{2}$ such that $\Gamma \gg 1, \gamma \ll 1$, and $\Gamma M^{2}<\gamma Q^{2}$. In the intermediate region $\Gamma M^{2}<q_{T}^{2}<\gamma Q^{2}$ the results of both the low- $q_{T}$ and the high- $q_{T}$ calculations are then valid, and one can use their respective limiting expressions given in sections 5.4 and 6. It is easy to determine the power-law behavior of the contributions from the regions $q_{T}^{2}<\Gamma M^{2}$ and $q_{T}^{2}>\gamma Q^{2}$ to an integrated observable. For a single term in the general low- $q_{T}$ and high- $q_{T}$ expansions (1.1) and (1.3), we obtain

$$
\begin{aligned}
& \frac{1}{M^{2}} \int_{0}^{\Gamma M^{2}} d q_{T}^{2}\left[\frac{q_{T}}{M}\right]^{p}\left[\frac{q_{T}}{Q}\right]^{n-2} l_{n}\left(\frac{M}{q_{T}}\right) \sim\left[\frac{M}{Q}\right]^{n-2}, \\
& \frac{1}{M^{2}} \int_{\gamma Q^{2}}^{q_{\max }^{2}} d q_{T}^{2}\left[\frac{q_{T}}{M}\right]^{p}\left[\frac{M}{q_{T}}\right]^{n} h_{n}\left(\frac{q_{T}}{Q}\right) \sim\left[\frac{M}{Q}\right]^{n-2-p}
\end{aligned}
$$

from straightforward dimensional analysis. The $Q$-dependence of the integrals can thus be established without knowledge of the functions $l_{n}\left(q_{T} / M\right)$ and $h_{n}\left(q_{T} / Q\right)$ : it is directly determined by the twist $n$ in the low- $q_{T}$ case (7.2), and by the twist $n$ and the weighting power $p$ in the high- $q_{T}$ case (7.3). We observe in particular that for $p=0$, i.e. without weighting, the twist-two terms in both the low- and high- $q_{T}$ calculations give contributions to the integral that stay constant for $Q \rightarrow \infty$, whereas higher-twist terms die out in that limit. For $p>0$ the contribution from the high- $q_{T}$ region is enhanced: a twist-two term in the low- $q_{T}$ calculation will then only dominate the integral over all $q_{T}$ if for the observable in question a sufficient number of terms with low twist in the high- $q_{T}$ result are zero.

As a preparation for the discussion of azimuthal and polarization asymmetries let us first take a closer look at the familiar structure functions $F_{U U, T}$ and $F_{U U, L}$. With the behavior $F_{U U, T} \sim 1 / q_{T}^{2}$ in the intermediate region (obtained in both the low- and high- $q_{T}$ calculations), we obtain

$$
\int_{\Gamma M^{2}}^{\gamma Q^{2}} d q_{T}^{2} F_{U U, T} \sim \ln \left[\frac{\gamma}{\Gamma} \frac{Q^{2}}{M^{2}}\right]
$$

For the integral in the low- $q_{T}$ domain $q_{T}^{2}<\Gamma M^{2}$ we have the generic power-law behavior given in $(7.2)$ with $n=2$ and $p=0$. Using in addition that $F_{U U, T} \sim 1 / q_{T}^{2}$ at the upper end of the integration region, we have

$$
\int_{0}^{\Gamma M^{2}} d q_{T}^{2} F_{U U, T} \sim \ln \frac{\Gamma}{\Gamma_{0}}
$$

with some number $\Gamma_{0} \sim 1$. Likewise, we can use that $F_{U U, T} \sim 1 / q_{T}^{2}$ at the lower end of the integration region in the high- $q_{T}$ domain $q_{T}^{2}>\gamma Q^{2}$, and get

$$
\int_{\gamma Q^{2}}^{q_{\max }^{2}} d q_{T}^{2} F_{U U, T} \sim \ln \frac{\gamma_{0}}{\gamma}
$$

with some $\gamma_{0} \sim 1$. In the complete integral $\left\langle\left\langle F_{U U, T}\right\rangle\right\rangle$ all three regions in (7.4) to (7.6) thus contribute at leading power in $1 / Q$, and the dependence on the artificial separation 
parameters $\Gamma$ and $\gamma$ cancels as it should. We note that, since we are concerned with power behavior in this section, we have not taken into account logarithms of $Q / q_{T}$ in the high- or low- $q_{T}$ results for $F_{U U, T}$, which would modify the logarithms on the r.h.s. of (7.4) to (7.6).

To calculate the integrated structure function one must not double count the contributions from the low- $q_{T}$ and high- $q_{T}$ calculations in the intermediate region. Since the results of the two calculations coincide there, one may simply switch from one to the other description at a suitable point, say at $q_{T}^{2}=\gamma Q^{2}$. We can now make contact with the standard description of $q_{T}$-integrated SIDIS in the collinear factorization framework, where $\left\langle\left\langle F_{U U, T}\right\rangle\right\rangle$ is expressed in terms of the collinear functions $f_{1}(x)$ and $D_{1}(z)$. Let us in this framework take $\mu^{2}=\gamma Q^{2}$ for the factorization scale and consider the Born graph as well as the real and virtual $\alpha_{s}$-corrections, i.e., the one-loop graphs where a gluon either does or does not cross the final-state cut. Loosely speaking, the Born term then corresponds to the sum of (7.4) and (7.5), and the real corrections to (7.6). The virtual corrections correspond to the hard factor $|H|^{2}$ in the Collins-Soper factorization formula (3.3), which we neglected in section 5.3 when extracting results at lowest order in $\alpha_{s}$. The logarithm of $\gamma Q^{2}$ in (7.4) corresponds to the scale dependence of the collinear distribution and fragmentation functions, whereas the $\gamma$-dependence in (7.6) corresponds to an explicit logarithm $\ln \left(Q^{2} / \mu^{2}\right)$ in the real corrections. At the technical level, however, collinear factorization is typically implemented by using dimensional regularization instead of a transverse-momentum cutoff. The real corrections are then integrated down to $q_{T}=0$, whereas collinear distribution and fragmentation functions are defined from integrals $\int d^{2-\varepsilon} \boldsymbol{p}_{T} f_{1}\left(x, p_{T}^{2}\right)$ and $\int d^{2-\varepsilon} \boldsymbol{k}_{T} D_{1}\left(x, k_{T}^{2}\right)$ over the full transverse-momentum region. Subtractions defined for instance by the $\overline{\mathrm{MS}}$ prescription are then performed, which on one hand ensure that there is no double counting and on the other hand remove terms corresponding to logarithmic divergences in the physical limit $\varepsilon \rightarrow 0$. Since the incoming and outgoing parton momenta are approximated as collinear to the associated hadron momenta, the Born term and the virtual corrections appear with a factor $\delta^{(2)}\left(\boldsymbol{q}_{T}\right)$ in the calculation.

For the longitudinal structure function $F_{U U, L}$ the situation is quite different. Using the same procedure as for $F_{U U, T}$ we obtain

$$
\begin{aligned}
\int_{\Gamma M^{2}}^{\gamma Q^{2}} d q_{T}^{2} F_{U U, L} & \sim \gamma\left(1-\frac{\Gamma M^{2}}{\gamma Q^{2}}\right) \\
\int_{\gamma Q^{2}}^{q_{\max }^{2}} d q_{T}^{2} F_{U U, L} & \sim \gamma, \gamma_{0}-\gamma \quad \sim 1
\end{aligned}
$$

from the result (6.2) of the high- $q_{T}$ calculation. For the second step in (7.7) we have assumed that $\left(\Gamma M^{2}\right) /\left(\gamma Q^{2}\right)$ is sufficiently small compared to 1 -otherwise the corresponding integral becomes small simply because its integration region shrinks to zero. After the second step in (7.8), the dependence on $\gamma$ no longer explicitly cancels in the sum of the two integrals, but this leads to no inconsistency because (7.7) is negligible compared with (7.8). One easily sees that the parton-model approximation (6.15), whose power behavior agrees with the high- $q_{T}$ result in the intermediate region, gives a result suppressed as $M^{2} / Q^{2}$ when integrated over the low- $q_{T}$ domain $q_{T}^{2}<\Gamma M^{2}$. We thus find that the integrated structure function $\left\langle\left\langle F_{U U, L}\right\rangle\right\rangle$ is dominated by large $q_{T} \sim Q$ and can be calculated from the high- $q_{T}$ 
result alone. Moreover, one can integrate this result down to $q_{T}=0$, since the contribution from $q_{T}^{2}<\Gamma M^{2}$ is power suppressed by $M^{2} / Q^{2}$ and thus of the same order as the accuracy of the result in the high- $q_{T}$ region. Put differently, one can use the high- $q_{T}$ result extrapolated to $q_{T}^{2}<\Gamma M^{2}$ instead of the (unknown) low- $q_{T}$ result when evaluating the integrated longitudinal structure function. This is just what is done in the standard calculation using collinear factorization, where the first nonvanishing contribution to this observable starts at order $\alpha_{s}$. The integration over all $q_{T}$ of the high- $q_{T}$ expression for $F_{U U, L}$ is convergent and simply removes the $\delta$ function in the analog of (4.4). No subtraction is necessary, and correspondingly no dependence on the factorization scale $\mu$ arises at order $\alpha_{s}$.

Let us now turn to the structure functions that describe the $\phi_{h}$-dependence of the unpolarized cross section. In table 30 we see that the integrated structure function $\left\langle\left\langle F_{U U}^{\cos \phi_{h}}\right\rangle\right\rangle$ is dominated by large transverse momenta $q_{T}$, whereas the region where the low- $q_{T}$ calculation is valid contributes only as a power correction of order $M / Q$. The condition $\left(\Gamma M^{2}\right) /\left(\gamma Q^{2}\right)<1$ implies $\sqrt{\Gamma} M / Q<\sqrt{\gamma} \ll 1$, so that the factor $\sqrt{\Gamma}$ cannot compensate the suppression by $M / Q$. An important consequence is that $\left\langle\left\langle F_{U U}^{\cos \phi_{h}}\right\rangle\right\rangle$ is not a good observable to study the transverse-momentum-dependent distribution and fragmentation functions appearing in the low- $q_{T}$ result (5.55). An appropriate observable for this purpose is the structure function differential in $q_{T}$. If integration over $q_{T}$ is required by statistics, one should impose a suitable upper cutoff on the integral. According to table 3 , the dependence of the integral on this cutoff is not negligible and must hence explicitly be kept in the theoretical calculation. Note that in order not to introduce an artificial $\phi_{h}$-dependence, the cutoff should be imposed on $q_{T}^{2}$, or equivalently on $P_{h \perp}^{2}$, but not on a transverse momentum w.r.t. the lepton beam axis.

Integrated observables which are weighted with a suitable power of $q_{T} / M$ have the desirable property that the transverse-momentum convolutions (5.52) in the low- $q_{T}$ results factorize into separate integrals over either distribution or fragmentation functions [65, 12]. With $\hat{\boldsymbol{h}}=-\boldsymbol{q}_{T} / q_{T}$ one readily finds from (5.55) that $\int d q_{T}^{2}\left(q_{T} / M\right) F_{U U}^{\cos \phi_{h}}$ formally factor-

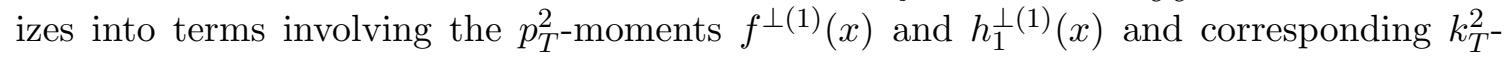
moments of fragmentation functions. However, this deconvolution only takes place if one integrates over all $q_{T}$ up to infinity. This is clearly inadequate because $\left(q_{T} / M\right) F_{U U}^{\cos \phi_{h}}$ becomes constant for $q_{T} \gg M$. A reflection of this is the fact that the $p_{T}^{2}$-moment $f^{\perp(1)}(x)$ involves a quadratic divergence at large $p_{T}$, as we already noted in section 5.3. Moreover, we see in table 3 that the contribution from the low- $q_{T}$ region to $\left\langle\left\langle\left(q_{T} / M\right) F_{U U}^{\cos \phi_{h}}\right\rangle\right\rangle$ is power suppressed by $M^{2} / Q^{2}$ compared with the contribution from $q_{T} \sim Q$, so that this observable is even less well suited to study small $q_{T}$ than the unweighted structure function. Conversely, the weighted structure function is a good observable for studying large $q_{T}$. The high- $q_{T}$ expression for $F_{U U}^{\cos \phi_{h}}$ depends on the same collinear functions $f_{1}(x)$ and $D_{1}(z)$ as $F_{U U, T}$ but involves different hard-scattering kernels, so that $F_{U U}^{\cos \phi_{h}}$ provides an additional observable if one aims, for instance, at separating the fragmentation functions for different quark and antiquark flavors and the gluon, or at testing the adequacy of the theoretical description. Up to corrections of order $M^{2} / Q^{2}$ one can evaluate $\left\langle\left\langle\left(q_{T} / M\right) F_{U U}^{\cos \phi_{h}}\right\rangle\right\rangle$ from the high- $q_{T}$ result alone, which in addition may be integrated down to $q_{T}=0$. One then obtains a simple expression, just as in the analogous case of $\left\langle\left\langle F_{U U, L}\right\rangle\right\rangle$. The unweighted 


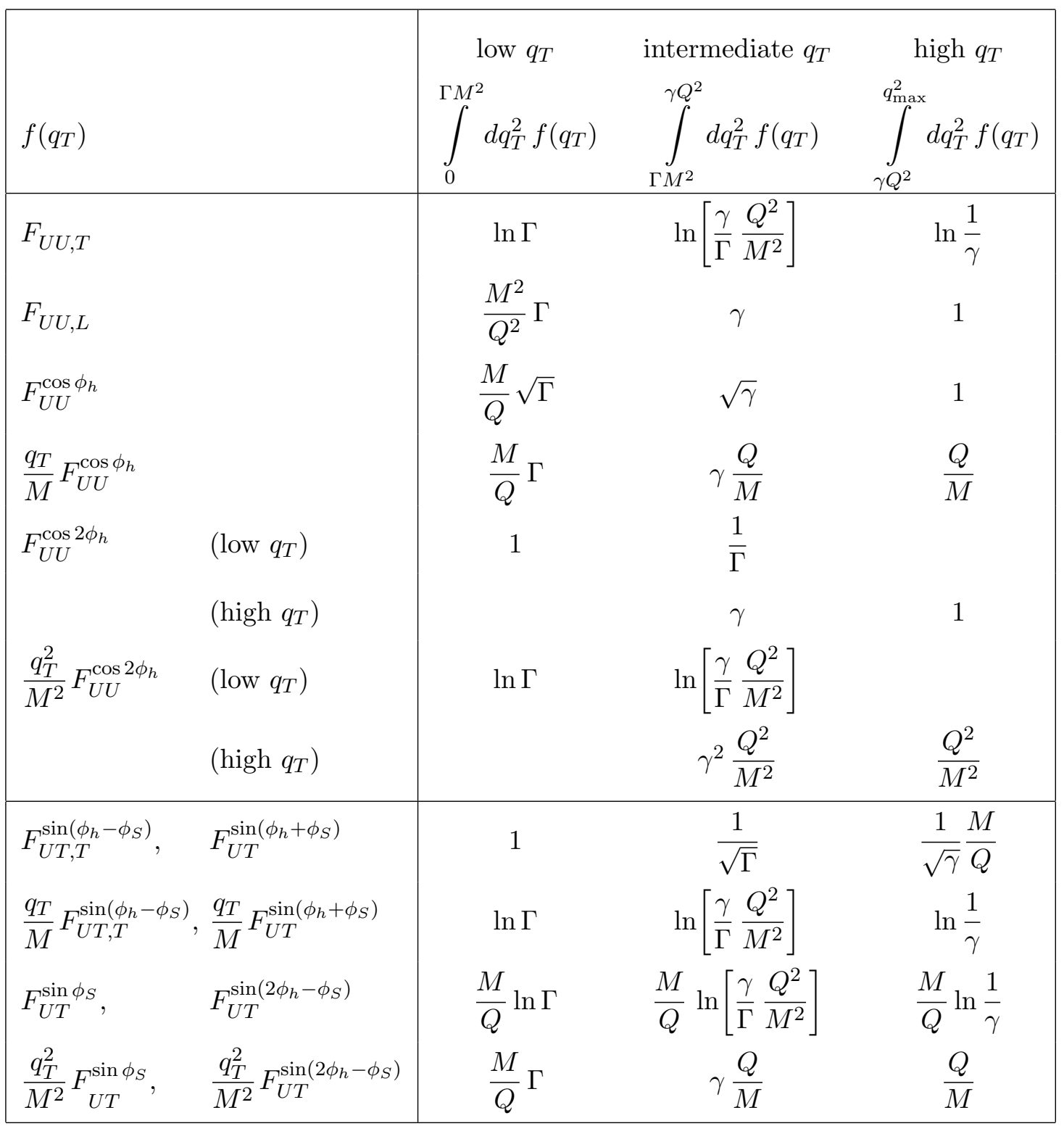

Table 3: Behavior of selected observables integrated over different regions of $q_{T}^{2}$. It is assumed that $\Gamma \gg 1, \gamma \ll 1$ and that $\left(\Gamma M^{2}\right) /\left(\gamma Q^{2}\right)$ is sufficiently small compared to 1 . In cases where the low- $q_{T}$ and high- $q_{T}$ calculations do not match in the intermediate region, their respective contributions are given in separate rows. The low- $q_{T}$ entry for $F_{U U, L}$ corresponds to the parton-model approximation in 6.15$)$.

integral $\left\langle\left\langle F_{U U}^{\cos \phi_{h}}\right\rangle\right\rangle$ is less attractive for studying the high- $q_{T}$ result since the contribution from the low- $q_{T}$ region is only suppressed by $M / Q$. To evaluate that contribution is difficult in practice as it contains transverse-momentum-dependent distribution and fragmentation functions that are poorly known. If the weighted integral and the differential structure function are affected with large experimental uncertainties, one may instead have to consider the integral of $F_{U U}^{\cos \phi_{h}}$ with a lower cutoff on $q_{T}$. This was for instance done in [17] and [62, 66]. 
As discussed in the previous section, the structure function $F_{U U}^{\cos 2 \phi_{h}}$ receives contributions from the low- $q_{T}$ and high- $q_{T}$ calculations which do not match in the intermediate region and have distinct dynamical origins, given that they respectively involve chiral-odd and chiral-even distribution and fragmentation functions. As we see in table 3, both mechanisms contribute to the integrated structure function at leading power, with only moderate contributions from intermediate $q_{T}$. For calculating the integrated structure function it is appropriate to add the contributions from the two mechanisms. Furthermore, it is consistent to perform the $q_{T}$-integral over the entire kinematical region for both mechanisms, i.e. without introducing cutoff parameters, given that contributions from regions where the approaches are not valid (low $q_{T}$ for the high- $q_{T}$ calculation and vice versa) are powersuppressed by $M^{2} / Q^{2}$. This is similar to the case of the interpolation formula 6.17) discussed in the previous subsection, but for the integrated structure function the unphysical behavior of the high- $q_{T}$ result in the limit $q_{T} \rightarrow 0$ does not matter, at least at the level of power counting. The weighted structure function $\left\langle\left\langle\left(q_{T} / M\right)^{2} F_{U U}^{\cos 2 \phi_{h}}\right\rangle\right\rangle$ has been proposed for obtaining a low- $q_{T}$ result in terms of the moments $h_{1}^{\perp(1)}(x)$ and $H_{1}^{\perp(1)}(z)$ of the Boer-Mulders and the Collins functions, without any convolution of transverse-momentumdependent factors [12]. According to table 3 this observable is, however, dominated by $q_{T} \sim Q$ and only sensitive to $h_{1}^{\perp(1)}(x)$ and $H_{1}^{\perp(1)}(z)$ at the level of $M^{2} / Q^{2}$ corrections. Such contributions are not under control in the integrated observable, because uncalculated corrections of the same size appear in the high- $q_{T}$ region as well. Neglecting $M^{2} / Q^{2}$ corrections, one can evaluate $\left\langle\left\langle\left(q_{T} / M\right)^{2} F_{U U}^{\cos 2 \phi_{h}}\right\rangle\right\rangle$ as an integral of the high- $q_{T}$ expression over the full $q_{T}$ domain. In the same way as $\left\langle\left\langle\left(q_{T} / M\right) F_{U U}^{\cos \phi_{h}}\right\rangle\right\rangle$, this provides an independent observable sensitive to the twist-two functions $f_{1}(x)$ and $D_{1}(z)$.

\subsection{Polarization dependence}

Among the many observables for polarized SIDIS, the structure functions $F_{U T, T}^{\sin \left(\phi_{h}-\phi_{S}\right)}$ and $F_{U T}^{\sin \left(\phi_{h}+\phi_{S}\right)}$ have received particular attention in the recent literature. According to the low- $q_{T}$ results (5.62) and (5.64), they provide access to the Sivers function $f_{1 T}^{\perp}$ in the first and to the transversity distribution $h_{1}$ and the Collins function $H_{1}^{\perp}$ in the second case [67]. Both structure functions have been found to be of significant size in HERMES measurements on a proton target [20].

As we see in table 3 , the integrated structure function $\left\langle\left\langle F_{U T, T}^{\sin \left(\phi_{h}-\phi_{S}\right)}\right\rangle\right\rangle$ is dominated by the low- $q_{T}$ region and can hence be used for extracting information about $f_{1 T}^{\perp}\left(x, p_{T}^{2}\right)$. The high- $q_{T}$ region is however only suppressed by $M / Q$, so that it may be of advantage to impose an upper cutoff on the $q_{T}$ integral in such analysis. The weighted structure function $\left\langle\left\langle\left(q_{T} / M\right) F_{U T, T}^{\sin \left(\phi_{h}-\phi_{S}\right)}\right\rangle\right\rangle$ receives contributions from both high and low $q_{T}$ at leading order in $M / Q$. One can thus compute the weighted integral by switching from one to the other formulation at some $q_{T}$. To achieve a factorization of the transverse-momentum convolution in the low- $q_{T}$ expression, one should however integrate it over all $q_{T}$ up to infinity. Since $\left(q_{T} / M\right) F_{U T, T}^{\sin \left(\phi_{h}-\phi_{S}\right)}$ behaves as $1 / q_{T}^{2}$ for $q_{T} \gg M$, a suitable regularization is required. This suggests a procedure akin to the description of $\left\langle\left\langle F_{U U, T}\right\rangle\right\rangle$ in collinear factorization, which we reviewed in the previous subsection. As dimensional regularization preserves rotation invariance in the transverse plane, the integral over all $q_{T}$ of the weighted low- $q_{T}$ 
result (5.62) turns into the product

$$
\left\langle\left\langle\left(q_{T} / M\right) F_{U T, T}^{\sin \left(\phi_{h}-\phi_{S}\right)}\right\rangle=-2 \sum_{a} x e_{a}^{2} f_{1 T}^{a \perp(1)}(x ; Q) D_{1}^{a}(z ; Q)\right.
$$

of collinear functions defined in the $\overline{\mathrm{MS}}$ scheme. One can trade $f_{1 T}^{\perp(1)}(x)$ for the twist-three function $G_{F}(x, x)$, which appears in the high- $q_{T}$ calculation [44, 0, 8]. In order to integrate the high- $q_{T}$ result down to $q_{T}=0$, one must extend it to $4-\varepsilon$ dimensions and perform the necessary $\overline{\mathrm{MS}}$ subtractions. Adding graphs with virtual corrections to the hard-scattering subprocess (which give the hard factor $|H|^{2}$ in the Collins-Soper formalism) one will obtain a complete NLO result in $\alpha_{s}$. Such a procedure would be the analog of a standard NLO computation for integrated observables within collinear factorization at twist-two level. Note that (7.9) gives a consistent approximation of the weighted structure function at LO in $\alpha_{s}$, in analogy to the well-known tree-level expression $\left\langle\left\langle F_{U U, T}\right\rangle\right\rangle=\sum_{a} x e_{a}^{2} f_{1}^{a}(x) D_{1}^{a}(z)$. The factorization scale $\mu$ of the functions in (7.9) has been set to $Q$ in order to avoid large logarithms of $Q / \mu$ appearing in the $\alpha_{s}$-corrections. To leading order, the logarithmic $Q$ dependence of the weighted structure function then follows from the evolution equations for $D_{1}(z)$ and $f_{1 T}^{\perp(1)}(x)$. The latter have been investigated in [57].

The situation for the structure function $F_{U T}^{\sin \left(\phi_{h}+\phi_{S}\right)}$ is the same as for $F_{U T, T}^{\sin \left(\phi_{h}-\phi_{S}\right)}$ since the power behavior of these observables coincides in both the low- and high- $q_{T}$ calculations. The evaluation of the weighted structure function in collinear factorization gives

$$
\left\langle\left\langle\left(q_{T} / M_{h}\right) F_{U T}^{\sin \left(\phi_{h}+\phi_{S}\right)}\right\rangle=2 \sum_{a} x e_{a}^{2} h_{1}^{a}(x ; Q) H_{1}^{a \perp(1)}(z ; Q)\right.
$$

for the Born term. The $k_{T}^{2}$-moment $H_{1}^{\perp(1)}(z)$ is related to the twist-three fragmentation function $\widehat{E}_{F}$ appearing at order $\alpha_{s}$. We note that according to the high- $q_{T}$ results in [43, 44] the $\alpha_{s}$-corrections to both $(7.9)$ and $(7.10)$ involve each of the twist-three functions $G_{F}$, $\widetilde{G}_{F}$, and $\widehat{E}_{F}$.

According to table 3, the integrated structure functions $\left\langle\left\langle F_{U T}^{\sin \phi_{S}}\right\rangle\right\rangle$ and $\left\langle\left\langle F_{U T}^{\sin \left(2 \phi_{h}-\phi_{S}\right)}\right\rangle\right\rangle$ receive comparable contributions from all regions of $q_{T}$. Weighting the structure functions with $\left(q_{T} / M\right)^{2}$ one obtains integrals that can be evaluated in the high- $q_{T}$ formalism up to corrections of order $M^{2} / Q^{2}$, similarly to the case of $\left\langle\left\langle\left(q_{T} / M\right) F_{U U}^{\cos \phi_{h}}\right\rangle\right\rangle$ we discussed in the previous subsection. The high- $q_{T}$ expressions computed in [43, 44] imply that $\left\langle\left\langle\left(q_{T} / M\right)^{2} F_{U T}^{\sin \left(2 \phi_{h}-\phi_{S}\right)}\right\rangle\right\rangle$ is sensitive to $G_{F}$ and $\widetilde{G}_{F}$, whereas $\left\langle\left\langle\left(q_{T} / M\right)^{2} F_{U T}^{\sin \phi_{S}}\right\rangle\right\rangle$ also depends on $\widehat{E}_{F}$. Whether these observables are large enough to be measured in practice is, of course, a different question.

From (5.89) we can infer that the integral of $F_{U T}^{\sin \left(3 \phi_{h}-\phi_{S}\right)}$ receives a contribution of order 1 from low $q_{T}$, whereas the high- $q_{T}$ result (6.11) is suppressed by $M / Q$. According to (5.65) the integrated structure function $\left\langle\left\langle F_{U T}^{\sin \left(3 \phi_{h}-\phi_{S}\right)}\right\rangle\right.$ may hence be used to extract information on $h_{1 T}^{\perp}\left(x, p_{T}\right)$ and on the Collins fragmentation function, with the same caveat we discussed for $\left\langle\left\langle F_{U T, T}^{\sin \left(\phi_{h}-\phi_{S}\right)}\right\rangle\right.$. To obtain an integral that is dominated by the high- $q_{T}$ result up to $M^{2} / Q^{2}$ corrections, one must weight $F_{U T}^{\sin \left(3 \phi_{h}-\phi_{S}\right)}$ with $\left(q_{T} / M\right)^{3}$.

Let us now turn to observables that involve longitudinal polarization. Similarly to $F_{U U}^{\cos \phi_{h}}$, the lepton-helicity-dependent structure function $F_{L U}^{\sin \phi_{h}}$ for an unpolarized target 


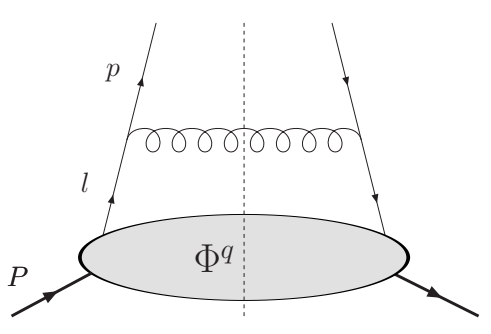

(a)

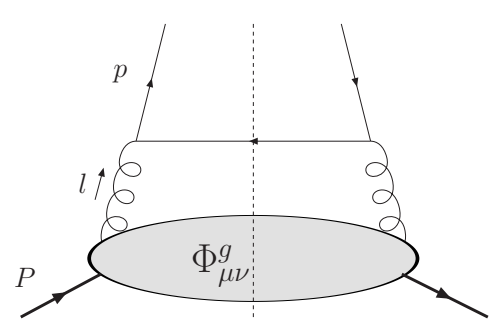

(b)

Figure 5: Diagrams for the calculation of the leading high- $p_{T}$ behavior of the quark-quark correlator $\Phi\left(x, p_{T}\right)$ in axial gauge $A \cdot v=0$.

receives a contribution of order $M / Q$ from low $q_{T}$ and of order unity from high $q_{T}$. It is therefore in principle suitable for investigating the high- $q_{T}$ result of Hagiwara et al. [45]. However, the contribution from large $q_{T}$ comes with a factor $\alpha_{s}^{2}$ in this case, which may not be sufficient for neglecting power-suppressed contributions from low $q_{T}$ in practice. From this point of view, it would be advantageous to weight the structure function with $q_{T} / M$, or to integrate over $q_{T}$ starting from a lower cutoff.

Finally, the structure functions $F_{L L}$ and $F_{L L}^{\cos \phi_{h}}$ have the same power behavior as their unpolarized counterparts $F_{U U, T}$ and $F_{U U}^{\cos \phi_{h}}$, and their discussion is analogous to the one in the previous subsection. In particular, the weighted integral $\left\langle\left\langle\left(q_{T} / M\right) F_{L L}^{\cos \phi_{h}}\right\rangle\right\rangle$ depends on the polarized parton densities $g_{1}$ and, if measurable with sufficient accuracy, could be used in addition to the well-known observable $\left\langle\left\langle F_{L L}\right\rangle\right\rangle$ for disentangling the contributions from different quark and antiquark flavors and from the gluon.

\section{From low to intermediate $q_{T}$ : explicit calculation}

In this section we compute the high-transverse-momentum tails of the quark distributions in (5.44) and of the analogous fragmentation functions. These are the functions which appear at lowest order in the $1 / p_{T}$ expansion of section 5.3 and are hence expressed in terms of collinear functions of twist two. While in section 6 we identified observables whose power behavior agrees in the low- and high- $q_{T}$ calculations, we will then be able to check for selected structure functions whether agreement is also found for their explicit expressions.

\subsection{High- $p_{T}$ tails of distribution functions}

Let us begin with the quark distribution functions. We work in the original scheme of Collins and Soper [24], using a spacelike axial gauge with the singularities of the gluon propagator regulated by the principal value prescription. The only Feynman diagrams to be evaluated are then those depicted in figure $5 \mathrm{a}$ and $\mathrm{b}$. For further discussion and a comparison with the calculation in Feynman gauge, we refer to appendix A. 
The contribution of the quark-to-quark term shown in figure fa reads

$$
\begin{aligned}
\left.\Phi^{q}\left(x, p_{T}\right)\right|_{\left(\Phi^{2}\right)}= & \frac{4 \pi \alpha_{s}}{(2 \pi)^{3}} C_{F} \int d p^{-} \int d l^{+} \delta\left((l-p)^{2}\right) \theta\left(l^{+}-p^{+}\right) \\
& \times\left. d^{\mu \nu}(l-p ; v) \frac{\not p}{p^{2}} \gamma_{\nu} \Phi_{2}^{q}\left(\frac{x}{\hat{x}}\right) \gamma_{\mu} \frac{\not p}{p^{2}}\right|_{l^{-}=0, \boldsymbol{l}_{T}=\mathbf{0}_{T}},
\end{aligned}
$$

where it is understood that $p^{+}=x P^{+}$and $l^{+}=p^{+} / \hat{x}$. As explained in section 5.3, the restriction to leading order in $1 / p_{T}$ allows us to set $l^{-}$and $\boldsymbol{l}_{T}$ to zero when calculating the hard-scattering subprocess, and to retain only the twist-two part $\Phi_{2}^{q}(x / \hat{x})$ of the collinear quark-quark correlator at the bottom of the graph. The gluon polarization sum in $A \cdot v=0$ gauge is given by

$$
d^{\mu \nu}(q ; v)=-g^{\mu \nu}+\frac{q^{\mu} v^{\nu}+q^{\nu} v^{\mu}}{q \cdot v}-\frac{q^{\mu} q^{\nu}}{(q \cdot v)^{2}} v^{2}
$$

where the singularities at $q \cdot v=0$ are to be regulated by the principal value prescription. Using the $\delta$-function to perform the $p^{-}$integration,

$$
\left.\delta\left((l-p)^{2}\right)\right|_{l^{-}=0, \boldsymbol{l}_{T}=\mathbf{0}_{T}}=\frac{\hat{x}}{2 p^{+}(1-\hat{x})} \delta\left(p^{-}+\frac{\boldsymbol{p}_{T}^{2}}{2 p^{+}} \frac{\hat{x}}{1-\hat{x}}\right)
$$

we obtain

$$
\left.\Phi^{q}\left(x, p_{T}\right)\right|_{\text {(国) }}=\frac{\alpha_{s}}{(2 \pi)^{2}} C_{F} \frac{1}{p_{T}^{4}} \int_{x}^{1} \frac{d \hat{x}}{\hat{x}}(1-\hat{x}) d^{\mu \nu}(\bar{l}-\bar{p} ; v) \not \supset \gamma_{\nu} \Phi_{2}^{q}\left(\frac{x}{\hat{x}}\right) \gamma_{\mu} \not \vec{p},
$$

where we have introduced the notation

$$
\begin{aligned}
& \bar{p}=p^{+} n_{+}-\frac{p_{T}^{2}}{2 p^{+}} \frac{\hat{x}}{1-\hat{x}} n_{-}+p_{T}, \\
& \bar{l}=\frac{p^{+}}{\hat{x}} n_{+}
\end{aligned}
$$

for the approximated momenta in the hard-scattering kernel. We note that the virtuality of the upper quark legs

$$
\bar{p}^{2}=-\frac{\boldsymbol{p}_{T}^{2}}{1-\hat{x}}
$$

is always spacelike. The gauge fixing vector can be written as

$$
v=v^{-} n_{-}-\frac{2\left(P^{+}\right)^{2} v^{-}}{\zeta} n_{+}
$$

with

$$
\zeta=-\frac{(2 P \cdot v)^{2}}{v^{2}}=-\frac{2\left(P^{+}\right)^{2} v^{-}}{v^{+}}
$$

where in the second step we have neglected $M^{2}$ compared with $\zeta$. We therefore have

$$
(\bar{l}-\bar{p}) \cdot v=(\bar{l}-\bar{p})^{+} v^{-}-\frac{2\left(p^{+}\right)^{2}}{x^{2} \zeta}(\bar{l}-\bar{p})^{-} v^{-}=\frac{1}{\hat{x}}\left[1-\hat{x}-\eta \frac{\hat{x}^{2}}{1-\hat{x}}\right] p^{+} v^{-},
$$


where we have introduced the parameter

$$
\eta=\frac{\boldsymbol{p}_{T}^{2}}{x^{2} \zeta}=-\frac{\boldsymbol{p}_{T}^{2}}{2\left(p^{+}\right)^{2}} \frac{v^{+}}{v^{-}} .
$$

We now decompose the gluon polarization sum as

$$
d^{\mu \nu}(\bar{l}-\bar{p} ; v)=\sum_{i=1}^{4} d_{(i)}^{\mu \nu}(\bar{l}-\bar{p} ; v),
$$

with

$$
\begin{aligned}
& d_{(1)}^{\mu \nu}(\bar{l}-\bar{p} ; v)=\frac{1-\hat{x}}{1-\hat{x}-\eta \frac{\hat{x}^{2}}{1-\hat{x}}} d^{\mu \nu}\left(\bar{l}-\bar{p} ; n_{-}\right), \\
& d_{(2)}^{\mu \nu}(\bar{l}-\bar{p} ; v)=\eta \frac{\hat{x}^{2}}{1-\hat{x}} \frac{g^{\mu \nu}}{1-\hat{x}-\eta \frac{\hat{x}^{2}}{1-\hat{x}}}, \\
& d_{(3)}^{\mu \nu}(\bar{l}-\bar{p} ; v)=-\eta \frac{2 \hat{x} p^{+}}{\boldsymbol{p}_{T}^{2}} \frac{(\bar{l}-\bar{p})^{\mu} n_{+}^{\nu}+(\bar{l}-\bar{p})^{\nu} n_{+}^{\mu}}{1-\hat{x}-\eta \frac{\hat{x}^{2}}{1-\hat{x}}} \\
& d_{(4)}^{\mu \nu}(\bar{l}-\bar{p} ; v)=\eta \frac{4 \hat{x}^{2}}{\boldsymbol{p}_{T}^{2}} \frac{(\bar{l}-\bar{p})^{\mu}(\bar{l}-\bar{p})^{\nu}}{\left[1-\hat{x}-\eta \frac{\hat{x}^{2}}{1-\hat{x}}\right]^{2}} .
\end{aligned}
$$

Notice that the first term 8.13$)$ is proportional to the polarization sum $d^{\mu \nu}\left(\bar{l}-\bar{p} ; n_{-}\right)$ one would use when calculating in light-cone gauge $A \cdot n_{-}=0$. We will see shortly that the prefactor in (8.13) regulates the divergence at $\hat{x}=1$ which would arise in that gauge. From the parameterization (5.13) we readily see that the twist-two part of the quark-quark correlator satisfies $\not n_{+} \Phi_{2}^{q}=\Phi_{2}^{q} \not h_{+}=0$, so that terms with $n_{+}^{\mu}$ or $n_{+}^{\nu}$ in $d^{\mu \nu}$ vanish when inserted into (8.4). With $\bar{l}$ being proportional to $n_{+}$, we hence need only the first two terms and the $\bar{p}^{\mu} \bar{p}^{\nu}$ part of the last term in the decomposition (8.12). This gives

$$
\begin{aligned}
\left.\Phi^{q}\left(x, p_{T}\right)\right|_{\text {国 }}= & \frac{\alpha_{s}}{(2 \pi)^{2}} C_{F} \frac{1}{p_{T}^{2}} \int_{x}^{1} \frac{d \hat{x}}{\hat{x}} \\
& \times\left[\frac{1-\hat{x}}{(1-\hat{x})^{2}-\eta \hat{x}^{2}} \frac{1}{p_{T}^{2}}(1-\hat{x})^{2} d^{\mu \nu}\left(\bar{l}-\bar{p} ; n_{-}\right) \not \supset p \gamma_{\nu} \Phi_{2}^{q}\left(\frac{x}{\hat{x}}\right) \gamma_{\mu} \not \vec{p}\right. \\
& +\frac{\eta}{(1-\hat{x})^{2}-\eta \hat{x}^{2}} \frac{\hat{x}^{2}}{\boldsymbol{p}_{T}^{2}}(1-\hat{x}) \not p \gamma^{\mu} \Phi_{2}^{q}\left(\frac{x}{\hat{x}}\right) \gamma_{\mu} \not \supset p \\
& \left.+\frac{\eta(1-\hat{x})}{\left[(1-\hat{x})^{2}-\eta \hat{x}^{2}\right]^{2}} 4 \hat{x}^{2} \Phi_{2}^{q}\left(\frac{x}{\hat{x}}\right)\right] .
\end{aligned}
$$

To proceed we must determine the behavior of the different terms in the limit $\hat{x} \rightarrow 1$, where $\bar{p}^{-} \sim(1-\hat{x})^{-1}$ becomes singular. Using the form (8.2) with $v$ replaced by $n_{-}$, we obtain

$$
\begin{aligned}
& (1-\hat{x})^{2} d^{\mu \nu}\left(\bar{l}-\bar{p} ; n_{-}\right) \not \supset p \gamma_{\nu} \Phi_{2}^{q} \gamma_{\mu} \not \supset p \\
& =-(1-\hat{x})^{2} \not{p} \gamma^{\mu} \Phi_{2}^{q} \gamma_{\mu} \not p+\frac{\hat{x} \boldsymbol{p}_{T}^{2}}{p^{+}}\left(\not p h h_{-} \Phi_{2}^{q}+\Phi_{2}^{q} \not h_{-} \not \vec{p}\right) .
\end{aligned}
$$


Since the minus-component of $\bar{p}$ drops out in $\not p \not h_{-}$and $\not h_{-} \not p$, the expression in (8.18) is finite for $\hat{x} \rightarrow 1$. For the second term in (8.17) we have

$$
(1-\hat{x}) \not \supset \gamma^{\mu} \Phi_{2}^{q} \gamma_{\mu} \not \supset p=-(1-\hat{x}) \not \supset\left(f_{1}^{q} \not h_{+}-g_{1}^{q} S_{L} \gamma_{5} \not h_{+}\right) \not \supset
$$

after plugging in the parameterization of $\Phi_{2}^{q}$ from (5.13). This contains a piece with two factors of $\bar{p}^{-}$, which is proportional to the Dirac matrices $\not_{-}$or $\gamma_{5} \not_{-}$. According to the decomposition (5.4) it therefore does not contribute to the twist-two or twist-three parts of the correlator $\Phi\left(x, p_{T}\right)$, on which we concentrate here. In the twist-four part of (8.1) this piece leads to a singularity at $\hat{x}=1$, or in other words at $\bar{p}^{-} \rightarrow-\infty$, showing that at twist-four level the $A \cdot v=0$ gauge is insufficient to render the integral over $p^{-}$in $\Phi\left(x, p_{T}\right)=\int d p^{-} \Phi(p)$ well defined.

In the following we take the limit $\boldsymbol{p}_{T}^{2} \ll \zeta$, corresponding to $\eta \ll 1$. The motivation for this is that in physical processes we need the correlator $\Phi\left(x, p_{T} ; \zeta\right)$ for $\boldsymbol{p}_{T}^{2} \ll Q^{2}$ and $x^{2} \zeta \sim Q^{2}$, as discussed in section 3.1. We note that in a frame where $x P^{+} \sim Q$ this corresponds to $v^{+} \sim v^{-}$. According to (8.11) the parameter $\sqrt{\eta}$ is then proportional to the small angle between the quark momentum $p$ and the hadron momentum $P$, with a factor of proportionality of order 1 . Notice that at this point we introduce a hierarchy in size between $p_{T}$ and $P^{+}$, which were not distinguished in the power counting of section 5.3 . This is similar to what we have done with the high- $q_{T}$ calculation of structure functions in section $\theta^{4}$ : we started with the result (4.4), which is derived without making a distinction between the size of $q_{T}$ and $Q$, and in a second step we took its limit for $q_{T} \ll Q$.

For $\eta \ll 1$ the first term in the square brackets of (8.17) can be rewritten by using that for any function $G(\hat{x})$ which is regular at $\hat{x}=1$

$$
\lim _{\eta \rightarrow 0} \mathrm{PV} \int_{x}^{1} d \hat{x} \frac{1-\hat{x}}{(1-\hat{x})^{2}-\eta \hat{x}^{2}} G(\hat{x})=\int_{x}^{1} d \hat{x} \frac{G(\hat{x})}{(1-\hat{x})_{+}}+\frac{1}{2} G(1) \ln \frac{1}{|\eta|},
$$

where the plus-distribution is defined as in (4.22). From

$$
\lim _{\eta \rightarrow 0} \mathrm{PV} \int_{x}^{1} d \hat{x} \frac{\eta}{(1-\hat{x})^{2}-\eta \hat{x}^{2}} G(\hat{x})=0
$$

we see that the second term in (8.17) does not contribute in the small- $\eta$ limit when restricted to the twist-two and twist-three parts of $\Phi\left(x, p_{T}\right)$. In contrast, the third term in 8.17) does contribute, since

$$
\lim _{\eta \rightarrow 0} \mathrm{PV} \int_{x}^{1} d \hat{x} \frac{\eta(1-\hat{x})}{\left[(1-\hat{x})^{2}-\eta \hat{x}^{2}\right]^{2}} G(\hat{x})=-\frac{1}{2} G(1)
$$

Therefore, our final result reads

$$
\begin{aligned}
\left.\Phi^{q}\left(x, p_{T}\right)\right|_{\text {国 } \mathrm{a})}= & \frac{\alpha_{s}}{2 \pi^{2}} C_{F} \frac{1}{\boldsymbol{p}_{T}^{2}} \int_{x}^{1} \frac{d \hat{x}}{\hat{x}}\left\{\left[\frac{1}{(1-\hat{x})_{+}}+\frac{1}{2} \delta(1-\hat{x}) \ln \frac{1}{\eta}\right]\right. \\
& \left.\times \frac{(1-\hat{x})^{2}}{2 \boldsymbol{p}_{T}^{2}} d^{\mu \nu}\left(\bar{l}-\bar{p} ; n_{-}\right) \not p \gamma_{\nu} \Phi_{2}^{q}\left(\frac{x}{\hat{x}}\right) \gamma_{\mu} \not p-\delta(1-\hat{x}) \Phi_{2}^{q}\left(\frac{x}{\hat{x}}\right)\right\}
\end{aligned}
$$


to leading order in $1 / p_{T}$, where it is understood that we have restricted ourselves to the twist-two and twist-three parts of the correlator on the l.h.s. We note at this point that if we work with a timelike axial gauge, i.e. with negative $\zeta$ and $\eta$ in (8.8) to (8.11), we obtain the same result as in 8.23 with $\ln \left(-\eta^{-1}\right)$ instead of $\ln \left(\eta^{-1}\right)$. The polarization sum $d^{\mu \nu}(\bar{l}-\bar{p} ; v)$ is then nonsingular in the whole region $x \leq \hat{x} \leq 1$, and the principal value prescription in $(8.20)$ to $(8.22)$ is not required. A timelike vector $v$ was indeed used for the construction of factorization by Ji et al. [26], whereas arguments in favor of taking $v$ spacelike were given by Collins and Metz in [50].

The gluon-to-quark contribution to the correlation function comes from the diagram in figure 5b. Its calculation is simpler than the previous one, due to the absence of a gluon polarization sum in axial gauge. Correspondingly, the result is independent of $\eta$. The counterpart of the expression in (8.4) now reads

$$
\left.\Phi^{q}\left(x, p_{T}\right)\right|_{(5 \mathrm{~b})}=\frac{\alpha_{s}}{(2 \pi)^{2}} T_{R} \frac{1}{\boldsymbol{p}_{T}^{4}} \int_{x}^{1} \frac{d \hat{x}}{\hat{x}}(1-\hat{x}) \Phi_{2}^{g, \mu \nu}\left(\frac{x}{\hat{x}}\right) \not p \gamma_{\nu}(\bar{\gamma}-\not ึ) \gamma_{\mu} \not \supset,
$$

where the twist-two part of the collinear gluon correlation function is given by

$$
\Phi_{2}^{g, \mu \nu}(x)=\frac{1}{2 x P^{+}}\left\{-g_{T}^{\mu \nu} f_{1}^{g}(x)+i \epsilon_{T}^{\mu \nu} S_{L} g_{1}^{g}(x)\right\},
$$

see e.g. [68]. Inserting (8.5) and (8.6) and using some Dirac algebra, one finds that the integrand of (8.24) is finite at $\hat{x}=1$.

From (8.23) and (8.24) we can easily project out the contributions to the individual terms in the decomposition (5.4) of $\Phi^{q}\left(x, p_{T}\right)$. For the high- $p_{T}$ behavior of the unpolarized distributions we obtain

$$
\begin{aligned}
f_{1}^{q}\left(x, p_{T}^{2}\right) & =\frac{\alpha_{s}}{2 \pi^{2}} \frac{1}{\boldsymbol{p}_{T}^{2}}\left[\frac{L\left(\eta^{-1}\right)}{2} f_{1}^{q}(x)-C_{F} f_{1}^{q}(x)+\left(P_{q q} \otimes f_{1}^{q}+P_{q g} \otimes f_{1}^{g}\right)(x)\right], \\
x f^{\perp q}\left(x, p_{T}^{2}\right) & =\frac{\alpha_{s}}{2 \pi^{2}} \frac{1}{2 p_{T}^{2}}\left[\frac{L\left(\eta^{-1}\right)}{2} f_{1}^{q}(x)+\left(P_{q q}^{\prime} \otimes f_{1}^{q}+P_{q g}^{\prime} \otimes f_{1}^{g}\right)(x)\right],
\end{aligned}
$$

whereas for the polarized distributions we find

$$
\begin{aligned}
g_{1 L}^{q}\left(x, p_{T}^{2}\right) & =\frac{\alpha_{s}}{2 \pi^{2}} \frac{1}{\boldsymbol{p}_{T}^{2}}\left[\frac{L\left(\eta^{-1}\right)}{2} g_{1}^{q}(x)-C_{F} g_{1}^{q}(x)+\left(\Delta P_{q q} \otimes g_{1}^{q}+\Delta P_{q g} \otimes g_{1}^{g}\right)(x)\right], \\
x g_{L}^{\perp q}\left(x, p_{T}^{2}\right) & =\frac{\alpha_{s}}{2 \pi^{2}} \frac{1}{2 \boldsymbol{p}_{T}^{2}}\left[\frac{L\left(\eta^{-1}\right)}{2} g_{1}^{q}(x)+\left(\Delta P_{q q}^{\prime} \otimes g_{1}^{q}+\Delta P_{q g}^{\prime} \otimes g_{1}^{g}\right)(x)\right]
\end{aligned}
$$

and

$$
\begin{aligned}
h_{1}^{q}\left(x, p_{T}^{2}\right) & =\frac{\alpha_{s}}{2 \pi^{2}} \frac{1}{p_{T}^{2}}\left[\frac{L\left(\eta^{-1}\right)}{2} h_{1}^{q}(x)-C_{F} h_{1}^{q}(x)+\left(\delta P_{q q} \otimes h_{1}^{q}\right)(x)\right], \\
x h_{T}^{\perp q}\left(x, p_{T}^{2}\right) & =\frac{\alpha_{s}}{2 \pi^{2}} \frac{1}{2 p_{T}^{2}}\left[\frac{L\left(\eta^{-1}\right)}{2} h_{1}^{q}(x)+\left(\delta P_{q q} \otimes h_{1}^{q}\right)(x)\right], \\
x h_{T}^{q}\left(x, p_{T}^{2}\right) & =-x h_{T}^{\perp q}\left(x, p_{T}^{2}\right),
\end{aligned}
$$




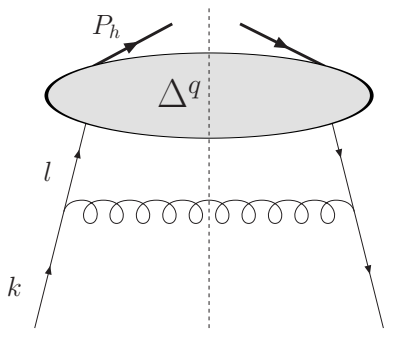

(a)

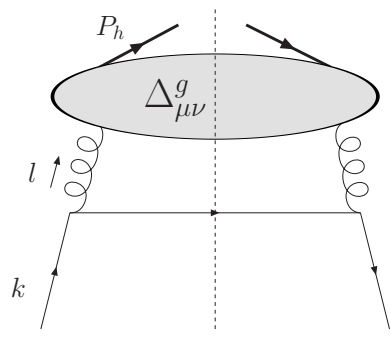

(b)

Figure 6: Diagrams for the calculation of the leading high- $k_{T}$ behavior of the quark-quark fragmentation correlator $\Delta\left(z, k_{T}\right)$ in axial gauge $A \cdot v=0$.

where $L\left(\eta^{-1}\right)$ is defined as in 3.13$)$. Here

$$
\delta P_{q q}(\hat{x})=C_{F}\left[\frac{2 \hat{x}}{(1-\hat{x})_{+}}+\frac{3}{2} \delta(1-\hat{x})\right]
$$

is the leading-order DGLAP splitting function for the transversity distribution [69], and the remaining splitting functions are given in (4.29) to (4.33). The chiral-odd quark distributions in (8.30) to (8.32) receive no contribution from (8.24) because chirality is conserved for the quark line in the graph of figure $5 \mathrm{~b}$.

The diagrams for the high- $p_{T}$ behavior of the antiquark correlation function $\Phi^{\bar{q}}\left(x, p_{T}\right)$ are obtained from those in figure 5 by reversing the direction of the fermion lines. The results have the form of (8.26) to (8.32), with identical splitting functions and with all quark distributions replaced by antiquark distributions.

\subsection{High- $k_{T}$ tails of fragmentation functions}

The calculation of the high-transverse-momentum tails of quark fragmentation functions proceeds in close analogy to the case of distribution functions. We nevertheless present the essential steps in this subsection, so as to show that no problems occur when going from a spacelike to a timelike situation.

The Feynman diagrams to be evaluated in $A \cdot v=0$ gauge are drawn in figure 6 . We first consider the quark-to-quark contribution of figure 6a. The analog of the starting expression (8.1) now reads

$$
\begin{aligned}
\left.\Delta^{q}\left(z, k_{T}\right)\right|_{\text {(国) }}= & \frac{4 \pi \alpha_{s}}{(2 \pi)^{3}} C_{F} \frac{1}{z} \int d k^{+} \int d l^{-} \delta\left((k-l)^{2}\right) \theta\left(k^{-}-l^{-}\right) \\
& \times\left. d^{\mu \nu}(k-l ; v) \frac{\not k}{k^{2}} \gamma_{\mu} \frac{\hat{z}}{z} \Delta_{2}^{q}\left(\frac{z}{\hat{z}}\right) \gamma_{\nu} \frac{\not k}{k^{2}}\right|_{l^{+}=0, \boldsymbol{l}_{T}=\mathbf{0}_{T}},
\end{aligned}
$$

where $k^{-}=P_{h}^{-} / z$ and $l^{-}=\hat{z} k^{-}$. The factors $1 / z$ and $\hat{z} / z$ in 8.34 arise from the definitions (5.9) and (5.21) of the fragmentation correlators. We perform the $k^{+}$-integration using

$$
\left.\delta\left((k-l)^{2}\right)\right|_{l^{+}=0, \boldsymbol{l}_{T}=\mathbf{0}_{T}}=\frac{1}{2 k^{-}(1-\hat{z})} \delta\left(k^{+}-\frac{\boldsymbol{k}_{T}^{2}}{2 k^{-}(1-\hat{z})}\right)
$$


and obtain

$$
\left.\Delta^{q}\left(z, k_{T}\right)\right|_{(\text {国 })}=\frac{\alpha_{s}}{(2 \pi)^{2}} C_{F} \frac{1}{z^{2} \boldsymbol{k}_{T}^{4}} \int_{z}^{1} \frac{d \hat{z}}{\hat{z}}(1-\hat{z}) d^{\mu \nu}(\bar{k}-\bar{l} ; v) \overline{\not k} \gamma_{\mu} \Delta_{2}^{q}\left(\frac{z}{\hat{z}}\right) \gamma_{\nu} \overline{\not k}
$$

with

$$
\begin{aligned}
\bar{k} & =\frac{\boldsymbol{k}_{T}^{2}}{2 k^{-}(1-\hat{z})} n_{+}+k^{-} n_{-}+k_{T}, \\
\bar{l} & =\hat{z} k^{-} n_{-} .
\end{aligned}
$$

The virtuality of the fragmenting quark

$$
\bar{k}^{2}=\frac{\hat{z} \boldsymbol{k}_{T}^{2}}{1-\hat{z}}
$$

is always timelike, in contrast to its counterpart $\bar{p}^{2}$ in the distribution correlator. For the calculation of the fragmentation correlator, it is useful to write the gauge vector as

$$
v=v^{+} n_{+}-\frac{2\left(P_{h}^{-}\right)^{2} v^{+}}{\zeta_{h}} n_{-}
$$

with

$$
\zeta_{h}=-\frac{\left(2 P_{h} \cdot v\right)^{2}}{v^{2}}=-\frac{2\left(P_{h}^{-}\right)^{2} v^{+}}{v^{-}} .
$$

In analogy to (8.10) and (8.11) we can then write

$$
(\bar{k}-\bar{l}) \cdot v=(\bar{k}-\bar{l})^{-} v^{+}-\frac{2\left(k^{-}\right)^{2}}{z^{-2} \zeta_{h}}(\bar{k}-\bar{l})^{+} v^{+}=\left[1-\hat{z}-\eta_{h} \frac{1}{1-\hat{z}}\right] k^{-} v^{+},
$$

where we have introduced

$$
\eta_{h}=\frac{\boldsymbol{k}_{T}^{2}}{z^{-2} \zeta_{h}}=-\frac{\boldsymbol{k}_{T}^{2}}{2\left(k^{-}\right)^{2}} \frac{v^{-}}{v^{+}}
$$

Taking the limit $\boldsymbol{k}_{T}^{2} \ll \zeta_{h}$ and following similar steps as in the previous subsection, we obtain

$$
\begin{aligned}
\left.\Delta^{q}\left(z, k_{T}\right)\right|_{\text {6 } 6 \mathrm{a})}=\frac{\alpha_{s}}{2 \pi^{2}} & C_{F} \frac{1}{z^{2} \boldsymbol{k}_{T}^{2}} \int_{z}^{1} \frac{d \hat{z}}{\hat{z}}\left\{\left[\frac{1}{(1-\hat{z})_{+}}+\frac{1}{2} \delta(1-\hat{z}) \ln \frac{1}{\eta_{h}}\right]\right. \\
& \left.\times \frac{(1-\hat{z})^{2}}{2 \boldsymbol{k}_{T}^{2}} d^{\mu \nu}\left(\bar{k}-\bar{l} ; n_{+}\right) \overline{k k} \gamma_{\mu} \Delta_{2}^{q}\left(\frac{z}{\hat{z}}\right) \gamma_{\nu} \overline{\not k}-\delta(1-\hat{z}) \Delta_{2}^{q}\left(\frac{z}{\hat{z}}\right)\right\}
\end{aligned}
$$

to leading order in $1 / k_{T}$, where as in the case of distribution functions the result is restricted to the twist-two and twist-three parts of the correlation function on the l.h.s. For the quark-to-gluon contribution, the diagram of figure $6 \mathrm{~b}$ gives

$$
\left.\Delta^{q}\left(z, k_{T}\right)\right|_{(\bar{G} \mathrm{~b})}=\frac{\alpha_{s}}{(2 \pi)^{2}} C_{F} \frac{1}{z^{2} \boldsymbol{k}_{T}^{4}} \int_{z}^{1} \frac{d \hat{z}}{\hat{z}}(1-\hat{z}) \Delta_{2}^{g, \mu \nu}\left(\frac{z}{\hat{z}}\right) \overline{\not k} \gamma_{\mu}(\overline{\not k}-\bar{\gamma}) \gamma_{\nu} \overline{\not k}
$$

where the twist-two part of the gluon fragmentation correlator is parameterized by just one function,

$$
\Delta_{2}^{g, \mu \nu}(z)=-\frac{z}{2 P_{h}^{-}} g_{T}^{\mu \nu} D_{1}^{g}(z)
$$


because we consider an unpolarized hadron. With the parameterization (5.9) of $\Delta^{q}\left(z, k_{T}\right)$ we obtain the high- $k_{T}$ behavior

$$
\begin{gathered}
D_{1}^{q}\left(z, k_{T}^{2}\right)=\frac{\alpha_{s}}{2 \pi^{2}} \frac{1}{z^{2} \boldsymbol{k}_{T}^{2}}\left[\frac{L\left(\eta_{h}^{-1}\right)}{2} D_{1}^{q}(z)-C_{F} D_{1}^{q}(z)+\left(D_{1}^{q} \otimes P_{q q}+D_{1}^{g} \otimes P_{g q}\right)(z)\right] \\
\frac{D^{\perp q}\left(z, k_{T}^{2}\right)}{z}=\frac{\alpha_{s}}{2 \pi^{2}} \frac{1}{z^{2} \boldsymbol{k}_{T}^{2}}\left\{\frac{L\left(\eta_{h}^{-1}\right)}{4} D_{1}^{q}(z)+\int_{z}^{1} \frac{d \hat{z}}{\hat{z}} D_{1}^{q}\left(\frac{z}{\hat{z}}\right) C_{F}\left[\frac{1}{(1-\hat{z})_{+}}+\frac{3}{4} \delta(1-\hat{z})\right]\right. \\
\left.\quad+\int_{z}^{1} \frac{d \hat{z}}{\hat{z}} D_{1}^{g}\left(\frac{z}{\hat{z}}\right) C_{F} \frac{2-\hat{z}}{\hat{z}}\right\}
\end{gathered}
$$

from (8.44) and (8.45). For $\tilde{D}^{\perp q}$ this implies

$$
\frac{\tilde{D}^{\perp q}\left(z, k_{T}^{2}\right)}{z}=-\frac{\alpha_{s}}{2 \pi^{2}} \frac{1}{2 z^{2} \boldsymbol{k}_{T}^{2}}\left[\frac{L\left(\eta_{h}^{-1}\right)}{2} D_{1}^{q}(z)-2 C_{F} D_{1}^{q}(z)+\left(D_{1}^{q} \otimes P_{q q}^{\prime}+D_{1}^{g} \otimes P_{g q}^{\prime}\right)(z)\right]
$$

according to its definition (5.71). Analogous results with the same kernels are obtained for the antiquark fragmentation functions $D_{1}^{\bar{q}}, D^{\perp \bar{q}}$, and $\tilde{D}^{\perp \bar{q}}$.

\subsection{Results for structure functions and their consequences}

We are now ready to compute the behavior of the structure functions $F_{U U, T}, F_{L L}, F_{U U}^{\cos \phi_{h}}$, and $F_{L L}^{\cos \phi_{h}}$ at intermediate transverse momentum. For $F_{U U, T}$ we start from the low- $q_{T}$ result (5.53), with the convolution defined in (5.75). Using the expansion (5.77) we have

$$
F_{U U, T}=\sum_{a} x e_{a}^{2}\left[f_{1}^{a}\left(x, q_{T}^{2}\right) \frac{D_{1}^{a}(z)}{z^{2}}+f_{1}^{a}(x) D_{1}^{a}\left(z, q_{T}^{2}\right)+f_{1}^{a}(x) \frac{D_{1}^{a}(z)}{z^{2}} U\left(q_{T}^{2}\right)\right]
$$

for $M \ll q_{T} \ll Q$. The high-transverse-momentum limits of $f_{1}^{a}\left(x, q_{T}^{2}\right)$ and $D_{1}^{a}\left(z, q_{T}^{2}\right)$ are respectively given in 8.26 ) and (8.47). For the corresponding limit of the soft factor one obtains

$$
U\left(q_{T}^{2}\right)=\frac{\alpha_{s} C_{F}}{\pi^{2}} \frac{1}{q_{T}^{2}}
$$

from [24], as we show in appendix C. Given that $2 P^{+} P_{h}^{-}=z Q^{2} / x$ up to mass corrections, the relations (8.9), (8.11) and (8.41), 8.43) imply

$$
\sqrt{\zeta \zeta_{h}}=\frac{z Q^{2}}{x}, \quad \sqrt{\eta \eta_{h}}=\frac{q_{T}^{2}}{Q^{2}},
$$

where in the second equation we have set $\boldsymbol{k}_{T}^{2}$ and $\boldsymbol{p}_{T}^{2}$ equal to $\boldsymbol{q}_{T}^{2}$, as appropriate for evaluating (8.50). Putting the above results together, we obtain

$$
\begin{aligned}
F_{U U, T}=\frac{\alpha_{s}}{2 \pi^{2}} & \frac{1}{z^{2} q_{T}^{2}} \sum_{a} x e_{a}^{2} \\
& \times\left\{\left[\frac{L\left(\eta^{-1}\right)}{2} f_{1}^{a}(x)-C_{F} f_{1}^{a}(x)+\left(P_{q q} \otimes f_{1}^{a}+P_{q g} \otimes f_{1}^{g}\right)(x)\right] D_{1}^{a}(z)\right. \\
& +f_{1}^{a}(x)\left[\frac{L\left(\eta_{h}^{-1}\right)}{2} D_{1}^{a}(z)-C_{F} D_{1}^{a}(z)+\left(D_{1}^{a} \otimes P_{q q}+D_{1}^{g} \otimes P_{g q}\right)(z)\right] \\
& \left.+2 C_{F} f_{1}^{a}(x) D_{1}^{a}(z)\right\}
\end{aligned}
$$




$$
\begin{gathered}
=\frac{1}{q_{T}^{2}} \frac{\alpha_{s}}{2 \pi^{2} z^{2}} \sum_{a} x e_{a}^{2}\left[f_{1}^{a}(x) D_{1}^{a}(z) L\left(\frac{Q^{2}}{q_{T}^{2}}\right)+f_{1}^{a}(x)\left(D_{1}^{a} \otimes P_{q q}+D_{1}^{g} \otimes P_{g q}\right)(z)\right. \\
\left.+\left(P_{q q} \otimes f_{1}^{a}+P_{q g} \otimes f_{1}^{g}\right)(x) D_{1}^{a}(z)\right]
\end{gathered}
$$

which is identical with the result (4.23) of the high- $q_{T}$ calculation. The same agreement has been found by Ji et al. [7], who used the low- $q_{T}$ factorization scheme specified in [26] instead of the original Collins-Soper scheme [24]. Note that the terms with $C_{F} f_{1}^{a}(x) D_{1}^{a}(z)$ cancel among the different contributions in (8.53). By virtue of (8.52) the dependence on the gauge parameters $\eta$ and $\eta_{h}$ also cancels, as it should. We remark that we obtain the same final result if we take a timelike vector $v$ instead of a spacelike one. Both $\eta$ and $\eta_{h}$ are then negative, and $L\left(\eta^{-1}\right)$ and $L\left(\eta_{h}^{-1}\right)$ are replaced by $L\left(-\eta^{-1}\right)$ and $L\left(-\eta_{h}^{-1}\right)$, so that they still add up to $2 L\left(Q^{2} / q_{T}^{2}\right)$. For $F_{L L}$ we obtain a result analogous to (8.53), with the parton distributions $f_{1}$ replaced by $g_{1}$ and the convolutions $P \otimes f_{1}$ by $\Delta P \otimes g_{1}$. This result exactly matches the expression (4.27) obtained in the high- $q_{T}$ calculation.

We now turn to the structure function $F_{U U}^{\cos \phi_{h}}$. According to (5.45) and (5.50) the terms with $h_{1}^{\perp}$ and $H_{1}^{\perp}$ in the low- $q_{T}$ expression (5.55) are power suppressed compared to the terms with $f^{\perp}$ and $\tilde{D}^{\perp}$ when $q_{T} \gg M$. For intermediate $q_{T}$ we therefore have

$$
F_{U U}^{\cos \phi_{h}}=-\frac{2 q_{T}}{Q} \sum_{a} x e_{a}^{2}\left[x f^{\perp a}\left(x, q_{T}^{2}\right) \frac{D_{1}^{a}(z)}{z^{2}}-f_{1}^{a}(x) \frac{\tilde{D}^{\perp a}\left(z, q_{T}^{2}\right)}{z}\right]
$$

at leading power and leading order in $\alpha_{s}$. In this case there is no leading contribution from the soft factor taken at large transverse momentum. Proceeding as we did in (5.78), one finds that the leading term in the expansion of $U\left(l_{T}^{2}\right)$ around $\boldsymbol{l}_{T}=-\boldsymbol{q}_{T}$ gives zero in the convolution (5.55) because it does not depend on a direction in the transverse plane, whereas the next terms in the expansion only give contributions that are power suppressed compared to those in (8.54). We therefore obtain the same result 8.54) if we omit the soft factor in the transverse-momentum convolution (5.75). Using the high-transversemomentum limits 8.27) and (8.49) of $f^{\perp a}\left(x, q_{T}^{2}\right)$ and $\tilde{D}^{\perp a}\left(z, q_{T}^{2}\right)$, we get

$$
\begin{aligned}
F_{U U}^{\cos \phi_{h}}= & -\frac{2 q_{T}}{Q} \frac{\alpha_{s}}{2 \pi^{2}} \frac{1}{2 z^{2} q_{T}^{2}} \sum_{a} x e_{a}^{2} \\
\times & \times\left\{\left[\frac{L\left(\eta^{-1}\right)}{2} f_{1}^{a}(x)+\left(P_{q q}^{\prime} \otimes f_{1}^{a}+P_{q g}^{\prime} \otimes f_{1}^{g}\right)(x)\right] D_{1}^{a}(z)\right. \\
& \left.\quad+f_{1}^{a}(x)\left[\frac{L\left(\eta_{h}^{-1}\right)}{2} D_{1}^{a}(z)-2 C_{F} D_{1}^{a}(z)+\left(D_{1}^{a} \otimes P_{q q}^{\prime}+D_{1}^{g} \otimes P_{g q}^{\prime}\right)(z)\right]\right\} \\
=- & \frac{1}{Q q_{T}} \frac{\alpha_{s}}{2 \pi^{2} z^{2}} \sum_{a} x e_{a}^{2}\left[f_{1}^{a}(x) D_{1}^{a}(z) L\left(\frac{Q^{2}}{q_{T}^{2}}\right)+f_{1}^{a}(x)\left(D_{1}^{a} \otimes P_{q q}^{\prime}+D_{1}^{a} \otimes P_{g q}^{\prime}\right)(z)\right. \\
& \left.\quad+\left(P_{q q}^{\prime} \otimes f_{1}^{a}+P_{q g}^{\prime} \otimes f_{1}^{g}\right)(x) D_{1}^{a}(z)-2 C_{F} f_{1}^{a}(x) D_{1}^{a}(z)\right], \quad(8.55)
\end{aligned}
$$

which is not identical to the high- $q_{T}$ result (4.25) because of the extra term $2 C_{F} f_{1}^{a}(x) D_{1}^{a}(z)$ in the brackets. The same situation is found for $F_{L L}^{\cos \phi_{h}}$, with $f_{1}$ replaced by $g_{1}$ and $P^{\prime} \otimes f_{1}$ by $\Delta P^{\prime} \otimes g_{1}$ 
This disagreement has important consequences. Since the leading terms in the high- $q_{T}$ and the low- $q_{T}$ calculation of $F_{U U}^{\cos \phi_{h}}$ have the same power behavior for $M \ll q_{T} \ll Q$, their explicit expressions in that region must agree if both of them are calculated correctly. This is clear, since both calculations give the same term of a double expansion in $M / q_{T}$ and $q_{T} / Q$, as given in (1.2) and (1.4). We have no reason to doubt the validity of the high$q_{T}$ result (4.25), which comes from a twist-two calculation in collinear factorization. The same holds for the high-transverse-momentum behavior of the functions $f^{\perp a}\left(x, q_{T}^{2}\right)$ and $\tilde{D}^{\perp a}\left(z, q_{T}^{2}\right)$ in (8.27) and (8.49). In contrast, the low- $q_{T}$ expression we used for $F_{U U}^{\cos \phi_{h}}$ is a twist-three result, for which no proof of factorization is available. To obtain the expression in (8.54) we have assumed that the tree-level result (5.55) can be generalized by taking over the convolution (5.75) established for the twist-two sector. The comparison of (8.55) with (4.25) implies that this assumption is incorrect.

Based on our finding, one may speculate how a correct twist-three factorization formula will look like if factorization can be established at that level. Simple modification of the soft factor $U\left(l_{T}^{2}\right)$ can obviously not yield agreement with the high- $q_{T}$ result since this factor does not appear in the limiting expression (8.54) for the reasons we explained above. The situation would be different if the soft factor were dependent on the direction of $\boldsymbol{l}_{T}$, which would require it to have a nontrivial structure in either Lorentz or Dirac space (through factors $l_{T}^{\mu}$ or $y_{T}$ ). Such a dependence would go beyond the eikonal approximation for the coupling of soft gluons to fast partons, which may be necessary at subleading order in $1 / Q$. We shall not pursue such speculations here. Clearly, the requirement to match the high- $q_{T}$ result (4.25) for $F_{U U}^{\cos \phi_{h}}$ at intermediate $q_{T}$ can be used as a consistency check for any framework that extends Collins-Soper factorization to the twist-three sector.

It is instructive to note that the low- and high- $q_{T}$ results disagree by a term proportional to $f_{1}^{a}(x) D_{1}^{a}(z)$, where neither the distribution nor the fragmentation function appears in a convolution over longitudinal momentum fractions. In the calculations of the previous subsections, such terms arise from configurations where a gluon has zero plus- or minus-momentum. The correct treatment of this phase space region is nontrivial already in proofs of factorization at the twist-two level [26, 27, so that it is not too surprising that this is where problems occur in the naive extension to twist three which we have explored.

At this point we return to the issue of transverse-momentum-resummation for $F_{U U}^{\cos \phi_{h}}$, which we have briefly discussed in section 3.3. We can now understand why the splitting functions $P_{q q}^{\prime}, P_{q g}^{\prime}$, and $P_{g q}^{\prime}$ in the high- $q_{T}$ result 4.25) are different from the usual DGLAP kernels. Up to $\delta$-function terms they describe the high-transverse-momentum behavior of $f^{\perp}$ and $\tilde{D}^{\perp}$, rather than the one of the more familiar functions $f_{1}$ and $D_{1}$. A corresponding remark applies to the $\cos \phi$ asymmetry in Drell-Yan production investigated in [9]. If a low$q_{T}$ factorization formula for these observables can be established, it should also allow one to adapt the original CSS procedure [1] for the resummation of large logarithms $\ln \left(Q^{2} / q_{T}^{2}\right)$ at next-to-leading logarithmic accuracy and beyond. From this point of view, resummation for $F_{U U}^{\cos 2 \phi_{h}}$ and its analogs in Drell-Yan production or $e^{+} e^{-}$annihilation appears rather daunting since it would require a formulation of low- $q_{T}$ factorization at twist-four level, extending the simple parton-model result in (6.15) and putting it on a rigorous footing. 


\section{Summary}

The description of semi-inclusive deep inelastic scattering with measured transverse momentum $q_{T}$ involves two theoretical frameworks: at low $q_{T}$ one has a factorized representation in terms of transverse-momentum-dependent distribution and fragmentation functions, whereas at high $q_{T}$ standard collinear factorization can be used. We have systematically analyzed the relation between the two descriptions at intermediate transverse momentum $M \ll q_{T} \ll Q$, where both are applicable. Depending on the specific observable, the leading terms in the two descriptions may or may not coincide.

Using dimensional analysis and Lorentz invariance, we have derived the general behavior at high $p_{T}$ for all transverse momentum-dependent parton distributions of twist two or three. The results, listed in eqs. (5.44) to (5.48), involve the convolution of collinear parton distributions with hard-scattering kernels, which in the simplest cases are closely related to the well-known DGLAP splitting functions. We have computed these kernels at leading order in $\alpha_{s}$ for those cases where the collinear distributions are of leading twist, obtaining the expressions (8.26) to 8.32). With these results and their analogs for transversemomentum-dependent fragmentation functions we could establish in eqs. (5.79) to (5.94) the power behavior for $M \ll q_{T} \ll Q$ of all SIDIS structure functions that appear in the low- $q_{T}$ description at twist-two or twist-three accuracy, allowing for arbitrary polarization of target and beam.

In the high- $q_{T}$ description at order $\alpha_{s}$ one finds a considerable simplification when taking the limit $q_{T} \ll Q$ : the expressions of the structure functions then involve a convolution of either the distribution or the fragmentation functions with hard-scattering kernels, whereas the other function is evaluated at the momentum fraction $x$ or $z$ fixed by the kinematics of the final state. For observables where the high- $q_{T}$ and low- $q_{T}$ calculations match, these kernels can be identified with the ones describing the high-transverse-momentum behavior of the functions appearing in the low- $q_{T}$ description. In such a situation one can use the procedure of Collins, Soper, and Sterman to resum large logarithms of $Q^{2} / q_{T}^{2}$ to all orders in perturbation theory. A prerequisite for this is that the power behavior of the observable in the low- and high- $q_{T}$ calculations must match. We have compared the corresponding powers for a wide range of observables, using our low- $q_{T}$ results (5.79) to (5.94) and their counterparts (6.1) to (6.13) for those structure functions that have been evaluated in the high- $q_{T}$ formulation. This comparison, compiled in table 2, is one of the main results of our work.

When the two formulations give the same power law at intermediate $q_{T}$ for a given observable, their explicit results must agree exactly because they describe the same term of a double expansion in $M / q_{T}$ and $q_{T} / Q$. This constitutes a nontrivial consistency check for both the low- and high- $q_{T}$ calculations. Confirming earlier results in the literature, we have verified that there is such an agreement for the unpolarized structure function $F_{U U, T}$, as well as for its analog $F_{L L}$ for longitudinal beam and target polarization. By contrast, the structure function $F_{U U, L}$ for longitudinal photon polarization only appears at twist four in the low- $q_{T}$ framework, where a complete result is not available. A simple calculation in the parton model gives a power behavior which in the intermediate region matches the one of 
the well-established high- $q_{T}$ result but fails to reproduce its exact form.

A more involved picture arises for azimuthal asymmetries, even in unpolarized scattering. At low $q_{T}$ the structure function $F_{U U}^{\cos 2 \phi_{h}}$ is expressed in terms of the Boer-Mulders function $h_{1}^{\perp}$ and the Collins fragmentation function $H_{1}^{\perp}$, both of which are chiral-odd, whereas the high- $q_{T}$ expression involves the usual unpolarized distribution and fragmentation functions $f_{1}$ and $D_{1}$, which are chiral-even. The two results thus describe different physical mechanisms, which is consistent with our finding that at intermediate $q_{T}$ they have a different power behavior. In this region, the two results may hence be added. In practice, some arbitrariness is involved in deciding what "intermediate" $q_{T}$ values are. We have shown that the sum of the high- $q_{T}$ and the low- $q_{T}$ expressions gives a valid approximation for $F_{U U}^{\cos 2 \phi_{h}}$ also at large $q_{T}$, where the low- $q_{T}$ result cannot be trusted but is power suppressed compared with the high- $q_{T}$ expression. The latter, however, fails to vanish in the limit $q_{T} \rightarrow 0$, as required by angular momentum conservation, and should hence not be used at low $q_{T}$. A more favorable observable in this respect is the $\cos 2 \phi_{h}$ asymmetry, i.e., the ratio of $F_{U U}^{\cos 2 \phi_{h}}$ and the $\phi_{h}$ independent part $F_{U U, T}+\varepsilon F_{U U, L}$ of the cross section. In this case, the sum (6.26) of the expressions calculated for low and high $q_{T}$ gives a consistent approximation for all transverse momenta, up to corrections of order $M^{2} / q_{T}^{2}$ and $q_{T}^{2} / Q^{2}$. The result of a parton-model calculation at low $q_{T}$, often referred to as Cahn effect, has the same property for $F_{U U}^{\cos 2 \phi_{h}}$ as it has for $F_{U U, L}$ : its power behavior agrees with the high- $q_{T}$ result in the intermediate region, but its explicit expression does not. The parton-model result may hence only be regarded as a partial estimate for the full but unknown twist-four correction to $F_{U U}^{\cos 2 \phi_{h}}$ at low $q_{T}$.

The description of the structure function $F_{U U}^{\cos \phi_{h}}$ is more problematic: at high $q_{T}$ it can be evaluated in collinear factorization at twist-two level, but at low $q_{T}$ it requires a twistthree calculation, for which transverse-momentum-dependent factorization at all orders in $\alpha_{s}$ has not been established. As a working hypothesis we have taken the well-established result of a tree-level calculation at low $q_{T}$ and assumed that the soft factor which explicitly appears in the factorization theorem for twist-two observables is also applicable at twist three. This leads to an expression that for intermediate $q_{T}$ agrees with the high- $q_{T}$ result in its power behavior and in the form of the hard-scattering kernels, except for a term proportional to $f_{1}(x) D_{1}(z)$. We find this partial agreement encouraging, but it does show that our candidate factorization formula at twist three is incorrect as it stands, and that a proper analysis will have to devote special attention to gluons with vanishing plus- or minus-momentum and to the precise form of soft factors. We emphasize that the correct description of $F_{U U}^{\cos \phi_{h}}$ at low $q_{T}$ is a prerequisite for applying the method of Collins, Soper, and Sterman to resum large logarithms of $Q^{2} / q_{T}^{2}$.

The structure function $F_{U T, T}^{\sin \left(\phi_{h}-\phi_{S}\right)}$ for a transversely polarized target presents a case where the low- $q_{T}$ calculation is of twist two, whereas the high- $q_{T}$ description is at the twistthree level. The explicit computations in [5-8] find exact agreement of the two descriptions at intermediate $q_{T}$ and thus validate both frameworks. One may expect that the same is true for $F_{U T}^{\sin \left(\phi_{h}+\phi_{S}\right)}$, which at low $q_{T}$ is described in terms of the Collins effect.

Observables that are integrated over $q_{T}$ are at times preferable to differential ones from 
an experimental point of view. We have shown that some of them have the added virtue of admitting a relatively simple description at the theory level, both for the complexity of the expressions and for the number of distribution and fragmentation functions on which they depend. With the power-counting behavior listed in table 2 one can readily determine to which region of $q_{T}$ a given integrated observable is primarily sensitive. The results for selected observables are given in table 3. We find for instance that $\left\langle\left\langle F_{U U}^{\cos 2 \phi_{h}}\right\rangle\right\rangle$ and $\left\langle\left\langle F_{U T}^{\sin \phi_{S}}\right\rangle\right\rangle$ receive leading contributions from both low and high $q_{T}$. The integrated structure function $\left\langle\left\langle F_{U U}^{\cos \phi_{h}}\right\rangle\right\rangle$ is dominated by large $q_{T}$, with contributions from the low- $q_{T}$ region being suppressed by $M / Q$. Conversely, both $\left\langle\left\langle F_{U T, T}^{\sin \left(\phi_{h}-\phi_{S}\right)}\right\rangle\right\rangle$ and $\left\langle\left\langle F_{U T}^{\sin \left(\phi_{h}+\phi_{S}\right)}\right\rangle\right\rangle$ receive their dominant contributions from low $q_{T}$, whereas the high- $q_{T}$ domain is suppressed by $M / Q$. They are hence sensitive to the Sivers function in the first case, and to the transversity distribution and the Collins fragmentation function in the second. A suppression by $M / Q$ may, however, not be sufficient to simply neglect the corresponding contributions in an analysis at experimentally achievable values of $Q$.

A theoretically cleaner access to the high- $q_{T}$ region is through observables that are weighted with an appropriate power of $q_{T} / M$. We find in particular that $\left\langle\left\langle\left(q_{T} / M\right) F_{U U}^{\cos \phi_{h}}\right\rangle\right\rangle$, $\left\langle\left\langle\left(q_{T} / M\right)^{2} F_{U U}^{\cos 2 \phi_{h}}\right\rangle\right\rangle$, and $\left\langle\left\langle\left(q_{T} / M\right)^{2} F_{U T}^{\sin \phi_{S}}\right\rangle\right\rangle$ can be evaluated from the high- $q_{T}$ results alone, up to corrections of order $M^{2} / Q^{2}$, and that at the same accuracy one can extend the integration down to $q_{T}=0$. This leads to simple expressions, similar to the one for the integrated longitudinal structure function $\left\langle\left\langle F_{U U, L}\right\rangle\right\rangle$. The observables $\left\langle\left\langle\left(q_{T} / M\right) F_{U U}^{\cos \phi_{h}}\right\rangle\right\rangle$ and $\left\langle\left\langle\left(q_{T} / M\right)^{2} F_{U U}^{\cos 2 \phi_{h}}\right\rangle\right\rangle$ are sensitive to the twist-two functions $f_{1}$ and $D_{1}$ and may for instance be useful for separating the contributions from different quark flavors, serving as complements to $\left\langle\left\langle F_{U U, T}\right\rangle\right\rangle$. In contrast, $\left\langle\left\langle\left(q_{T} / M\right)^{2} F_{U T}^{\sin \phi_{S}}\right\rangle\right\rangle$ is sensitive to distribution and fragmentation functions of twist three.

The weighted structure functions $\left\langle\left\langle\left(q_{T} / M\right) F_{U T, T}^{\sin \left(\phi_{h}-\phi_{S}\right)}\right\rangle\right\rangle$ and $\left\langle\left\langle\left(q_{T} / M\right) F_{U T}^{\sin \left(\phi_{h}+\phi_{S}\right)}\right\rangle\right\rangle$ play a special role in this context. They receive leading-power contributions from both low and high $q_{T}$ and, as already pointed out in [12], lead to a deconvolution of the transversemomentum integrals in the low- $q_{T}$ result. We argued that they should permit a description in terms of collinear functions of twist two and three, defined in the standard $\overline{\mathrm{MS}}$ scheme. In this description, the low- $q_{T}$ expression gives the Born-level result, whereas the high- $q_{T}$ calculation of [43, 44, 8] gives part of the $\alpha_{s}$ corrections. If completed, such a description would provide a full NLO result in $\alpha_{s}$ and be an extension to twist-three level of the standard NLO calculation for $\left\langle\left\langle F_{U U, T}\right\rangle\right\rangle$ within collinear factorization at twist-two accuracy. The leading-order expressions $(7.9)$ and $(7.10)$ for the weighted structure functions are analogs of the familiar tree-level formula $\left\langle\left\langle F_{U U, T}\right\rangle\right\rangle=\sum_{a} x e_{a}^{2} f_{1}^{a}(x) D_{1}^{a}(z)$. These expressions receive corrections from the high- $q_{T}$ region which are of leading power but suppressed by $\alpha_{s}$.

Let us finally remark that the results we have discussed here carry over to the analogous observables in the Drell-Yan process and in $e^{+} e^{-}$annihilation. The SIDIS structure functions $F_{U U}^{\cos \phi_{h}}$ and $F_{U U}^{\cos 2 \phi_{h}}$ correspond for instance to the $\cos \phi$ and $\cos 2 \phi$ asymmetries in the angular distribution of the lepton pair in unpolarized Drell-Yan production, which have been measured [70] and given rise to several theoretical investigations, see e.g. the references in [9]. Furthermore, $F_{U U}^{\cos 2 \phi_{h}}$ corresponds to a $\cos 2 \phi$ asymmetry for two-pion production in $e^{+} e^{-}$annihilation, which has been measured by BELLE [71] and provides 
the possibility for an independent determination of the Collins fragmentation function [72]. For a reliable extraction, our discussion of matching low- and high-transverse-momentum contributions should be of relevance.

\section{Acknowledgments}

It is our pleasure to acknowledge valuable discussions with John Collins and Werner Vogelsang. We thank Jochen Bartels for helpful remarks on the manuscript. This research is part of the Integrated Infrastructure Initiative "Hadron Physics" of the European Union under contract number RII3-CT-2004-506078. The work of M.D. is partially supported by the Helmholtz Association, contract number VH-NG-004, and the work of A.B. is partially supported by the SFB "Particles, Strings and the Early Universe". The Feynman diagrams in this paper were drawn using JaxoDraw [73].

\section{A. Distribution functions at high $p_{T}$ : Feynman versus axial gauge}

Our calculation in section 8.1 is done in axial gauge $A \cdot v=0$. Let us see how the same calculation proceeds in Feynman gauge. In this case one must explicitly take into account the gauge link $\mathcal{U}$ in the definition (5.3) of the correlation function $\Phi\left(x, p_{T}\right)$, which consists of sections pointing along $v$ and a transverse section at infinity. The detailed path of the gauge link reflects important physics, as shown for instance in [13, 47- 50, 54].

Let us consider the correlation function $\Phi^{[+]}\left(x, p_{T}\right)$ relevant for SIDIS, whose gauge link $\mathcal{U}^{+}$is closed at $a^{-}=+\infty$. To evaluate the quark-to-quark contribution to the high$p_{T}$ behavior of $\Phi\left(x, p_{T}\right)$ at leading order in $1 / p_{T}$, one has to take into account the four diagrams shown in figure 0 . The graphs with eikonal lines are due to gluons coupling to the gauge link in the operator $\bar{\psi}_{j}(0) \mathcal{U}_{(0, \xi)} \psi_{i}(\xi)$. The corresponding Feynman rules read 24, 55]

$$
\mathfrak{g}_{a, \mu}=i g t^{a} v^{\mu}, \quad \quad \underline{l}=\frac{i}{l \cdot v+i \epsilon},
$$

where the sign of $i \epsilon$ for the eikonal line corresponds to a gauge link pointing to $a^{-}=+\infty$ if one takes $v^{-}>0$. In cut diagrams one must take the conjugate of these expressions for vertices and propagators on the right of the final-state cut (indicated by the dashed lines in figure (7).

After performing the integration over $p^{-}$using the $\delta$ function in (8.3), we have for the respective diagrams

$$
\begin{aligned}
& \left.\Phi^{[+] q}\left(x, p_{T}\right)\right|_{\text {(耳) }}=-\frac{\alpha_{s}}{(2 \pi)^{2}} C_{F} \int \frac{d \hat{x}}{\hat{x}(1-\hat{x})} g^{\mu \nu} \frac{\not p}{\bar{p}^{2}} \gamma_{\nu} \Phi_{2}^{q}\left(\frac{x}{\hat{x}}\right) \gamma_{\mu} \frac{\not p}{\bar{p}^{2}}, \\
& \left.\Phi^{[+] q}\left(x, p_{T}\right)\right|_{(\mathbf{b} \mathrm{b})}=-\frac{\alpha_{s}}{(2 \pi)^{2}} C_{F} \int \frac{d \hat{x}}{\hat{x}(1-\hat{x})} \frac{\not p}{\bar{p}^{2}} \psi \Phi_{2}^{q}\left(\frac{x}{\hat{x}}\right) \frac{1}{(\bar{l}-\bar{p}) \cdot v-i \epsilon}, \\
& \left.\Phi^{[+] q}\left(x, p_{T}\right)\right|_{\left(g_{c}\right)}=-\frac{\alpha_{s}}{(2 \pi)^{2}} C_{F} \int \frac{d \hat{x}}{\hat{x}(1-\hat{x})} \frac{1}{(\bar{l}-\bar{p}) \cdot v+i \epsilon} \Phi_{2}^{q}\left(\frac{x}{\hat{x}}\right) \psi \frac{\not p}{\bar{p}^{2}}, \\
& \left.\Phi^{[+] q}\left(x, p_{T}\right)\right|_{\left(\mathrm{g}_{\mathrm{d}}\right)}=-\frac{\alpha_{s}}{(2 \pi)^{2}} C_{F} \int \frac{d \hat{x}}{\hat{x}(1-\hat{x})} \Phi_{2}^{q}\left(\frac{x}{\hat{x}}\right) \frac{v^{2}}{[(\bar{l}-\bar{p}) \cdot v+i \epsilon][(\bar{l}-\bar{p}) \cdot v-i \epsilon]}
\end{aligned}
$$




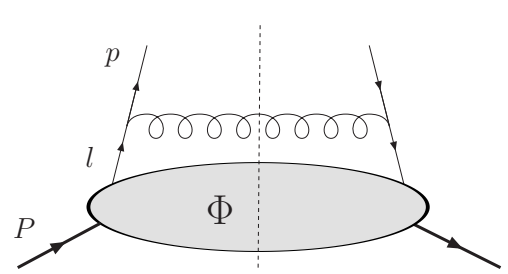

(a)

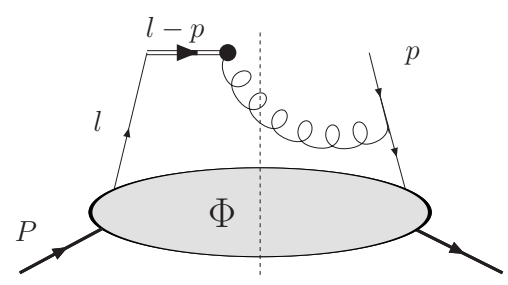

(c)

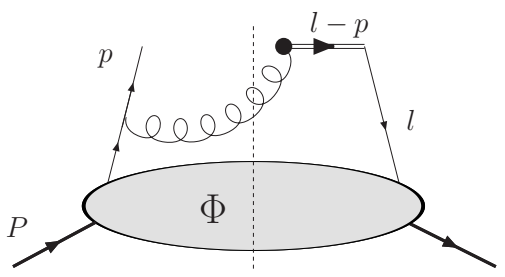

(b)

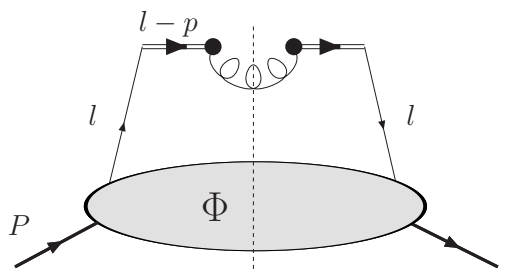

(d)

Figure 7: Diagrams for the calculation of the leading high- $p_{T}$ behavior of the quark-quark correlator $\Phi\left(x, p_{T}\right)$ in Feynman gauge.

with $\bar{p}$ and $\bar{l}$ given in (8.5) and (8.6). Since $\bar{p}^{2}$ is always spacelike according to (8.7), we have omitted the $i \epsilon$ in the quark propagators.

In the calculation using axial gauge only the first of the four diagrams contributes, but instead of $g^{\mu \nu}$ in (A.2) we then have to take

$$
-d^{\mu \nu}(\bar{l}-\bar{p} ; v)=g^{\mu \nu}-\frac{(\bar{l}-\bar{p})^{\mu} v^{\nu}+(\bar{l}-\bar{p})^{\nu} v^{\mu}}{(\bar{l}-\bar{p}) \cdot v}+\frac{(\bar{l}-\bar{p})^{\mu}(\bar{l}-\bar{p})^{\nu}}{[(\bar{l}-\bar{p}) \cdot v]^{2}} v^{2} .
$$

Each term in (A.6) corresponds to one of the four diagrams in the Feynman gauge calculation. The correspondence between the first term and diagram $7 \mathrm{a}$ in Feynman gauge is trivial. To establish the correspondence between the second term and diagram $7 \mathrm{~b}$ we use that

$$
\psi \Phi_{2}^{q}(\bar{\gamma}-\not \supset) \frac{\not p}{\bar{p}^{2}}=\psi \Phi_{2}^{q} \bar{\gamma} \frac{\not p}{\bar{p}^{2}}-\psi \Phi_{2}^{q}=-\psi \Phi_{2}^{q},
$$

where in the second step we have used that the twist-two part of the collinear quark correlator satisfies $\Phi_{2}^{q} \bar{l}=\Phi_{2}^{q} \not n_{+} l^{+}=0$. In an analogous way one establishes the correspondence between the last two terms in (A.6) with the respective contributions of diagrams $7 \mathrm{c}$ and $7 \mathrm{~d}$ in Feynman gauge.

A mismatch between the expressions in (A.3) to (A.5) and the calculation in axial gauge is, however, the different treatment of the singularities at $(\bar{l}-\bar{p}) \cdot v=0$. The principal value prescription we employed when using the spacelike axial gauge of the original CollinsSoper paper [24] differs from the $i \epsilon$ prescription for the different terms in the Feynman gauge calculation, which arises from the structure of the Wilson line $\mathcal{U}^{+}$in the correlation function. We note that the integral in (A.5) is actually not well defined as it stands, since the double pole at $(\bar{l}-\bar{p}) \cdot v=0$ is pinched. The contribution from such unphysical 
poles must be absent in the physical cross section and should hence cancel between the distribution function, the fragmentation function, and the soft factor in the factorization formula (3.22). How to implement this by regulating the individual factors has so far not been addressed in the literature. It is also currently unknown if and how the principalvalue prescription in axial gauge can be implemented in terms of Wilson lines for Feynman gauge. The discussion in [47] is for a light-cone rather than an axial gauge and hence does not contain the problematic term (A.5).

We remark that corresponding problems did not appear in the Feynman gauge calculation of $\mathrm{Ji}$ et al. [26], where the vector $v$ was chosen to be timelike. If we do the same in our context, then the calculations in Feynman and axial gauge exactly coincide. This is because $(\bar{l}-\bar{p}) \cdot v$ remains positive in $(\overline{\text { A.3 }})$ to (A.5) according to $(8.10)$, so that the singularity at $(\bar{l}-\bar{p}) \cdot v=0$ is not reached in the loop integral. As a consequence, the particular regularization of the axial-gauge propagator does not influence our results of section 8 if we take $v$ timelike. Likewise, there is then no contribution from transverse segments of the gauge link at infinity, which involve a $\delta$ function in $(\bar{l}-\bar{p}) \cdot v$. This is not implausible, since the distribution functions considered in section 8 are $T$-even and must in particular be the same for the gauge links $\mathcal{U}^{+}$and $\mathcal{U}^{-}$.

\section{B. Integrated distribution functions and transverse-momentum cutoff}

In this appendix we derive eq. (5.17), which relates two different ways of regularizing the integral over the transverse-momentum-dependent distribution $f_{1}\left(x, p_{T}^{2}\right)$. More precisely we show that with $\mu=b_{0} / b$ one has

$$
\int d^{2} \boldsymbol{p} e^{i \boldsymbol{b} \cdot \boldsymbol{p}} f\left(\boldsymbol{p}^{2}\right)=\pi \int_{0}^{\infty} d p^{2} J_{0}(b p) f\left(p^{2}\right)=\pi \int_{0}^{\mu^{2}} d p^{2} f\left(p^{2}\right)+\mathcal{O}\left(b^{2} \lambda^{2}\right)
$$

for any function that can be expanded as

$$
f\left(p^{2}\right)=\frac{c_{2}}{p^{2}}+\frac{c_{4}}{p^{4}}+\frac{c_{6}}{p^{6}}+\cdots
$$

for $p>\lambda$, where $c_{2}, c_{4}, c_{6}$, etc. are constants. The intermediate scale $\lambda$ can be taken just large enough for $(\mathrm{B} .2)$ to be valid, since corrections going for instance like $M / \lambda$ do not appear. The power corrections in (B.1) are understood as up to logarithms in $b^{2} \lambda^{2}$. For ease of notation we have written $p=|\boldsymbol{p}|$ and omitted the subscript $T$.

To establish (B.1) we split the integrals into the regions $p<\lambda$ and $p>\lambda$. In the first region we can write

$$
\int_{0}^{\lambda^{2}} d p^{2} J_{0}(b p) f\left(p^{2}\right)=\int_{0}^{\lambda^{2}} d p^{2} f\left(p^{2}\right)+\mathcal{O}\left(b^{2} \lambda^{2}\right),
$$

using that the Bessel function admits a Taylor expansion $J_{0}(x)=1-\frac{1}{4} x^{2}+\cdots$ in even powers of $x$. In the region $p>\lambda$ we make use of the expansion (B.2). Focusing first on the $1 / p^{2}$ term, we write

$$
\int_{\lambda^{2}}^{\infty} \frac{d p^{2}}{p^{2}} J_{0}(b p)=2 \int_{b \lambda}^{\infty} \frac{d x}{x} J_{0}(x)=-2 \ln (b \lambda) J_{0}(b \lambda)+2 \int_{b \lambda}^{\infty} d x \ln x J_{1}(x),
$$


where in the second step we have integrated by parts. We now use that $\int_{0}^{\infty} d x \ln x J_{1}(x)=$ $\ln 2-\gamma_{E}$ and $\int_{0}^{y} d x \ln x J_{1}(x) \sim y^{2}$ for $y \rightarrow 0$, where the latter relation holds because $J_{1}(x) \sim x$ for $x \rightarrow 0$. Recalling that $b_{0}=2 e^{-\gamma_{E}}$ we obtain

$$
\int_{\lambda^{2}}^{\infty} \frac{d p^{2}}{p^{2}} J_{0}(b p)=2\left[\ln \left(2 e^{-\gamma_{E}}\right)-\ln (b \lambda)\right]+\mathcal{O}\left(b^{2} \lambda^{2}\right)=\int_{\lambda^{2}}^{\mu^{2}} \frac{d p^{2}}{p^{2}}+\mathcal{O}\left(b^{2} \lambda^{2}\right) .
$$

For the $1 / p^{4}$ term in $(\overline{B .2})$ we use again integration by parts to write

$$
\int_{\lambda^{2}}^{\infty} \frac{d p^{2}}{p^{4}} J_{0}(b p)=2 b^{2} \int_{b \lambda}^{\infty} \frac{d x}{x^{3}} J_{0}(x)=\frac{J_{0}(b \lambda)}{\lambda^{2}}-b^{2} \int_{b \lambda}^{\infty} \frac{d x}{x^{2}} J_{1}(x) .
$$

Since the integrand in the last term behaves like $1 / x$ for $x \rightarrow 0$, we have

$$
\int_{\lambda^{2}}^{\infty} \frac{d p^{2}}{p^{4}} J_{0}(b p)=\frac{1}{\lambda^{2}}+\mathcal{O}\left(b^{2} \lambda^{2}\right)=\int_{\lambda^{2}}^{\mu^{2}} \frac{d p^{2}}{p^{4}}+\mathcal{O}\left(b^{2} \lambda^{2}\right) .
$$

A similar argument can be given for terms going like $1 / p^{2 n}$ with $n>2$, which completes the proof of (B.1).

As an illustration of our result let us consider the simple form $f\left(p^{2}\right)=1 /\left(p^{2}+\lambda^{2}\right)$. The relevant integrals then are

$$
\int_{0}^{\infty} \frac{d p^{2}}{p^{2}+\lambda^{2}} J_{0}(b \lambda)=2 K_{0}(b \lambda), \quad \int_{0}^{\mu^{2}} \frac{d p^{2}}{p^{2}+\lambda^{2}}=\ln \left(1+\frac{\mu^{2}}{\lambda^{2}}\right) .
$$

With the behavior $K_{0}(x)=-\ln x+\ln b_{0}+\mathcal{O}\left(x^{2}\right)$ of the modified Bessel function at small $x$ one readily finds that the relation $(\overline{B .1})$ is satisfied.

As we have seen in section 8.1, a logarithmic factor $\ln \left(\zeta / p^{2}\right)$ appears in the explicit calculation for the high- $p_{T}$ behavior of distribution functions at order $\alpha_{s}$. One can easily repeat the above arguments for the case where the $1 / p^{2}$ term in (B.2) is multiplied by $\ln p^{2}$ and the subleading terms by some power of $\ln p^{2}$. Using that $\int_{0}^{\infty} d x(\ln x)^{2} J_{1}(x)=$ $\left(\ln 2-\gamma_{E}\right)^{2}$ one finds that (B.1) holds without modification also in this case.

\section{One-loop expression of the soft factor}

In this appendix we show how to obtain the momentum-space expression (8.51) of the soft factor in the Collins-Soper factorization formula 24]. The corresponding expression in $b$-space is given in eq. (7.22) of [24]. With our definition (3.7) we obtain $U\left(l_{T}^{2}\right)$ from this by setting $\varepsilon=0$ and omitting $\int d^{2} \boldsymbol{l}_{T} e^{i \boldsymbol{b} \cdot \boldsymbol{l}_{T}}$. The result is

$$
\begin{aligned}
U\left(l_{T}^{2}\right) & =-4 C_{F} \alpha_{s} \int \frac{d l^{+} d l^{-}}{(2 \pi)^{2}} \frac{\delta\left(l^{2}\right) \theta\left(l^{+}\right)}{l^{+} l^{-}} d^{+-}(l ; v) \\
& =4 C_{F} \alpha_{s} \int \frac{d l^{+} d l^{-}}{(2 \pi)^{2}} \delta\left(2 l^{+} l^{-}-\boldsymbol{l}_{T}^{2}\right) \theta\left(l^{+}\right) \frac{v^{2}}{(l \cdot v)^{2}} \\
& =\frac{C_{F} \alpha_{s}}{\pi^{2}} \mathrm{PV} \int_{0}^{\infty} d l^{+} \frac{4 l^{+} v^{-} / v^{+}}{\left[2\left(l^{+}\right)^{2} v^{-} / v^{+}+\boldsymbol{l}_{T}^{2}\right]^{2}}=\frac{C_{F} \alpha_{s}}{\pi^{2}} \frac{1}{\boldsymbol{l}_{T}^{2}},
\end{aligned}
$$


where on the last line we have indicated that for a spacelike gauge vector we need the principal value prescription to regulate the integral, given that $v^{-} / v^{+}<0$. In accordance with our footnote on page 8 , the result of the integration is independent of $v$. Fourier transforming the result (C.1) to $b$-space in $2-\varepsilon$ transverse dimensions, we obtain

$$
\frac{C_{F} \alpha_{s}}{\pi^{2}} \mu^{\varepsilon} \int \frac{d^{2-\varepsilon} \boldsymbol{l}_{T}}{(2 \pi)^{-\varepsilon}} e^{i \boldsymbol{l}_{T} \cdot \boldsymbol{b}} \frac{1}{\boldsymbol{l}_{T}^{2}}=-\frac{C_{F} \alpha_{s}}{\pi}\left[\ln \left(\mu^{2} b^{2} \pi e^{\gamma}\right)+\frac{2}{\varepsilon}\right]
$$

in agreement with eq. (7.23) in [24].

\section{References}

[1] J.C. Collins, D.E. Soper and G. Sterman, Transverse momentum distribution in Drell-Yan pair and $W$ and $Z$ boson production, Nucl. Phys. B 250 (1985) 199.

[2] R.N. Cahn, Azimuthal dependence in leptoproduction: a simple parton model calculation, Phys. Lett. B 78 (1978) 269.

[3] R.N. Cahn, Critique of parton model calculations of azimuthal dependence in leptoproduction, Phys. Rev. D 40 (1989) 3107.

[4] H. Georgi and H.D. Politzer, Clean tests of QCD in $\mu p$ scattering, Phys. Rev. Lett. 40 (1978) 3 .

[5] X. Ji, J.-W. Qiu, W. Vogelsang and F. Yuan, A unified picture for single transverse-spin asymmetries in hard processes, Phys. Rev. Lett. 97 (2006) 082002 hep-ph/0602239.

[6] X. Ji, J.-W. Qiu, W. Vogelsang and F. Yuan, Single transverse-spin asymmetry in Drell-Yan production at large and moderate transverse momentum, Phys. Rev. D 73 (2006) 094017 hep-ph/0604023.

[7] X. Ji, J.-W. Qiu, W. Vogelsang and F. Yuan, Single-transverse spin asymmetry in semi-inclusive deep inelastic scattering, Phys. Lett. B 638 (2006) 178 hep-ph/0604128.

[8] Y. Koike, W. Vogelsang and F. Yuan, On the relation between mechanisms for single-transverse-spin asymmetries, Phys. Lett. B 659 (2008) 878 [arXiv:0711.0636.

[9] D. Boer and W. Vogelsang, Drell-Yan lepton angular distribution at small transverse momentum, Phys. Rev. D 74 (2006) 014004 hep-ph/0604177.

[10] E.L. Berger, J.-W. Qiu and R.A. Rodriguez-Pedraza, Transverse momentum dependence of the angular distribution of the Drell-Yan process, Phys. Rev. D 76 (2007) 074006 arXiv:0708.0578].

[11] P.J. Mulders and R.D. Tangerman, The complete tree-level result up to order $1 / Q$ for polarized deep-inelastic leptoproduction, Nucl. Phys. B 461 (1996) 197 [Erratum ibid. B 484 (1997) 538] hep-ph/9510301.

[12] D. Boer and P.J. Mulders, Time-reversal odd distribution functions in leptoproduction, Phys. Rev. D 57 (1998) 5780 hep-ph/9711485.

[13] D. Boer, P.J. Mulders and F. Pijlman, Universality of T-odd effects in single spin and azimuthal asymmetries, Nucl. Phys. B 667 (2003) 201 hep-ph/0303034.

[14] A. Bacchetta et al., Semi-inclusive deep inelastic scattering at small transverse momentum, JHEP 02 (2007) 093 hep-ph/0611265. 
[15] L.P. Gamberg, D.S. Hwang, A. Metz and M. Schlegel, Light-cone divergence in twist-3 correlation functions, Phys. Lett. B 639 (2006) 508 hep-ph/0604022.

[16] European Muon collaboration, M. Arneodo et al., Measurement of hadron azimuthal distributions in deep inelastic muon proton scattering, Z. Physik C 34 (1987) 277;

E665 collaboration, M.R. Adams et al., Perturbative QCD effects observed in $490 \mathrm{GeV}$ deep inelastic muon scattering, Phys. Rev. D 48 (1993) 5057;

ZEUS collaboration, M. Derrick et al., Inclusive charged particle distributions in deep inelastic scattering events at HERA, Z. Physik C 70 (1996) 1 hep-ex/9511010;

H1 collaboration, C. Adloff et al., Measurement of charged particle transverse momentum spectra in deep inelastic scattering, Nucl. Phys. B 485 (1997) 3 hep-ex/9610006;

E665 collaboration, M.R. Adams et al., Inclusive single-particle distributions and transverse momenta of forward produced charged hadrons in $\mu p$ scattering at $470 \mathrm{GeV}$, Z. Physik $\mathbf{C ~} \mathbf{7 6}$ (1997) 441;

ZEUS collaboration, J. Breitweg et al., Measurement of multiplicity and momentum spectra in the current and target regions of the Breit frame in deep inelastic scattering at HERA, Eur. Phys. J. C 11 (1999) 251 hep-ex/9903056;

$\mathrm{H}$. Mkrtchyan et al., Transverse momentum dependence of semi-inclusive pion production, Phys. Lett. B 665 (2008) 20 arXiv:0709.3020.

[17] ZEUS collaboration, J. Breitweg et al., Measurement of azimuthal asymmetries in deep inelastic scattering, Phys. Lett. B 481 (2000) 199 hep-ex/0003017;

ZEUS collaboration, S. Chekanov et al., Measurement of azimuthal asymmetries in neutral current deep inelastic scattering at HERA, Eur. Phys. J. C 51 (2007) 289 hep-ex/0608053.

[18] CLAS collaboration, H. Avakian et al., Measurement of beam-spin asymmetries for deep inelastic $\pi^{+}$electroproduction, Phys. Rev. D 69 (2004) 112004 hep-ex/0301005;

HERMES collaboration, A. Airapetian et al., Beam-spin asymmetries in the azimuthal distribution of pion electroproduction, Phys. Lett. B 648 (2007) 164 hep-ex/0612059.

[19] HERMES collaboration, A. Airapetian et al., Observation of a single-spin azimuthal asymmetry in semi-inclusive pion electro-production, Phys. Rev. Lett. 84 (2000) 4047 hep-ex/9910062]; Single-spin azimuthal asymmetries in electroproduction of neutral pions in semi-inclusive deep-inelastic scattering, Phys. Rev. D 64 (2001) 097101 hep-ex/0104005; Measurement of single-spin azimuthal asymmetries in semi-inclusive electroproduction of pions and kaons on a longitudinally polarised deuterium target, Phys. Lett. B 562 (2003) 182 hep-ex/0212039; Subleading-twist effects in single-spin asymmetries in semi-inclusive deep-inelastic scattering on a longitudinally polarized hydrogen target, Phys. Lett. B 622 (2005) 14 hep-ex/0505042.

[20] HERMES collaboration, A. Airapetian et al., Single-spin asymmetries in semi-inclusive deep-inelastic scattering on a transversely polarized hydrogen target, Phys. Rev. Lett. 94 (2005) 012002 hep-ex/0408013;

HERMES collaboration, M. Diefenthaler, HERMES measurements of Collins and Sivers asymmetries from a transversely polarised hydrogen target, arXiv:0706.2242.

[21] COMPASS collaboration, V.Y. Alexakhin et al., First measurement of the transverse spin asymmetries of the deuteron in semi-inclusive deep inelastic scattering, Phys. Rev. Lett. 94 (2005) 202002 hep-ex/0503002;

COMPASS collaboration, E.S. Ageev et al., A new measurement of the Collins and Sivers asymmetries on a transversely polarised deuteron target, Nucl. Phys. B 765 (2007) 31 hep-ex/0610068; 
COMPASS collaboration, A. Kotzinian, Beyond Collins and Sivers: further measurements of the target transverse spin-dependent azimuthal asymmetries in semi-inclusive DIS from COMPASS, arXiv:0705.2402.

[22] U. D'Alesio and F. Murgia, Azimuthal and single spin asymmetries in hard scattering processes, arXiv:0712.4328.

[23] A. Bacchetta, U. D'Alesio, M. Diehl and C.A. Miller, Single-spin asymmetries: the Trento conventions, Phys. Rev. D 70 (2004) 117504 hep-ph/0410050.

[24] J.C. Collins and D.E. Soper, Back-to-back jets in QCD, Nucl. Phys. B 193 (1981) 381 [Erratum ibid. B 213 (1983) 545].

[25] J.C. Collins, What exactly is a parton density?, Acta Phys. Polon. B34 (2003) 3103 hep-ph/0304122.

[26] X.-D. Ji, J.-P. Ma and F. Yuan, QCD factorization for semi-inclusive deep-inelastic scattering at low transverse momentum, Phys. Rev. D 71 (2005) 034005 hep-ph/0404183.

[27] J.C. Collins, T.C. Rogers and A.M. Stasto, Fully unintegrated parton correlation functions and factorization in lowest order hard scattering, Phys. Rev. D 77 (2008) 085009 arXiv:0708.2833.

[28] P. Nadolsky, D.R. Stump and C.P. Yuan, Semi-inclusive hadron production at HERA: the effect of QCD gluon resummation, Phys. Rev. D 61 (2000) 014003 [Erratum ibid. D 64 (2001) 059903] [hep-ph/9906280]; Phenomenology of multiple parton radiation in semi-inclusive deep-inelastic scattering, Phys. Rev. D 64 (2001) 114011 hep-ph/0012261.

[29] A. Kulesza, G. Sterman and W. Vogelsang, Joint resummation in electroweak boson production, Phys. Rev. D 66 (2002) 014011 hep-ph/0202251.

[30] P.M. Nadolsky, Multiple parton radiation in hadroproduction at lepton hadron colliders, hep-ph/0108099.

[31] S. Catani, D. de Florian and M. Grazzini, Universality of non-leading logarithmic contributions in transverse momentum distributions, Nucl. Phys. B 596 (2001) 299 hep-ph/0008184.

[32] Y. Koike, J. Nagashima and W. Vogelsang, Resummation for polarized semi-inclusive deep-inelastic scattering at small transverse momentum, Nucl. Phys. B 744 (2006) 59 hep-ph/0602188.

[33] A. Weber, Soft gluon resummations for polarized Drell-Yan dimuon production, Nucl. Phys. B 382 (1992) 63.

[34] P.M. Nadolsky and C.P. Yuan, Soft parton radiation in polarized vector boson production: theoretical issues, Nucl. Phys. B 666 (2003) 3 hep-ph/0304001.

[35] J.C. Collins, Hard scattering in QCD with polarized beams, Nucl. Phys. B 394 (1993) 169 hep-ph/9207265.

[36] X.-D. Ji, J.-P. Ma and F. Yuan, QCD factorization for spin-dependent cross sections in DIS and Drell-Yan processes at low transverse momentum, Phys. Lett. B 597 (2004) 299 hep-ph/0405085.

[37] D.W. Sivers, Single spin production asymmetries from the hard scattering of point-like constituents, Phys. Rev. D 41 (1990) 83. 
[38] J.-W. Qiu and G. Sterman, Single transverse spin asymmetries, Phys. Rev. Lett. 67 (1991) 2264 .

[39] A. Idilbi, X.-D. Ji, J.-P. Ma and F. Yuan, Collins-Soper equation for the energy evolution of transverse-momentum and spin dependent parton distributions, Phys. Rev. D 70 (2004) 074021 hep-ph/0406302].

[40] A. Mendez, QCD predictions for semi-inclusive and inclusive leptoproduction, Nucl. Phys. B 145 (1978) 199.

[41] R. Meng, F.I. Olness and D.E. Soper, Semi-inclusive deeply inelastic scattering at small $q_{T}$, Phys. Rev. D 54 (1996) 1919 hep-ph/9511311.

[42] M. Diehl and S. Sapeta, On the analysis of lepton scattering on longitudinally or transversely polarized protons, Eur. Phys. J. C 41 (2005) 515 hep-ph/0503023.

[43] H. Eguchi, Y. Koike and K. Tanaka, Single transverse spin asymmetry for large-p $p_{T}$ pion production in semi-inclusive deep inelastic scattering, Nucl. Phys. B 752 (2006) 1 hep-ph/0604003.

[44] H. Eguchi, Y. Koike and K. Tanaka, Twist-3 formalism for single transverse spin asymmetry reexamined: semi-inclusive deep inelastic scattering, Nucl. Phys. B 763 (2007) 198 hep-ph/0610314.

[45] K. Hagiwara, K.-I. Hikasa and N. Kai, Time reversal odd asymmetry in semi-inclusive leptoproduction in quantum chromodynamics, Phys. Rev. D 27 (1983) 84.

[46] T. Gehrmann, Time-reversal-odd asymmetries at HERA, hep-ph/9608469; M. Ahmed and T. Gehrmann, Azimuthal asymmetries in hadronic final states at HERA, Phys. Lett. B 465 (1999) 297 hep-ph/9906503.

[47] A.V. Belitsky, X. Ji and F. Yuan, Final state interactions and gauge invariant parton distributions, Nucl. Phys. B 656 (2003) 165 hep-ph/0208038.

[48] C.J. Bomhof, P.J. Mulders and F. Pijlman, Gauge link structure in quark quark correlators in hard processes, Phys. Lett. B 596 (2004) 277 hep-ph/0406099.

[49] C.J. Bomhof, P.J. Mulders and F. Pijlman, The construction of gauge-links in arbitrary hard processes, Eur. Phys. J. C 47 (2006) 147 hep-ph/0601171.

[50] J.C. Collins and A. Metz, Universality of soft and collinear factors in hard-scattering factorization, Phys. Rev. Lett. 93 (2004) 252001 hep-ph/0408249].

[51] F. Hautmann, Endpoint singularities in unintegrated parton distributions, Phys. Lett. B 655 (2007) 26 hep-ph/0702196.

[52] I.O. Cherednikov and N.G. Stefanis, Renormalization, Wilson lines and transverse-momentum dependent parton distribution functions, Phys. Rev. D 77 (2008) 094001 arXiv:0710.1955.

[53] K. Goeke, A. Metz and M. Schlegel, Parameterization of the quark-quark correlator of a spin-1/2 hadron, Phys. Lett. B 618 (2005) 90 hep-ph/0504130.

[54] J.C. Collins, Leading-twist single-transverse-spin asymmetries: Drell-Yan and deep-inelastic scattering, Phys. Lett. B 536 (2002) 43 hep-ph/0204004.

[55] J.C. Collins and D.E. Soper, Parton distribution and decay functions, Nucl. Phys. B 194 (1982) 445. 
[56] D. Boer, Theoretical aspects of spin physics, hep-ph/0312149.

[57] A.A. Henneman, Scale dependence of correlations on the light-front, Ph.D. thesis, Vrije Universiteit, Amsterdam, The Netherlands (2005), http://www.nikhef.nl/pub/services/newbiblio/theses.php.

[58] D. Boer, Sudakov suppression in azimuthal spin asymmetries, Nucl. Phys. B 603 (2001) 195 hep-ph/0102071.

[59] M. Anselmino et al., The role of Cahn and Sivers effects in deep inelastic scattering, Phys. Rev. D 71 (2005) 074006 hep-ph/0501196].

[60] A. Méndez, A. Raychaudhuri and V.J. Stenger, QCD effects in semi-inclusive neutrino processes, Nucl. Phys. B 148 (1979) 499.

[61] A. König and P. Kroll, A realistic calculation of the azimuthal asymmetry in semi-inclusive deep inelastic scattering, Z. Physik C 16 (1982) 89.

[62] J.-G. Chay, S.D. Ellis and W.J. Stirling, Azimuthal asymmetry in lepton-photon scattering at high-energies, Phys. Rev. D 45 (1992) 46.

[63] K.A. Oganesian, H.R. Avakian, N. Bianchi and P. Di Nezza, Investigations of azimuthal asymmetry in semi-inclusive leptoproduction, Eur. Phys. J. C 5 (1998) 681 hep-ph/9709342.

[64] M. Anselmino, M. Boglione, A. Prokudin and C. Türk, Semi-inclusive deep inelastic scattering processes from small to large $p_{T}$, Eur. Phys. J. A31 (2007) 373 hep-ph/0606286.

[65] A.M. Kotzinian and P.J. Mulders, Longitudinal quark polarization in transversely polarized nucleons, Phys. Rev. D 54 (1996) 1229 hep-ph/9511420.

[66] J. Chay and S.M. Kim, Azimuthal correlation in lepton hadron scattering via charged weak-current processes, Phys. Rev. D 57 (1998) 224 hep-ph/9705284.

[67] J.C. Collins, Fragmentation of transversely polarized quarks probed in transverse momentum distributions, Nucl. Phys. B 396 (1993) 161 hep-ph/9208213.

[68] S. Meissner, A. Metz and K. Goeke, Relations between generalized and transverse momentum dependent parton distributions, Phys. Rev. D 76 (2007) 034002 [hep-ph/0703176].

[69] X. Artru and M. Mekhfi, Transversely polarized parton densities, their evolution and their measurement, Z. Physik C 45 (1990) 669.

[70] NA10 collaboration, S. Falciano et al., Angular distributions of muon pairs produced by $194 \mathrm{GeV} / \mathrm{c}$ negative pions, Z. Physik C 31 (1986) 513;

NA10 collaboration, M. Guanziroli et al., Angular distributions of muon pairs produced by negative pions on deuterium and tungsten, Z. Physik C 37 (1988) 545;

J.S. Conway et al., Experimental study of muon pairs produced by $252 \mathrm{GeV}$ pions on tungsten, Phys. Rev. D 39 (1989) 92,

FNAL-E866/NuSEA collaboration, L.Y. Zhu et al., Measurement of angular distributions of Drell-Yan dimuons in $p+d$ interaction at $800 \mathrm{GeV} / c$, Phys. Rev. Lett. 99 (2007) 082301 hep-ex/0609005.

[71] Belle collaboration, K. Abe et al., Measurement of azimuthal asymmetries in inclusive production of hadron pairs in $e^{+} e^{-}$annihilation at Belle, Phys. Rev. Lett. 96 (2006) 232002 hep-ex/0507063. 
[72] D. Boer, R. Jakob and P.J. Mulders, Asymmetries in polarized hadron production in $e^{+} e^{-}$ annihilation up to order $1 / Q$, Nucl. Phys. B 504 (1997) 345 hep-ph/9702281.

[73] D. Binosi and L. Theussl, JaxoDraw: a graphical user interface for drawing Feynman diagrams, Comput. Phys. Commun. 161 (2004) 76 hep-ph/0309015. 